\title{
ISOMORPHISM CONJECTURES IN ALGEBRAIC $K$-THEORY
}

\author{
F. T. FARRELL AND L. E. JONES
}

\section{INTRODUCTION}

In this paper we are concerned with the four functors $\mathscr{P}_{*}, \mathscr{P}^{\mathrm{diff}}, \mathscr{K}_{*}$, and $\mathscr{L}_{*}^{-\infty}$, which map from the category of topological spaces $X$ to the category of $\Omega$-spectra. The functor $\mathscr{P}_{*}\left(\right.$ ) (or $\left.\mathscr{P}^{\text {diff }}()\right)$ maps the space $X$ to the $\Omega$ spectrum of stable topological (or smooth) pseudoisotopies of $X$. (The spaces $\mathscr{P}_{i}(X), \mathscr{P}_{i}^{\text {diff }}(X), i \geq 0$, are Hatcher's deloopings of $\mathscr{P}_{0}(X), \mathscr{P}_{0}^{\text {diff }}(X)$ (cf. [29])). The functor $\mathscr{K}_{*}($ ) maps a path-connected space $X$ to the algebraic $K$ theoretic $\Omega$-spectrum for the integral group ring $\mathbf{Z} \pi_{1} X$. (The spaces $\mathscr{K}_{i}(X)$, $i \geq 0$, are the Gersten-Wagoner deloopings of $\mathscr{K}_{0}(X)$; cf. [27, 52].) $\mathscr{K}_{*}(X)$ is also defined on a nonpath-connected space $X$ to be $\bigoplus_{i \in I} \mathscr{K}_{*}\left(X_{i}\right)$, where $\left\{X_{i}: i \in I\right\}$ are the path components of $X$ and $\bigoplus_{i \in I} \mathscr{K}_{*}\left(X_{i}\right)$ indicates the direct limit of all finite products of the $\left\{\mathscr{K}_{*}\left(X_{i}\right): i \in I\right\}$. If $X$ is path connected then the functor $\mathscr{L}_{*}^{-\infty}()$ maps $X$ to the $L^{-\infty}$-surgery classifying spaces for oriented surgery problems with fundamental group $\pi_{1} X$ identified by their fourfold periodicity $\mathscr{L}_{k}^{-\infty}(X)=\mathscr{L}_{k+4}^{-\infty}(X)$ (cf. [35, 39, 44, 47]). In addition, if $X$ is not path connected then we set $\mathscr{L}_{*}^{-\infty}(X)=\bigoplus_{i \in I} \mathscr{L}_{*}^{-\infty}\left(X_{i}\right)$, where $\left\{X_{i}: i \in i\right\}$ are the path components of $X$.

Results obtained by the authors over the past five years (cf. [15-25] and, in particular, [19; 16, A.1, A.18]), together with many earlier results and conjectures which are reviewed in 1.6.1-1.6.6, suggest that, for any connected CWcomplex $X$ and for $\mathscr{S}_{*}()$ denoting any of the above functors, the $\Omega$-spectrum $\mathscr{S}_{*}(X)$ should be computable in a simple way from the values taken by $\mathscr{S}_{*}()$ on covering spaces of $X$, which have very simple fundamental groups. In more detail, we let $\mathscr{G}(X)$ denote the collection of all subgroups $H \subset \pi_{1} X$, which are either finite or virtually infinite cyclic. A group $H$ is virtually infinite cyclic if there is a short exact sequence of groups $1 \rightarrow \mathbf{Z} \rightarrow H \rightarrow F \rightarrow 1$ with $F$ equal to a finite group and $\mathbf{Z}$ equal to the infinite cyclic group. For each such group we let $X_{H} \rightarrow X$ denote the covering space projection corresponding to $H$. Then the $\Omega$-spectrum $\mathscr{S}_{*}(X)$ should be computable in a simple way from the $\Omega$-spectra $\left\{\mathscr{S}_{*}\left(X_{H}\right): H \in \mathscr{G}(X)\right\}$. The purpose of this paper is to formulate precisely a conjecture along these lines and to verify the conjecture for any $X$

Received by the editors June 5, 1991 and, in revised form, December 15, 1992.

1991 Mathematics Subject Classification. Primary 19D35, 19D50, 57N37, 57R67.

Both authors were supported in part by the National Science Foundation. 
whose fundamental group is a co-compact discrete subgroup of a virtually connected Lie group and when $\mathscr{S}_{*}()$ is either $\mathscr{P}_{*}()$ or $\mathscr{P}_{*}^{\text {diff }}()$. The conjecture will be verified for the same class of groups and for the functor $\mathscr{L}_{*}^{-\infty}()$ in a later publication. Based on these results we believe it is only a matter of time before these conjectures are verified when $\pi_{1} X$ is a discrete subgroup of a virtually connected Lie group. However, for more general classes of fundamental groups the authors regard these conjectures as only estimates which best fit the known data at this time. If these estimates prove incorrect for a larger class of groups, then one should look for the minimal class of subgroups $\mathscr{G}(X)$ of each $\pi_{1} X$ (this minimal class will now be larger than before), which makes the preceding conjectures true.

Here is an outline of the paper.

In $\S 1$ we give a precise formulation of our conjecture (cf. 1.6), which we outline as follows. Let $\Gamma$ denote a (discrete) group, and let $\mathscr{C}$ denote a class of subgroups of $\Gamma$. We define a universal $(\Gamma, \mathscr{C})$-space to be a regular cell complex $Z$ together with a group action $\Gamma \times Z \rightarrow Z$, which satisfies the following properties:

(a) For each $g \in \Gamma$ the homeomorphism $Z \rightarrow Z$ given by $z \rightarrow g(z)$ is cellular; moreover, for each cell $e \in Z$ if $g(e)=e$ then $g \mid e=$ inclusion.

(b) For any $z \in Z$ we have $\Gamma^{z} \in \mathscr{C}$, where $\Gamma^{z}$ is the isotropy group at $z$ for this action.

(c) For any $G \in \mathscr{C}$ the fixed point set of $G \times Z \rightarrow Z$ is a nonempty contractible subcomplex of $Z$.

For $X$ a connected CW-complex, choose a universal $\left(\pi_{1} X, \mathscr{G}(X)\right)$-space $\pi_{1} X \times$ $Z \rightarrow Z$ (their existence is proven in the appendix). Let $\widehat{X}$ denote the universal covering space for $X$, and let $\pi_{1} X \times(\hat{X} \times Z) \rightarrow \widehat{X} \times Z$ denote the diagonal action. We define $\rho: \mathscr{E}(X) \rightarrow \mathscr{B}(X)$ to be the quotient of the usual projection $\widehat{X} \times Z \rightarrow Z$ under the relevant $\pi_{1} X$-actions. Then $\rho$ is a "stratified fibration" (cf. 1.4, 1.5) whose fibers are homeomorphic to spaces in the collection $\left\{X_{H}: H \in \mathscr{G}(X)\right\}$. Our main conjecture (cf. 1.6) states that the $\Omega$-spectrum $\mathscr{S}_{*}(X)$ is homotopy equivalent to the "homology spectrum" for $\mathscr{B}(X)$ with respect to the system of twisted and stratified coefficients $\left\{\mathscr{S}_{*}\left(\rho^{-1}(b)\right): b \in \mathscr{B}(X)\right\}$ on $\mathscr{B}(X)$ (cf. 1.4).

We note that the truth of this conjecture for the functors $\mathscr{K}_{*}()$ and $\mathscr{L}_{*}^{-\infty}()$ would imply that the Novikov conjectures and the Borel conjecture are all true (cf. 1.6.2, 1.6.3). We note also that our present conjecture is equivalent to the conjecture made by the authors in $[22,4.1]$ for the special case that $X$ is aspherical with torsion-free fundamental group and $\mathscr{S}_{*}()=\mathscr{P}_{*}()$.

In $\S 2$ we formulate the main theorem of this paper (cf. 2.1 ), which states that the conjecture made in $\S 1$ is true for the functors $\mathscr{P}_{*}()$ and $\mathscr{P}^{\text {diff }}()$ on any space $X$ such that $\pi_{1} X$ is a co-compact discrete subgroup of a virtually connected Lie group. We state three propositions from which the theorem is then deduced. The first of these propositions is proven in the appendix, the second is proven in $\S 3$, and the third is proven in $\S 4$.

In $\S 3$ we prove the second of the propositions used in $\xi 2$. This proposition 
states that the conjecture of $\S 1$ is true for the functors $\mathscr{P}_{*}()$ and $\mathscr{P}_{*}^{\text {diff }}()$ on any space $X$ for which there is a properly discontinuous co-compact group action $\pi_{1} X \times M \rightarrow M$ by isometries of a simply connected symmetric Riemannian manifold $M$ having nonpositive sectional curvature everywhere. The arguments of this section are just a fibered and equivariant version of the arguments made by the authors in [24].

In $\S 4$ we prove the third of the propositions stated in $\S 2$. This proposition states that the conjecture of $\S 1$ is true for the functors $\mathscr{P}_{*}(), \mathscr{P}_{*}^{\text {diff }}()$ on any space $X$ for which $\pi_{1} X$ is a virtually poly-Z group. We use the first proposition stated in $\S 2$ together with geometric ideas from $[12,17]$ in an attempt to reduce the proof to an application of the second proposition stated in $\S 2$. We are able to reduce the proof of the third proposition stated in $\S 2$ to an application of Theorem 4.8, which is a variation on the theme of the second proposition in $\S 2$. The proof of Theorem 4.8, which combines the geometric ideas used in [12, $17,24]$, will be given in [23].

In the appendix we give an axiomatic treatment of the notion of a "universal $(\Gamma, \mathscr{C})$-space", which was used in $\S 1$ to formulate the main conjecture of this paper. We also verify several claims made in the main body of this paper (cf. 1.6.1, 1.6.2, 1.6.4) and give the proof for the first of the propositions stated and used in $\S 2$. That proposition is an induction result. It states that the fibered conjecture (cf. 1.7) of $\S 1$ is true for any of the functors $\mathscr{S}_{*}()$ described above on the total space of a fibration $p: X \rightarrow Y$ provided it is true for each of the following spaces: the space $Y$, and any space $Z$ such that $\pi_{1} Z$ is isomorphic to $p_{\#}^{-1}(H)$ for some group $H \in \mathscr{G}(Y)$. Here $X$ and $Y$ are connected $\mathrm{CW}$ complexes and $P_{\#}$ is an epimorphism.

\section{THE ISOMORPHISM CONJECTURES}

In this section we formulate our conjectures concerning the classical functors of algebraic and geometric topology $\mathscr{P}_{*}(), \mathscr{P}_{*}^{\text {diff }}(), \mathscr{K}_{*}(), \mathscr{L}_{*}^{-\infty}()$. We also discuss the relationship of these new conjectures to older conjectures (e.g., the Novikov conjectures).

1.1. The functors $\mathscr{P}_{*}(), \mathscr{P}_{*}^{\text {diff }}()$. If $X$ is a manifold and $i \geq 0$ an integer, then we denote by $P^{b}\left(X \times \mathbf{R}^{i}\right)$ the space of all topological pseudoisotopies $h: X \times \mathbf{R}^{i} \times[0,1] \rightarrow X \times \mathbf{R}^{i} \times[0,1]$, which are bounded in the $\mathbf{R}^{i}$-factor and have compact support in the $X$-factor. (Recall that $h$ is a topological pseudoisotopy if it is a homeomorphism such that $h \mid X \times \mathbf{R}^{i} \times 0=$ inclusion and that $h$ is bounded in the $\mathbf{R}^{i}$-factor if there is a number $\alpha>0$ such that for all $(x, y, t) \in X \times \mathbf{R}^{i} \times[0,1]$ we have that $\left|y-y^{\prime}\right| \leq \alpha$ holds where $h(x, y, t)=\left(x^{\prime}, y^{\prime}, t^{\prime}\right)$.) Note that if $I^{q}$ denotes the $q$-fold cartesian product of $[0,1]$ with itself, then there is an inclusion map

$$
P^{b}\left(X \times I^{q} \times \mathbf{R}^{i}\right) \rightarrow P^{b}\left(X \times I^{q+1} \times \mathbf{R}^{i}\right)
$$

(sometimes called "stabilization") obtained by forming the product of any topological pseudoisotopy $h: X \times I^{q} \times \mathbf{R}^{i} \times[0,1] \rightarrow X \times I^{q} \times \mathbf{R}^{i} \times[0,1]$ with the identity map $I \rightarrow I$. Define $\mathscr{P}_{i}(X)$ to be the direct limit space 
$\operatorname{limit}_{q \rightarrow \infty} P^{b}\left(X \times I^{q} \times \mathbf{R}^{i}\right)$, and for integers $i<0$ set $\mathscr{P}_{i}(X)=\Omega^{-i} \mathscr{P}_{0}(X)$. If $X$ is a smooth manifold, then by using only smooth pseudoisotopies $h: X \times$ $I^{q} \times \mathbf{R}^{i} \times[0,1] \rightarrow X \times I^{q} \times R^{i} \times[0,1]$ the preceding construction yields the spaces $\mathscr{P}_{i}^{\text {diff }}(X), i \in \mathbf{Z}$. We follow here the convention introduced in [29] of "rounding the corners" of the cylinder $X \times I^{q}$ before determining differentiable properties of the map $h$. Hatcher [29] has shown that $\mathscr{P}_{i}(X) \cong \Omega \mathscr{P}_{i+1}(X)$ and $\mathscr{P}_{i}^{\text {diff }}(X) \cong \Omega \mathscr{P}_{i+1}^{\text {diff }}(X)$ hold for all integers $i \geq 0$ (where $\cong$ means homotopy equivalent). Thus the collections $\mathscr{P}_{*}(X)=\left\{\mathscr{P}_{i}(X): i \in \mathbf{Z}\right\}$ and $\mathscr{P}_{*}^{\text {diff }}(X)=\left\{\mathscr{P}_{i}^{\text {diff }}(X): i \in \mathbf{Z}\right\}$ are $\Omega$-spectra. We note that both of the $\Omega$ spectra $\mathscr{P}_{*}(X)$ and $\mathscr{P}_{*}^{\text {diff }}(X)$ are the direct limits over all compact submanifolds $C \subset X$ of the $\Omega$-spectra $\left\{\mathscr{P}_{*}(C)\right\}$ and $\left\{\mathscr{P}_{*}^{\text {diff }}(C)\right\}$.

In the event that the topological space $X$ is not a manifold then for each integer $i \geq 0$ the space $\mathscr{P}_{i}(X)$ is a semisimplicial complex defined as follows. A typical $k$-simplex in $\mathscr{P}_{i}(X)$ consists of a compact smooth codimension zero submanifold $Y \subset \mathbf{R}^{n}$ of some Euclidean space $\mathbf{R}^{n}$, and mappings $h: \Delta^{k} \times Y \times$ $\mathbf{R}^{i} \times[0,1] \rightarrow \Delta^{k} \times Y \times \mathbf{R}^{i} \times[0,1]$ and $g: \Delta^{k} \times Y \rightarrow X$, such that for each point $p \in \Delta^{k}$, in the standard $k$-simplex $\Delta^{k}$, the restricted map $h: p \times Y \times$ $\mathbf{R}^{i} \times[0,1] \rightarrow p \times Y \times \mathbf{R}^{i} \times[0,1]$ is a topological pseudoisotopy bounded in the $\mathbf{R}^{i}$-factor (with bound independent of $p$ ). Two pairs of maps $h: \Delta^{k} \times Y \times \mathbf{R}^{i} \times$ $[0,1] \rightarrow \Delta^{k} \times Y \times \mathbf{R}^{i} \times[0,1], g: \Delta^{k} \times Y \rightarrow X$ and $h^{\prime}: \Delta^{k} \times Y^{\prime} \times \mathbf{R}^{i} \times[0,1] \rightarrow$ $\Delta^{k} \times Y^{\prime} \times \mathbf{R}^{i} \times[0,1], g^{\prime}: \Delta^{k} \times Y^{\prime} \rightarrow X$ represent the same simplex in $\mathscr{P}_{i}(X)$ if there is a codimension zero compact submanifold $A \subset Y$ such that the support of $h$ is contained in $\Delta^{k} \times A \times \mathbf{R}^{i} \times[0,1]$ and the equalities $Y^{\prime}=A \times I^{q}$, $h \times \mathrm{id}_{q} \mid \Delta^{k} \times A \times I^{q} \times \mathbf{R}^{i} \times[0,1]=h^{\prime}$, and $g \circ \pi=g^{\prime}$ all hold, where $\mathrm{id}_{q}: I^{q} \rightarrow I^{q}$ is the identity map and $\pi: \Delta^{k} \times Y \times I^{q} \rightarrow \Delta^{k} \times Y$ is the standard projection. By considering only smooth pseudoisotopies $h: \Delta^{k} \times Y \times \mathbf{R}^{i} \times[0,1] \rightarrow \Delta^{k} \times Y \times$ $\mathbf{R}^{i} \times[0,1]$ we obtain the spaces $\mathscr{P}_{i}^{\text {diff }}(X)$. Hatcher's arguments work in this semisimplicial setting also to show that the collections $\mathscr{P}_{*}(X)=\left\{\mathscr{P}_{i}(X): i \in \mathbf{Z}\right\}$ and $\mathscr{P}_{*}^{\text {diff }}(X)=\left\{\mathscr{P}_{i}^{\text {diff }}(X): i \in \mathbf{Z}\right\}$ are $\Omega$-spectra (where for integers $i<0$ we set $\mathscr{P}_{i}(X)=\Omega^{-i} \mathscr{P}_{0}(X)$ and $\left.\mathscr{P}_{i}^{\text {diff }}(X)=\Omega^{-i} \mathscr{P}_{0}^{\text {diff }}(X)\right)$. For further details the reader is referred to Hatcher [29] and Quinn [48].

1.2. The functor $\mathscr{K}_{*}()$. The value of the functor $\mathscr{K}_{*}()$ on the path-connected space $X$ is homotopy equivalent to the Gersten-Wagoner $\Omega$-spectrum for the algebraic $K$-theory of the integral group ring $\mathrm{Z} \pi_{1} X$. That is, $\mathscr{K}_{0}(X)$ is the usual space (defined by Quillen [46]) whose homotopy groups are equal to the algebraic $K$-groups $K_{j}\left(\mathbf{Z} \pi_{1} X\right), j \geq 0$, and for each integer $i \geq 0 \mathscr{K}_{i}(X)$ is the $i$-fold delooping of $\mathscr{K}_{0}(X)$ defined by Gersten [27] and Wagoner [52]. It is useful (e.g., see the definition of the assembly map in 1.4) to have a more functorial description of $\mathscr{K}_{*}(X)$, which is independent of the base point of $X$. Such a description appears in the work of Pederson and Weibel [40] and in the work of Quinn [50]. We note that $\mathscr{K}_{*}(X)$ is even defined when $X$ is not path connected by $\mathscr{K}_{*}(X)=\bigoplus_{i \in I} \mathscr{K}_{*}\left(X_{i}\right)$, where $\left\{X_{i}: i \in I\right\}$ are the 
path components of $X$ and $\bigoplus_{i \in I} \mathscr{K}_{*}\left(X_{i}\right)$ indicates the direct limit of all finite products of the $\left\{\mathscr{K}_{*}\left(X_{i}\right): i \in I\right\}$.

1.3. The functors $\mathscr{L}_{*}^{h}(), \mathscr{L}_{*}^{-\infty}()$. The functor $\mathscr{L}_{*}^{h}()$ is defined on any topological space $X$ which comes equipped with orientation data in the form of a map $w: X \rightarrow K\left(\mathbf{Z}_{2}, 1\right)$. For the duration of this paper we shall assume that $w$ is null-homotopic and shall therefore suppress it from our notation. For each integer $i$ the space $\mathscr{L}_{i}^{h}(X)$ is a semisimplicial space defined as follows. A typical $k$-simplex in $\mathscr{L}_{i}^{h}(X)$ consists of two oriented compact manifold 4tuples $\left(M, \partial_{-} M, \partial_{+} M, \Lambda M\right),\left(N, \partial_{-} N, \partial_{+} N, \Lambda N\right)$ and maps $g: N \rightarrow \Delta^{k}$, $h:\left(M, \partial_{-} M, \partial_{+} M, \Lambda M\right) \rightarrow\left(N, \partial_{-} N, \partial_{+} N, \Lambda N\right), f: N \rightarrow X$, all of which satisfy the following properties: $\operatorname{dim} M=k-i \bmod 4 ; \partial M=\partial_{-} M \cup \partial_{+} M$, where the $\partial_{\mp} M$ are compact codimension zero submanifolds of $\partial \bar{M}$ satisfying $\partial_{-} M \cap \partial_{+} M=\partial\left(\partial_{\mp} M\right)=\Lambda M$; the same properties hold for $\partial N, \partial_{\mp} N, \Lambda N$; $g^{-1}\left(\partial \Delta^{k}\right)=\partial_{-} N$, and $g: \partial_{-} N \rightarrow \partial \Delta^{k}, g \circ h: \partial_{-} M \rightarrow \partial \Delta^{k}$ are both in transverse position to all the simplices of $\partial \Delta^{k} ; h:(M, \partial M) \rightarrow(N, \partial N)$ is a degree one surgery normal map; and for each simplex $\Delta \in \Delta^{k}$ we have that the restricted map $h:(g \circ h)^{-1}(\Delta) \cap \partial_{+} M \rightarrow g^{-1}(\Delta) \cap \partial_{+} N$ is a homotopy equivalence. Any two 3-tuples of maps $(f, g, h),\left(f^{\prime}, g^{\prime}, h^{\prime}\right)$ as above represent the same $k$-simplex of $\mathscr{L}_{i}^{h}(X)$ if we have that $f^{\prime}=f \circ \pi, g^{\prime}=g \circ \pi$, and $h^{\prime}=h \times$ id, where id: $C P^{2} \rightarrow C P^{2}$ is the identity map of complex projective 2-space and $\pi: N \times C P^{2} \rightarrow N$ denotes the standard projection. Note that $\mathscr{L}_{i}^{h}(X)=\mathscr{L}_{i+4}^{h}(X)$. It can easily be seen (in the event that $X$ is path connected) that the space $\mathscr{L}_{i}^{h}(X)$ is homotopy equivalent to the $(4 n-i)$ th surgery classifying space for the fundamental group data $\pi_{1} w: \pi_{1} X \rightarrow \mathbf{Z}_{2}$ and for surgery up to homotopy equivalence, provided $4 n-i \geq 5$. Quinn [47] has shown that $\mathscr{L}_{i}^{h}(X) \cong \Omega_{\mathscr{L}_{i+1}^{h}}(X)$ holds for all $i \in \mathbf{Z}$. Thus the collection of spaces $\mathscr{L}_{*}^{h}(X)=\left\{\mathscr{L}_{i}^{h}(X): i \in \mathbf{Z}\right\}$ is an $\Omega$-spectrum, which exhibits 4-fold periodicity.

Unfortunately the functor $\mathscr{L}_{*}^{h}()$ is not the one for which the conjecture given below can be verified in many cases. This is the reason for introducing the more complicated functor $\mathscr{L}_{*}^{-\infty}$ ( ), which can be constructed from $\mathscr{L}_{*}^{h}($ ) as follows. Set $L_{*}^{-\infty}(X)$ equal to the direct limit of the sequence of $\Omega$-spectra

$$
\mathscr{L}_{*}^{h}(X) \rightarrow \mathscr{L}_{*-1}^{h}\left(X \times T^{1}\right) \rightarrow \mathscr{L}_{*-2}^{h}\left(X \times T^{2}\right) \rightarrow \mathscr{L}_{*-3}^{h}\left(X \times T^{3}\right) \rightarrow \cdots,
$$

where $T^{n}$ denotes the $n$-dimensional torus and the map $\mathscr{L}_{*-n}^{h}\left(X \times T^{n}\right) \rightarrow$ $\mathscr{L}_{*-n-1}^{h}\left(X \times T^{n+1}\right)$ is obtained by forming the Cartesian product of each simplex of $\mathscr{L}_{*-n}^{h}\left(X \times T^{n}\right)$ with the circle $S^{1}$. Now we can define $\mathscr{L}_{*}^{-\infty}(X)$ to be the direct limit of the sequence of $\Omega$-spectra

$$
L_{*}^{-\infty}(X) \stackrel{r_{*}}{\rightarrow} L_{*}^{-\infty}(X) \stackrel{r_{*}}{\rightarrow} L_{*}^{-\infty}(X) \stackrel{r_{*}}{\rightarrow} \cdots,
$$

where $r_{*}: L_{*}^{\infty}(X) \rightarrow L_{*}^{\infty}(X)$ is a map of $\Omega$-spectra defined as follows. Let 
the maps $h:\left(M, \partial_{-} M, \partial_{+} M, \Lambda M\right) \rightarrow\left(N, \partial_{-} N, \partial_{+} N, \Lambda N\right), g: N \rightarrow \Delta^{k}$, $f: N \rightarrow X \times T^{n}$ represent a $k$-simplex in the space $\mathscr{L}_{*-n}^{h}\left(X \times T^{n}\right)$. We may assume that each restricted map $f \mid g^{-1}(\Delta), \Delta \in \Delta^{k}$, is in transverse position to each manifold in the sequence $X \times 0 \subset X \times T^{1} \subset X \times T^{2} \subset \cdots \subset X \times T^{n}$ (where 0 is the origin in $T^{n}$ and $T^{1} \subset T^{2} \subset \cdots \subset T^{n}$ is the usual filtration of $T^{n}$ by smaller tori). We would like to put each restricted map $h \mid(g \circ h)^{-1}(\Delta), \Delta \in$ $\Delta^{k}$, in transverse position to each manifold in the sequence $f^{-1}(X \times 0) \subset$ $f^{-1}\left(X \times T^{1}\right) \subset f^{-1}\left(X \times T^{2}\right) \subset \cdots \subset f^{-1}\left(X \times T^{n}\right)$ in such a way that for each simplex $\Delta \in \Delta^{k}$ and each integer $0 \leq i \leq n$ we have that the restricted map $h: \partial_{+} M \cap(g \circ h)^{-1}(\Delta) \cap(f \circ h)^{-1}\left(X \times T^{i}\right) \rightarrow \partial_{+} N \cap g^{-1}(\Delta) \cap f^{-1}\left(X \times T^{i}\right)$ is a homotopy equivalence (here $T^{0}=0$ ). The problem of doing this is known as a "splitting problem" to the experts in surgery theory and cannot always be solved; however, this splitting problem can always be solved if we first form the cartesian product of the $k$-simplex represented by the 3-tuple $(f, g, h)$ with a torus $T^{q}$ of sufficiently large enough dimension-thereby forming a $k$-simplex in $\mathscr{L}_{*-n-q}^{h}\left(X \times T^{n+q}\right)$, which is represented by a 3-tuple of maps $(\hat{f}, \hat{g}, \hat{h})-(\mathrm{cf}$. Farrell and Hsiang [11] and Farrell and Jones [16, $\S 9])$. Let the 3-tuple of maps $\left(f^{\prime}, g^{\prime}, h^{\prime}\right)$ be defined by $f^{\prime}=\hat{f}\left|\hat{f}^{-1}\left(X \times 0 \times T^{q}\right), g^{\prime}=\hat{g}\right| \hat{f}^{-1}\left(X \times 0 \times T^{q}\right)$, $h^{\prime}=\hat{h} \mid(\hat{f} \circ \hat{h})^{-1}\left(X \times 0 \times T^{q}\right)$, where 0 denotes the origin in $T^{n}$ and we are using the factorization $T^{n+q}=T^{n} \times T^{q}$. Now we set $r_{*}(f, g, h)=\left(f^{\prime}, g^{\prime}, h^{\prime}\right)$. We remark that if $X$ is path connected then $\mathscr{L}_{i}^{-\infty}(X)$ is homotopy equivalent to the surgery classifying space, which has for its $k$ th homotopy group the surgery group $L_{k-i}^{-\infty}\left(\pi_{1} X\right)$ introduced by Ranicki [43]. We note also that there is a natural "inclusion" map $\mathscr{L}_{*}^{h}(X) \rightarrow \mathscr{L}_{*}^{-\infty}(X)$ of $\Omega$-spectra, which induces (after tensoring with $\mathbf{Z}\left[\frac{1}{2}\right]$ ) an isomorphism on the homotopy groups.

For more details about surgery classifying spaces the reader is referred to [16, $\S 9,35,39,44,47,55]$.

1.4. Homology theory with coefficients in stratified and twisted $\Omega$-spectra and the assembly map. Generalized homology theories, where the $\Omega$-spectrum of coefficients $\mathscr{S}_{*}$ is "stratified and twisted" over a space $X$, have been discussed in [34, 48, 49]. The following version is taken from Quinn's paper [48].

A mapping $f: E \rightarrow X$ is a simplicially stratified fibration if there is a triangulation $K$ for the space $X$ such that the following hold.

1.4.1. (a) For each simplex $\Delta \in K$ the mapping $f: f^{-1}(\Delta-\partial \Delta) \rightarrow \Delta-\partial \Delta$ is a Serre fibration.

(b) For each simplex $\Delta \in K$ there is collaring $c: \partial \Delta \times[0,1] \rightarrow \Delta$ for $\partial \Delta$ in $\Delta$ with $c(\partial \Delta \times 0)=\partial \Delta$; we set $U=\operatorname{Image}(c)$. There are also deformation retracts $r: U \times[0,1] \rightarrow U$ of $U$ onto $\partial \Delta$ and $r^{\prime}: f^{-1}(U) \times[0,1] \rightarrow f^{-1}(U)$ of $f^{-1}(U)$ onto $f^{-1}(\partial \Delta)$, such that $f\left(r^{\prime}(p, t)\right)=r(f(p), t)$ and $r(c(x, s), t)=$ $c(x,(1-t) s)$ hold for all $p \in f^{-1}(U), x \in \partial \Delta$, and all $s, t \in[0,1]$.

Let $K^{(1)}$ denote the first barycentric subdivision of the triangulation $K$ of 1.4.1, and let $\mathscr{S}_{*}()$ denote any one of the functors described in 
1.1-1.3. For each integer $j$ define the space $\mathscr{S}_{j}(f)$ to be the quotient space $\left(\bigcup_{\Delta \in K^{(1)}} \mathscr{S}_{j}\left(f^{-1}(\Delta)\right) \times \Delta\right) / \sim$, where the equivalence relation $\sim$ simply identifies $\mathscr{S}_{j}\left(f^{-1}\left(\Delta^{\prime}\right)\right) \times \Delta^{\prime}$ with its image in $\mathscr{S}_{j}\left(f^{-1}(\Delta)\right) \times \Delta$ under the map induced by the inclusion $\Delta^{\prime} \subset \Delta$, for every pair $\Delta^{\prime}, \Delta \in K^{(1)}$ satisfying $\Delta^{\prime} \subset \Delta$. Note that $X$ may be identified with the subspace $\left(\bigcup_{\Delta \in K^{(1)}} I_{\Delta} \times \Delta\right) / \sim$ of $\mathscr{S}_{j}(f)$, where $I_{\Delta}$ is the base point of the space $\mathscr{S}_{j}\left(f^{-1}(\Delta)\right)$. (The base points of the spaces $\mathscr{P}_{j}\left(f^{-1}(\Delta)\right), \mathscr{P}_{j}^{\text {diff }}\left(f^{-1}(\Delta)\right), j \geq 0$, are the identity pseudoisotopies; the base points of the spaces $\mathscr{L}_{j}^{-\infty}\left(f^{-1}(\Delta)\right), j \in \mathbf{Z}$, are the empty vertices, i.e., the surgery normal maps with empty domain and range.) The collection of spaces $\mathscr{S}_{*}(f)=\left\{\mathscr{S}_{j}(f): j \in \mathbf{Z}\right\}$ is not in general an $\Omega$-spectrum; however, $\mathscr{S}_{*}(f)$ is an exspectrum, cf. [48, Appendix].

The homology spectrum $\mathbb{H}_{*}\left(X, \mathscr{S}_{*}(f)\right)$ for $X$ with (stratified and twisted) coefficients in $\mathscr{S}_{*}(f)$ is the collection of spaces $\left\{\mathbb{H}_{i}\left(X, \mathscr{S}_{*}(f)\right): i \in \mathbf{Z}\right\}$ defined by

$$
\mathbb{H}_{i}\left(X, \mathscr{S}_{*}(f)\right)=\operatorname{limit}_{j \rightarrow \infty} \Omega^{j}\left(\mathscr{S}_{j+i}(f) / X\right) .
$$

Note that the homology spectrum $\mathbb{H}_{*}\left(X, \mathscr{S}_{*}(f)\right)$ is an $\Omega$-spectrum. The kth homology group $H_{k}\left(X, \mathscr{S}_{*}(f)\right)$ for $X$ with (stratified and twisted) coefficients in $\mathscr{S}_{*}(f)$ is defined to be the $k$ th homotopy group of the $\Omega$-spectrum $\mathbb{H}_{*}\left(X, \mathscr{S}_{*}(f)\right)$. We note that if $f: E \rightarrow X$ is a trivial fiber bundle with fiber equal the space $Y$ then we have that $\mathbb{H}_{*}\left(X, \mathscr{S}_{*}(f)\right)=\mathbb{H}_{*}\left(X, \mathscr{S}_{*}(Y)\right)$ and $H_{k}\left(X, \mathscr{S}_{*}(f)\right)=H_{k}\left(X, \mathscr{S}_{*}(Y)\right)$ for all $k^{*} \in \mathbf{Z}$, where $\mathbb{H}_{*}\left(X, \mathscr{S}_{*}(Y)\right)$ is just the usual homology spectrum for $X$ with (unstratified and untwisted) coefficients in the $\Omega$-spectrum $\mathscr{S}_{*}(Y)$ and $H_{k}\left(X, \mathscr{S}_{*}(Y)\right), k \in \mathbf{Z}$, are just the usual generalized homology groups for $X$ with (unstratified and untwisted) coefficients in the $\Omega$-spectrum $\mathscr{S}_{*}(Y)$.

The homology spectrum $\mathbb{H}_{*}^{*}\left(X, \mathscr{S}_{*}(f)\right)$ can be constructed for any map $f: E \rightarrow X$ whose range space can be triangulated. However, the following lemma holds only for maps $f: E \rightarrow X$ which satisfy 1.4.1 (cf. [48, Appendix]).

1.4.2. Lemma. Let $f: E \rightarrow X$ be a simplicially stratified fibration as in 1.4.1. Then the following hold.

(a) The homology spectrum $\mathbb{H}_{*}\left(X, \mathscr{S}_{*}(f)\right)$ is independent of the triangulation $K$ satisfying 1.4.1(a), (b).

(b) There is a spectral sequence with $E_{i, j}^{2}=H_{i}\left(X, \pi_{j} \mathscr{S}_{*}(f)\right)$, which abuts to $H_{i+j}\left(X, \mathscr{S}_{*}(f)\right)$. Here $\pi_{j} \mathscr{S}_{*}(f)$ denotes the stratified system of groups $\left\{\pi_{j} \mathscr{S}_{*}\left(f^{-1}(x)\right): x \in X\right\}$ over $X$, where $\pi_{j} \mathscr{S}_{*}\left(f^{-1}(x)\right)$ is the jth homotopy group of the $\Omega$-spectrum $\mathscr{S}_{*}\left(f^{-1}(x)\right)$.

1.4.2.1. Remark. Any simplicially stratified fibration $f: E \rightarrow X$ as in 1.4.1(a), (b) which has a finite-dimensional base space $X$, is also a "stratified system of fibrations" in the sense of $[48,8.2]$. Thus we may apply $[48,8.4]$ to get 1.4.2(a). If the base space $X$ is compact then we may apply [48, 8.7] to get 1.4.2(b). To prove 1.4.2(a), (b) without these restrictions on the base space $X$. we note that $\mathbb{H}_{*}\left(X, \mathscr{S}_{*}(f)\right)$ has been defined in $[48$, p. 420] independently of 
any triangulation of $X$ in such a way that $\mathbb{H}_{*}\left(X, \mathscr{P}_{*}(f)\right)$ is equal to the direct limit of the $\Omega$-spectra $\left\{\mathbb{H}_{*}\left(C, \mathscr{P}_{*}\left(f \mid f^{-1}(C)\right)\right): C=\right.$ compact subset of $\left.X\right\}$. We also note that our triangulation-dependent definition for $\mathbb{H}_{*}\left(X, \mathscr{S}_{*}(f)\right)$ given above enjoys the property that $\mathbb{H}_{*}\left(X, \mathscr{S}_{*}(f)\right)$ is the direct limit of the $\Omega$-spectra $\left\{\mathbb{H}_{*}\left(L, \mathscr{S}_{*}(f \mid L): L=\right.\right.$ finite subcomplex of $\left.K\right\}$, where $K$ is a triangulation of $X$ as in 1.4.1(a), (b). Now a direct limit argument (where the direct limit is taken over compact subsets of $X$ ) shows that 1.4.2(a), (b) for general $X$ follow from the special cases of 1.4.2(a), (b) for $X$ compact.

If $f: E \rightarrow X$ and $f^{\prime}: E^{\prime} \rightarrow X^{\prime}$ are two simplicially stratified fibrations then by a fibered homotopy equivalence from $f$ to $f^{\prime}$ we mean a map $h: E \rightarrow E^{\prime}$ for which there exists three other maps, namely, $h^{\prime}: E^{\prime} \rightarrow E, H: E \times[0,1] \rightarrow E$, and $H^{\prime}: E^{\prime} \times[0,1] \rightarrow E^{\prime}$ satisfying the following properties: $h$ and $h^{\prime}$ preserve fibers; $H$ is a homotopy from the identity map on $E$ to the composite map $h^{\prime} \circ h$, which preserves the fibers of $f$; and $H^{\prime}$ is a homotopy from the identity map on $E^{\prime}$ to the composite map $h \circ h^{\prime}$, which preserves the fibers of $f^{\prime}$. We note that any fibered homotopy equivalence $h: E \rightarrow E^{\prime}$ induces an equivalence $\mathbb{H}_{*}\left(X, \mathscr{S}_{*}(f)\right) \cong \mathbb{H}_{*}\left(X^{\prime}, \mathscr{S}_{*}\left(f^{\prime}\right)\right)$.

The inclusion maps $\left\{f^{-1}(\Delta) \subset E: \Delta \in K^{(1)}\right\}$ induce maps $\mathscr{S}_{j}\left(f^{-1}(\Delta)\right)$ $\rightarrow \mathscr{S}_{j}(E)$, which in turn induce maps $\phi_{j}: \mathscr{S}_{j}(f) \rightarrow \mathscr{S}_{j}(E)$. Define $A_{j}$ : $\mathbb{H}_{j}\left(X, \mathscr{S}_{*}(f)\right) \rightarrow \mathscr{S}_{j}(E)$ to be the direct limit as $i \rightarrow \infty$ of the composite maps

$$
\Omega^{i}\left(\mathscr{S}_{j+i}(f) / X\right) \stackrel{\Omega^{i}\left(\phi_{j+i}\right)}{\longrightarrow} \Omega^{i}\left(\mathscr{S}_{j+i}(E)\right) \cong \mathscr{S}_{j}(E)
$$

Note that the collection of all such maps, denoted by $A_{*}: \mathbb{H}_{*}\left(X, \mathscr{S}_{*}(f)\right) \rightarrow$ $\mathscr{S}_{*}(E)$, is a mapping of $\Omega$-spectra, which is called the assembly map. In the special case when $f: E \rightarrow X$ is the trivial fiber bundle with fiber equal $Y$, then we have an assembly map $A_{*}: \mathbb{H}_{*}\left(X, \mathscr{S}_{*}(Y)\right) \rightarrow \mathscr{S}_{*}(E)$, which coincides with the usual assembly map $A_{*}: \mathbb{H}_{*}\left(X, \mathscr{S}_{*}(\mathrm{pt})\right) \rightarrow \mathscr{S}_{*}(X)$ if $Y=$ point. The assembly map can be defined for any map $f: E \rightarrow X$ whose range can be triangulated. However, the following lemma holds only for those $f: E \rightarrow X$ which satisfy 1.4.1 (cf. [48, Appendix] and Remark 1.4.2.1).

1.4.3. Lemma. If $f: E \rightarrow X$ is a simplicially stratified fibration as in 1.4.1 then the assembly map $A_{*}: \mathbb{H}_{*}\left(X, \mathscr{S}_{*}(f)\right) \rightarrow \mathscr{S}_{*}(E)$ is independent of the triangulation $K$ satisfying properties (a) and (b) of 1.4.1.

1.5. The simplicially stratified fibration $\rho: \mathscr{E}(X) \rightarrow \mathscr{B}(X)$. Let $\Gamma$ denote a (discrete) group, and let $\mathscr{C}$ denote a collection of subgroups of $\Gamma$. We gave the definition of a "universal $(\Gamma, \mathscr{C})$-space" in the outline of $\S 1$ in the introduction. The class of subgroups $\mathscr{C}$ is full if and only if it is closed with respect to the operations of taking subgroups and taking conjugates of groups. In the appendix of this paper we give an axiomatic treatment of universal $(\Gamma, \mathscr{C})$-spaces for full classes of subgroups $\mathscr{C}$. In particular, we prove that for any full class of subgroups $\mathscr{C}$ of $\Gamma$ there exists a universal $(\Gamma, \mathscr{C})$-space, which is unique up to $\Gamma$-equivariant homotopy equivalence (cf. Theorems A.2, A.3).

Let $X$ denote any connected CW-complex, and let $\mathscr{G}(X)$ denote the class of all subgroups $H \subset \pi_{1} X$ which are either finite or for which there is a short 
exact sequence of groups $1 \rightarrow \mathbf{Z} \rightarrow H \rightarrow F \rightarrow 1$ with $F$ a finite group and $\mathbf{Z}$ the infinite cyclic group. (Stated more concisely, $\mathscr{G}(X)$ consists of all virtually cyclic subgroups of $\pi_{1} X$.) Note that $\mathscr{G}(X)$ is a full class of subgroups of $\pi_{1} X$. So, by the result just quoted, there is a universal $\left(\pi_{1} X, \mathscr{G}(X)\right)$-space $\pi_{1} X \times A \rightarrow A$. Let $\widehat{X}$ denote the universal covering space for $X$, and let $\pi_{1} X \times(\widehat{X} \times A) \rightarrow \widehat{X} \times A$ denote the diagonal action. Define $\rho: \mathscr{E}(X) \rightarrow \mathscr{B}(X)$ to be the quotient of the standard projection $\widehat{X} \times A \rightarrow A$ under the relevant $\pi_{1} X$-actions, and define $f: \mathscr{E}(X) \rightarrow X$ to be the quotient of the standard projection $\widehat{X} \times A \rightarrow \widehat{X}$ under the relevant $\pi_{1}(X)$-actions. Because the universal $\left(\pi_{1} X, \mathscr{G}(X)\right)$-space $\pi_{1} X \times A \rightarrow A$ is uniquely determined by $X$ up to $\pi_{1} X$ equivariant homotopy type, we get the following lemma. Recall that for each $H \in \mathscr{G}(X)$ we denote by $X_{H} \rightarrow X$ the covering space for $X$ corresponding to $H$.

1.5.1. Lemma. $\rho: \mathscr{E}(X) \rightarrow \mathscr{B}(X)$ is a simplicially stratified fibration. Each fiber $\rho^{-1}(z)$ of $\rho$ is one of the connected covering spaces $\left\{X_{H}: H \in \mathscr{G}(X)\right\}$ for $X$, in fact, the restricted map $f: \rho^{-1}(z) \rightarrow X$ is a covering space projection whose image on the fundamental group level is contained in $\mathscr{G}(X)$. Moreover, $\rho: \mathscr{E}(X) \rightarrow \mathscr{B}(X)$ is uniquely determined up to fibered homotopy type by the homotopy type of $X$.

1.6. The isomorphism conjecture. Let $\mathscr{S}_{*}($ ) denote any of the $\Omega$-spectravalued functors of $1.1-1.3$, and let $X$ denote any connected $\mathrm{CW}$-complex. We conjecture that the composite map

$$
\mathbb{H}_{*}\left(\mathscr{B}(X), \mathscr{S}_{*}(\rho)\right) \stackrel{\mathscr{S}_{*}(f) \circ A_{*}}{\longrightarrow} \mathscr{S}_{*}(X)
$$

is an equivalence of $\Omega$-spectra, where $A_{*}$ is the assembly map for the simplicially stratified fibration $\rho: \mathscr{E}(X) \rightarrow \mathscr{B}\left(X^{*}\right)$ and $\mathscr{S}_{*}(f)$ is the image of the map $f: \mathscr{E}(X) \rightarrow X$ under the functor $\mathscr{S}_{*}()$.

1.6.0.1. Convention. In Conjecture 1.6, and in the rest of this paper, we adopt the following convention: each $\Omega$-spectrum $\mathscr{S}_{*}$ is automatically replaced by the $\Omega$-spectrum $S \mathscr{S}_{*}$, where $S \mathscr{S}_{i}$ is the topological realization of the semisimplicial complex of singular simplices in $\mathscr{S}_{i}$, and any map $f_{*}: \mathscr{S}_{*} \rightarrow \mathscr{T}_{*}$ between $\Omega$-spectra is automatically replaced by the map $S f_{*}: S \mathscr{S}_{*} \rightarrow S \mathscr{T}_{*}^{*}$, which is induced by $f_{*}$. As a result of this convention, any weak equivalence between $\Omega$-spectra $f_{*}^{*}: \mathscr{S}_{*} \rightarrow \mathscr{T}_{*}$ (i.e., each $f_{i}$ induces an isomorphism on homotopy groups) is an equivalence of $\Omega$-spectra (i.e., each $f_{i}$ is a homotopy equivalence).

1.6.0.2. Remark. Note that Conjecture 1.6 can also be formulated for the functors $\mathscr{L}_{*}^{h}()$ and $\mathscr{L}_{*}^{s}()$; for these functors 1.6 states that each of the maps

$$
\mathbb{H}_{*}\left(\mathscr{B}(X), \mathscr{L}_{*}^{h}(\rho)\right) \stackrel{\mathscr{L}_{*}^{h}(f) \circ A_{*}^{h}}{\longrightarrow} \mathscr{L}_{*}^{h}(X)
$$

and

$$
\mathbb{H}_{*}\left(\mathscr{B}(X), \mathscr{L}_{*}^{s}(\rho)\right) \stackrel{\mathscr{L}_{*}^{s}(f) \circ A_{*}^{s}}{\longrightarrow} \mathscr{L}_{*}^{s}(X)
$$


is an equivalence of $\Omega$-spectra. We can verify 1.6 for many spaces $X$ for the functor $\mathscr{L}_{*}^{-\infty}()$; however, for these same spaces our verifications often break down when applied to the functor $\mathscr{L}_{*}^{h}()$, or to the functor $\mathscr{L}_{*}^{s}()$, if $\pi_{1} X$ contains elements of finite order. Therefore, at this time we do not know if 1.6 is a reasonable conjecture to make for the functors $\mathscr{L}_{*}^{h}(), \mathscr{L}_{*}^{s}()$ when $\pi_{1} X$ contains elements of finite order. It seems that 1.6 is a reasonable conjecture for these functors if $\pi_{1} X$ is torsionfree (cf. 1.6.1). For general $X$ is it possible that the truth of Conjecture 1.6 for $\mathscr{L}_{*}^{h}\left(\right.$ ) (or for $\mathscr{L}_{*}^{s}()$ ) is just a formal consequence of the truth of Conjecture 1.6 for both of the functors $\mathscr{K}_{*}()$ and $\mathscr{L}_{*}^{-\infty}() ?$

In the following remarks we consider some of the consequences of the truth of Conjecture 1.6 for each of the $\Omega$-spectra-valued functors of 1.1-1.3.

1.6.1. $\pi_{1}(X)$ is torsionfree and $X$ is aspherical. In this case each $H \in \mathscr{G}(X)$ is either the infinite cyclic group or the trivial group. Let $\pi_{1} X \times A \rightarrow A$ denote a universal $\left(\pi_{1} X, \mathscr{G}(X)\right)$-space. Note, by Theorem A.2 of the appendix, that there is a $\pi_{1} X$-equivariant map $g: \widehat{X} \rightarrow A$, which is unique up to equivariant homotopy. Note that the quotient of the map $1_{\widehat{X}} \times g: \widehat{X} \rightarrow \widehat{X} \times A$ under the relevant $\pi_{1} X$-actions is a fiber preserving map between the simplicially stratified fibrations $1_{X}: X \rightarrow X$ and $\rho: \mathscr{E}(X) \rightarrow \mathscr{B}(X)$, which we denote by $I$. The mapping $I$ induces a map between $\Omega$-spectra $I_{*}: \mathbb{H}_{*}\left(X, \mathscr{S}_{*}(\mathrm{pt})\right) \rightarrow$ $\mathbb{H}_{*}\left(\mathscr{B}(X), \mathscr{S}_{*}(\rho)\right)$, which when composed with the assembly map $A_{*}$ : $\mathbb{H}_{*}\left(\mathscr{B}(X), \mathscr{S}_{*}(\rho)\right) \rightarrow \mathscr{S}^{*}(\mathscr{E}(X))$ and with the map $\mathscr{S}_{*}(f): \mathscr{S}_{*}(\mathscr{E}(X)) \rightarrow$ $\mathscr{\mathscr { S }}_{*}^{*}(X)$, yields the usual assembly map $\mathbb{H}_{*}\left(X, \mathscr{S}_{*}(\mathrm{pt})\right) \rightarrow \mathscr{\mathscr { S }}_{*}^{*}(X)$.

For $\mathscr{S}_{*}()=\mathscr{K}_{*}(), \mathscr{L}_{*}^{\beta}()$, where $\beta=-\infty, h, s$, the map $I_{*}$ is an equivalence of $\Omega$-spectra. (This last fact will be proven in Remark A.11 of the appendix.) So 1.6 reduces (for $\mathscr{S}_{*}()=\mathscr{K}_{*}(), \mathscr{L}_{*}^{\beta}()$ ) to the conjecture that the usual assembly map

(a) $\mathbb{H}_{*}\left(X, \mathscr{S}_{*}(\mathrm{pt})\right) \rightarrow \mathscr{S}_{*}(X)$ is an equivalence.

We note that (a) has been verified when $\pi_{1} X$ is a virtually poly-Z group (cf. $[17,50])$. It has also been verified for $\mathscr{S}_{*}()=\mathscr{L}_{*}^{\beta}()$, for $\beta=-\infty, h, s$, when either $X$ is a compact Riemannian manifold with sectional curvature $K \leq 0$ everywhere (cf. $[21,25]$ ) or when $X$ is a complete Riemannian manifold with $K=-1$ everywhere (cf. [16]).

In the case that $\mathscr{S}_{*}()=\mathscr{K}_{*}()$ it is known that the assembly map in (a) is an equivalence of $\Omega$-spectra if and only if we have the following calculations (cf. $[27,50,53])$ :

(b) $W h_{i}\left(\pi_{1} X\right)=0$ for ail integers $i \geq 1$.

(c) $\tilde{K}_{0}\left(\mathbf{Z} \pi_{1} X\right)=0$ and $K_{i}\left(\mathbf{Z} \pi_{1} X\right)=0$ for all integers $i<0$.

That (a), (b), and/or (c) might be true for $\pi_{1} X$ equal to an arbitrary torsionfree group is a possibility that has been noticed by many experts (cf. [30, 31, $38,51,55]$ ); whereas Conjecture 1.6 states a new possibility.

If $X$ is a compact aspherical manifold and $\mathscr{S}_{*}()=\mathscr{L}_{*}^{h}()$ then it is well known that property (a) and the equality $W h\left(\pi_{1} X\right)^{*}=0$ are together equivalent to the generalized Borel conjecture in dimensions $\geq 5$ (cf. 1.6.3). 
If $\mathscr{S}_{*}()=\mathscr{P}_{*}(\mathrm{l}$ then Conjecture 1.6 can be seen to be equivalent to another conjecture made by the authors in $[22,4.1]$. This conjecture has been verified for $X$ equal to any compact Riemannian manifold having sectional curvature $K \leq 0$ everywhere (cf. [22, 24]).

Finally we mention that Bizhong $\mathrm{Hu}$ [33a] has verified $W h\left(\pi_{1} M\right)=0$ for any finite simplicial complex $M$ with sectional curvature $K \leq 0$. Hu's result implies $W h(\Gamma)=0$ for $\Gamma$ any cocompact discrete subgroup of $\operatorname{SL}\left(n, Q_{p}\right)$ where $Q_{p}$ denotes the $p$-adic numbers. For this same class of fundamental groups $\mathrm{Hu}[33 \mathrm{~b}]$ can verify (a) for surgery theory.

1.6.2. The Novikov conjectures. We remark that in order to prove Conjecture 1.6 for the functors $\mathscr{K}_{*}()$ and $\mathscr{L}_{*}^{-\infty}()$ it suffices to verify 1.6 when $X$ is an aspherical space. The same is true of the $K$-theoretic and $L$-theoretic Novikov conjectures, which we recall for the reader now. The $L$-theoretic Novikov conjecture (Novikov's original conjecture) is equivalent to (a) below, and the $K$-theoretic Novikov conjecture (due to W.-c. Hsiang [30, 31]) is formulated in (b) below.

(a) For any aspherical CW-complex $X$ the usual assembly map

$$
\mathbb{H}_{*}\left(X, \mathscr{L}_{*}^{-\infty}(\mathrm{pt})\right) \rightarrow \mathscr{L}_{*}^{-\infty}(X)
$$

is rationally injective on the homotopy group level.

(b) For any aspherical CW-complex $X$ the usual assembly map

$$
\mathbb{H}_{*}\left(X, \mathscr{K}_{*}(\mathrm{pt})\right) \rightarrow \mathscr{K}_{*}(X)
$$

is rationally injective on the homotopy group level.

The truth of Conjecture 1.6 implies that both of the Novikov conjectures are true. This is seen by showing that the map

$$
\begin{array}{rcc}
X & \rightarrow & \mathscr{E}(X) \\
1_{X} \downarrow & I & \downarrow \rho \\
X & \rightarrow & \mathscr{B}(X)
\end{array}
$$

of simplicially stratified fibrations (defined as in 1.6.1) induces a map of $\Omega$ spectra $\mathbb{H}_{*}\left(X, \mathscr{S}_{*}(\mathrm{pt})\right) \rightarrow \mathbb{H}_{*}\left(\mathscr{B}(X), \mathscr{S}_{*}(\rho)\right)$, which is rationally injective on the homotopy group level, and then by applying 1.6. For details the reader is referred to Remark A.11 of the appendix.

1.6.3. The generalized Borel conjecture. This conjecture states that if $X$ is a closed compact aspherical manifold and $\tau$ is the total space of a bundle over $X$ whose fiber is a closed ball, then any homotopy equivalence $h:(M, \partial M) \rightarrow$ $(\tau, \partial \tau)$ from a compact manifold pair $(M, \partial M)$, with $h: \partial M \rightarrow \partial \tau$ already a homeomorphism, must be homotopic rel $\partial$ to a homeomorphism. (When $\tau=X$ we get Borel's original conjecture.) It is known that if $\operatorname{dim}(M) \geq 5$ then the generalized Borel conjecture is equivalent to the following two properties (cf. [36]): the usual assembly map $\mathbb{H}_{*}\left(X, \mathscr{L}_{*}^{h}(\mathrm{pt})\right) \rightarrow \mathscr{L}_{*}^{h}(X)$ is an equivalence of $\Omega$-spectra; $W h(\pi, X)=0$. Thus the generalized Borel conjecture (in dimensions $\geq 5$ ) would follow from 1.6 (cf. 1.6.1(a), (b)).

1.6.4. The role of the finite subgroups of $\pi_{1} X$. Several authors have recognized the role that the finite subgroups of $\pi_{1} X$ should play in any calculation of $\mathscr{S}_{*}(X)$. We shall review a few of these results now in terms of 
our own constructions and notation. Let $\mathscr{G}^{\prime}(X)$ denote the collection of all finite subgroups of $\pi_{1}(X)$. By using $\mathscr{G}^{\prime}(X)$ in place of $\mathscr{G}(X)$ in 1.5 we obtain the simplicially stratified fibration $\rho^{\prime}: \mathscr{E}^{\prime}(X) \rightarrow \mathscr{B}^{\prime}(X)$ and the map $f^{\prime}: \mathscr{E}^{\prime}(X) \rightarrow X$ in place of the maps $\rho: \mathscr{E}(X) \rightarrow \mathscr{B}(X)$ and $f: \mathscr{E}(X) \rightarrow X$. Let $A_{*}^{\prime}: \mathbb{H}_{*}\left(\mathscr{B}^{\prime}(X), \mathscr{S}_{*}\left(\rho^{\prime}\right)\right) \rightarrow \mathscr{S}_{*}\left(\mathscr{E}^{\prime}(X)\right)$ denote the assembly map for the simplicially stratified fibration $\rho^{\prime}: \mathscr{E}^{\prime}(X) \rightarrow \mathscr{B}^{\prime}(X)$. It is shown by Yamasaki $[57,58]$ that the composite map

$$
\text { (a) } \mathbb{H}_{*}\left(\mathscr{B}^{\prime}(X), \mathscr{S}_{*}\left(\rho^{\prime}\right)\right) \stackrel{\mathscr{P}_{*}\left(f^{\prime}\right) \circ A_{*}^{\prime}}{\longrightarrow} \mathscr{S}_{*}(X)
$$

is an equivalence when tensored with $\mathbf{Z}\left[\frac{1}{2}\right]$ for $\mathscr{S}_{*}()=\mathscr{L}_{*}^{-\infty}()$ in the special case when $\pi_{1} X$ is a virtually poly-Z group. It seems reasonable to conjecture that the map of (a) is always an equivalence when tensored with $\mathbf{Z}\left[\frac{1}{2}\right]$ for $\mathscr{S}_{*}($ ) equal to $\mathscr{L}_{*}^{-\infty}\left(\right.$ ) and is always a rational equivalence for $\mathscr{S}_{*}()=\mathscr{K}_{*}($ ) (in fact, a special case of this conjecture appears in [51]). This conjecture would certainly be a consequence of 1.6 (see Remark A.11 of the appendix for details). Hence Corollary 2.1.1 verifies it in certain cases where $\mathscr{S}_{*}()=\mathscr{K}_{*}()$.

The nontriviality of the nil-groups in algebraic $K$-theory and in surgery $L^{-\infty}$ theory prevents the map of (a) from being an equivalence in general (cf. [ 3 , 7]). However, a reasonable conjecture is that the map of (a) is always a split monomorphism of $\Omega$-spectra, for $\mathscr{S}_{*}$ equal to $\mathscr{P}_{*}, \mathscr{P}_{*}^{\text {diff }}, \mathscr{K}_{*}$, or $\mathscr{L}_{*}^{-\infty}$.

There is a conjecture due to Baum and Connes [3a, $3 \mathrm{~b}]$ which is quite similar to the conjectures we have just made. Paul Baum has informed the authors that the Baum-Connes conjecture (for discrete groups) is equivalent to requiring the map in (a) above to be an equivalence when $\mathscr{S}_{*}()$ is defined as follows. Set $\Gamma=\pi_{1} X$, let $C_{r}^{*} \Gamma$ denote the reduced $C^{*}$-algebra of $\Gamma$, and set $\mathscr{S}_{2 i+1}(X)=$ $G L\left(C_{r}^{*} \Gamma\right)$ and $\mathscr{S}_{2 i}(X)=\Omega \mathscr{S}_{2 i+1}(X)$.

1.6.5. Vanishing of the lower $K$-groups. We will show that if Conjecture 1.6 is true for the connected CW-complex $X$ and for $\mathscr{S}_{*}()=\mathscr{P}_{*}()$ then the following must hold. Set $\Gamma=\pi_{1} X$.

(a) $K_{i}(\mathbf{Z} \Gamma)=0$ for all integers $i \leq-2$.

(b) $K_{-1}(\mathbf{Z} \Gamma)$ is generated by the images of $K_{-1}(\mathbf{Z} F)$, where $F$ varies over all conjugacy classes of finite subgroups of $\Gamma$.

Toward verifying (a), (b) we first note that these properties hold when $\Gamma$ is replaced by any group in $\mathscr{G}(X)$ (cf. [26]). Next recall that $\pi_{i-2}\left(\mathscr{P}_{*}(X)\right)=$ $K_{i}(\mathbf{Z} \Gamma)$ for all integers $i \leq-1$ (cf. [1]). Finally recall that there is a spectral sequence, by 1.4 .2 , with $E_{i, j}^{2}=H_{i}\left(\mathscr{B}(X), \pi_{j}\left(\mathscr{P}_{*}(\rho)\right)\right)$ which converges to $H_{i+j}\left(\mathscr{B}(X), \mathscr{P}_{*}(\rho)\right)$, where $\pi_{j}\left(\mathscr{P}_{*}(\rho)\right)$ denotes the stratified system of groups $\left\{\pi_{j}\left(\mathscr{P}_{*}\left(\rho^{-1}(b)\right)\right): b \in \mathscr{B}(X)\right\}$ over $\mathscr{B}(X)$. Now using all these facts, together with 1.6 for $X$, we deduce that (a) and (b) hold for $\Gamma$.

We remark that (a) was proven for $\Gamma$ equal to any finite group by Carter [8] and was conjectured to hold for $\Gamma$ equal to any finitely presented group by W.-c. Hsiang [30]. As a corollary of Theorem 2.1 we get that (a) and (b) are both true for $\Gamma$ equal to any co-compact discrete subgroup of a virtually connected Lie group. 
1.6.6. The truth of 1.6 for any one of $\mathscr{P}_{*}(), \mathscr{P}_{*}^{\text {diff }}(), \mathscr{K}_{*}()$ implies it for the others? Recent work of Tom Goodwillie [28] implies that Conjecture 1.6 is true for the functor $\mathscr{P}_{*}()$ if and only if it is true for the functor $\mathscr{K}_{*}()$, on any aspherical CW-complex $X$ with $\pi_{1} X$ torsionfree. It seems likely that Goodwillie will be able to drop the hypotheses that $X$ be aspherical and that $\pi_{1} X$ be torsionfree.

An older result of Burghelea and Lashof [6] states that there is a fibration

$$
\mathscr{P}_{0}^{\text {diff }}() \rightarrow \mathscr{P}_{0}() \rightarrow \mathscr{P}^{S}(),
$$

where $\mathscr{P}^{S}()$ is a space-valued homology theory. It seems likely that their theory extends to $\Omega$-spectra to give a fibration

$$
\mathscr{P}_{*}^{\text {diff }}() \rightarrow \mathscr{P}_{*}() \rightarrow \mathscr{P}^{S}(),
$$

where $\mathscr{P}_{*}^{S}()$ is an $\Omega$-spectra-valued homology theory. Based on this extension, the authors envision the following proof that Conjecture 1.6 is true for $\mathscr{P}_{*}($ ) if and only if it is true for $\mathscr{P}_{*}^{\text {diff }}()$ : Conjecture 1.6 is true for $\mathscr{P}_{*}^{S}()$ since $\mathscr{P}_{*}{ }^{*}()$ is a homology theory; if any two functors in the fibration $\mathscr{P}_{*}^{\text {diff }}() \rightarrow \mathscr{P}_{*}() \rightarrow \mathscr{P}_{*}{ }^{*}()$ satisfy Conjecture 1.6, then so does the third functor.

1.6.7. Conjecture 1.6 is true for $\mathscr{P}^{\mathrm{diff}}() \otimes \mathbf{Q}$ if and only if it is true for $\mathscr{K}_{*}() \otimes \mathbf{Q}$, for $X$ equal to any aspherical $\mathbf{C W}$-complex. This result would follow from the generalization of Goodwillie's result envisioned in 1.6.6. Here we give a proof based on well-established results of Anderson and Hsiang [1] and Waldhausen [54]. Let $\mathscr{C}$ denote the category of all spaces homotopy equivalent to aspherical $\mathrm{CW}$-complexes, with all continuous maps between such spaces. The following claim can be deduced from the work of Anderson and Hsiang [1, Theorem 3] in dimensions $i \leq-1$ and from the work of Waldhausen [54, 2.8, 2.9, pp. 48, 51] in dimensions $i \geq 0$.

Claim. (a) For any space $A \in \mathscr{C}$ there is an isomorphism

$$
I_{A}:\left(\pi_{i} \mathscr{P}_{*}^{\text {diff }}(A) \otimes \mathbf{Q}\right) \oplus H_{i+2}(A, \mathbf{Q}) \rightarrow \pi_{i+2} \mathscr{K}_{*}(A) \otimes \mathbf{Q},
$$

where $H_{*}(, \mathbf{Q})$ denotes singular homology theory with rational coefficients.

(b) For any map $g: A \rightarrow B$ (in $\mathscr{C}$ ) the following diagram is commutative

$$
\begin{array}{cc}
\left(\pi_{i} \mathscr{P}_{*}^{\text {diff }}(A) \otimes \mathbf{Q}\right) \oplus H_{i+2}(A, \mathbf{Q}) \stackrel{I_{A}}{\longrightarrow} \pi_{i+2} \mathscr{K}_{*}(A) \otimes \mathbf{Q} \\
\alpha \downarrow & \downarrow^{\alpha} \\
\left(\pi_{i} \mathscr{P}_{*}^{\text {diff }}(B) \otimes \mathbf{Q}\right) \oplus H_{i+2}(B, \mathbf{Q}) \stackrel{I_{B}}{\longrightarrow} \pi_{i+2} \mathscr{K}_{*}(B) \otimes \mathbf{Q}
\end{array}
$$

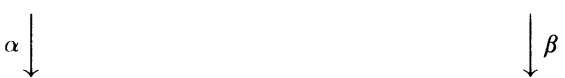

where $\alpha=\left(\pi_{i} \mathscr{P}_{*}^{\text {diff }}(g) \otimes \mathbf{Q}\right) \oplus H_{i+2}(g, Q)$ and $\beta=\pi_{i+2} \mathscr{K}_{*}(g) \otimes \mathbf{Q}$.

Let $\mathbb{H}_{*}(A, \mathbf{Q})$ denote the homology spectrum for the space $A$ associated to singular homology theory with rational coefficients. Since both $\left(\mathscr{P}_{*}^{\text {diff }}(A) \otimes \mathbf{Q}\right) \oplus$ $\mathbb{H}_{*-2}(A, \mathbf{Q})$ and $\mathscr{K}_{*-2}(A) \otimes \mathbf{Q}$ are products of Eilenberg-MacLane $\Omega$-spectra (for all spaces $A \in \mathscr{C}$ ), it follows that the isomorphism of Claim(a) is induced by a map $J_{A}:\left(\mathscr{P}_{*}^{\mathrm{diff}}(A) \otimes \mathbf{Q}\right) \oplus \mathbb{H}_{*-2}(A, \mathbf{Q}) \rightarrow \mathscr{K}_{*-2}(A) \otimes \mathbf{Q}$ between $\Omega$-spectra, 
which is unique up to homotopy. It follows from Claim(b) that for any map $g: A \rightarrow B$ in $\mathscr{C}$ the diagram

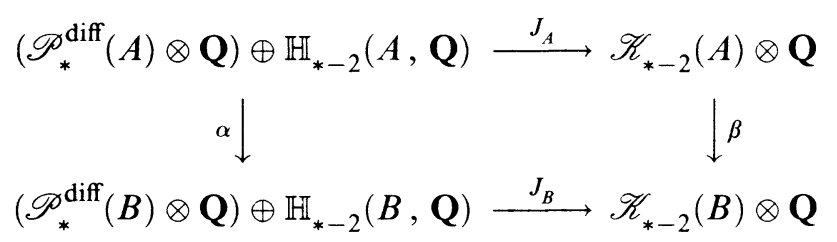

is homotopy commutative, where $\alpha=\left(\mathscr{P}_{*}^{\text {diff }}(g) \otimes \mathbf{Q}\right) \oplus \mathbb{H}_{*-2}(g, \mathbf{Q})$ and $\beta=$ $\mathscr{K}_{*-2}(g) \otimes \mathbf{Q}$. Let $K$ denote the canonical triangulation of $\mathscr{B}(X)$, and let $K^{(1)}$ denote its first barycentric subdivision. It follows that the maps $\left\{J_{A}: A=\right.$ $\left.\rho^{-1}(\Delta), \Delta \in K^{(1)}\right\}$ induce an equivalence of $\Omega$-spectra

$$
J: \mathbb{H}_{*}\left(\mathscr{B}(X),\left(\mathscr{P}_{*}^{\text {diff }}(\rho) \otimes \mathbf{Q}\right) \oplus \mathbb{H}_{*-2}(\rho, \mathbf{Q})\right) \rightarrow \mathbb{H}_{*}\left(\mathscr{B}(X), \mathscr{K}_{*-2}(\rho) \otimes \mathbf{Q}\right)
$$

such that the diagram

$$
\begin{gathered}
\mathbb{H}_{*}\left(\mathscr{B}(X),\left(\mathscr{P}_{*}^{\text {diff }}(\rho) \otimes \mathbf{Q}\right) \oplus \mathbb{H}_{*-2}(\rho, \mathbf{Q})\right) \stackrel{J}{\longrightarrow} \mathbb{H}_{*}\left(\mathscr{B}(X), \mathscr{K}_{*-2}(\rho) \otimes \mathbf{Q}\right) \\
\left(\mathscr{P}_{*}^{\text {diff }}(X) \otimes \mathbf{Q}\right) \oplus \mathbb{H}_{*-2}(X, \mathbf{Q}) \stackrel{J_{X}}{\longrightarrow} \quad \mathscr{K}_{*-2}(X) \otimes \mathbf{Q}
\end{gathered}
$$

is commutative, where $\alpha$ denotes the composite of the assembly map (for the simplicially stratified fibration $\rho$ and the functor $\left.\left(\mathscr{P}_{*}^{\text {diff }}() \otimes \mathbf{Q}\right) \oplus \mathbb{H}_{*-2}(, \mathbf{Q})\right)$ with the map $\left(\mathscr{P}_{*}^{\text {diff }}(f) \otimes \mathbf{Q}\right) \oplus \mathbb{H}_{*-2}(f, \mathbf{Q})$ (where $f: \mathscr{E}(X) \rightarrow X$ ) and $\beta$ denotes the composite of the assembly map (for the simplicially stratified fibration $\rho$ and the functor $\left.\mathscr{K}_{*-2}() \otimes \mathbf{Q}\right)$ with the map $\mathscr{K}_{*-2}(f) \otimes \mathbf{Q}$. Since both $J$ and $J_{X}$ are equivalences, the proof of 1.6.7 is finished.

1.7. The fibered isomorphism conjectures. When trying to verify special cases of 1.6 in the following sections it will be useful to actually verify a more general fibered version of 1.6. This will allow us to use fibration induction arguments (cf. $\S \S 2$ and 4), a useful tool for verifying special cases of 1.6. We will now formulate the fibered version of 1.6.

Let $X$ be a connected $C W$-complex, and let $\xi=Y \rightarrow X$ denote a Serre fibration over $X$. Let $\mathscr{E}(\xi)$ denote the total space of the pullback of $\xi$ along the map $f: \mathscr{E}(X) \rightarrow X$, let $\rho(\xi): \mathscr{E}(\xi) \rightarrow \mathscr{B}(\xi)$ denote the composite map $\mathscr{E}(\xi) \stackrel{\text { proj }}{\rightarrow} \mathscr{E}(X) \stackrel{\rho}{\rightarrow} \mathscr{B}(X)$, and let $f(\xi): \mathscr{E}(\xi) \rightarrow Y$ denote the map which covers the map $f: \mathscr{E}(X) \rightarrow X$. We conjecture that the composite map

$$
\mathbb{H}_{*}\left(\mathscr{B}(\xi), \mathscr{S}_{*}(\rho(\xi))\right) \stackrel{\mathscr{S}_{*}(f(\xi)) \circ A_{*}}{\longrightarrow} \mathscr{S}_{*}(Y)
$$

is an equivalence of $\Omega$-spectra, where $\mathscr{S}_{*}()$ denotes any one of the functors $\mathscr{P}_{*}(), \mathscr{P}_{*}^{\text {diff }}(), \mathscr{K}_{*}()$, or $\mathscr{L}_{*}^{-\infty}()$, where $A_{*}$ is the assembly map for the simplicially stratified fibration ${ }^{*} \rho(\xi): \mathscr{E}(\xi) \rightarrow \mathscr{B}^{*}(\xi)$ and $\mathscr{S}_{*}(f(\xi))$ is the image of the map $f(\xi): \mathscr{E}(\xi) \rightarrow Y$ under the functor $\mathscr{S}_{*}()$. 
Remark 1.7.1. For the reasons stated in 1.6.6 the authors believe that if Conjecture 1.7 can be verified for any one of the functors $\mathscr{P}_{*}^{\text {diff }}(), \mathscr{P}_{*}(), \mathscr{K}_{*}()$ then it should follow for the other two functors. A modified version of the argument used in 1.6.7 shows that Conjecture 1.7 is true for the functor $\mathscr{P}_{*}^{\text {diff }}() \otimes Q$ if and only if it is true for the functor $\mathscr{K}_{*}() \otimes Q$ for all fibrations $\xi$ whose fiber, base, and total space are all aspherical.

\section{The MAIN THEOREM AND AN OUTLINE OF PROOF}

In this section we state the main result of the paper (cf. 2.1). We also formulate three propositions (cf. 2.2, 2.3, 2.4) and then deduce the main result from these propositions. These propositions are proven in $\S \S 3,4$ and the appendix.

2.1. Theorem. Let $X$ be a connected $C W$-complex such that $\pi_{1} X$ is a cocompact discrete subgroup of a virtually connected Lie group. Then the Fibered Conjecture 1.7 is true for the functors $\mathscr{P}_{*}()$ and $\mathscr{P}_{*}^{\text {diff }}()$ on the space $X$.

The following corollary is deduced from Theorem 2.1 (together with the part of Remark A.11 of the Appendix pertaining to 1.6.4) and from 1.6.7, 1.7.1.

2.1.1. Corollary. The map $\mathscr{K}_{*}(f(\xi)) \circ A_{*}: \mathbb{H}_{*}\left(\mathscr{B}(\xi), \mathscr{K}_{*}(\rho(\xi))\right) \rightarrow \mathscr{K}_{*}(Y)$ of 1.7 is a rational equivalence of $\Omega$-spectra for any Serre fibration $\xi=Y \rightarrow X$ over a space $X$ as in 2.1, provided $X, Y$ and the homotopy fiber of $\xi$ are all aspherical. Consequently the map of $1.6 .4(\mathrm{a})$ is a rational equivalence (as was conjectured in 1.6.4) for $\mathscr{S}_{*}()=\mathscr{K}_{*}()$ and $X$ is as in 2.1.

2.1.2. Remark. Note that if Goodwillie's result can be generalized as suggested in 1.7.1, then Theorem 2.1 would imply that the map of 2.1 .1 is an equivalence. It seems likely that this could also be proven by the arguments of $\S \S 3,4$, suitably adapted to algebraic $K$-theory (instead of pseudoisotopy theory).

2.1.3. Remark. We can also prove a version of Theorem 2.1 for the functor $\mathscr{S}_{*}()=\mathscr{L}_{*}^{-\infty}()$. The proof is just a fibered equivariant version of the arguments given in [25]. The proof of Theorem 2.1 given below (which is just a fibered equivariant version of the arguments given in [24]) should serve as a model for how to carry out the fibered equivariant version of the arguments in [25].

We will need the following notation in the next proposition. Let $p: E \rightarrow X$ denote a Serre fibration such that both $X$ and $E$ are connected $C W$-complexes. Also assume that $P_{\#}: \pi_{1} E \rightarrow \pi_{1} X$ is an epimorphism. Let $\mathscr{G}(X)$ denote the class of subgroups of $\pi_{1}(X)$ given in 1.5. This next proposition is proven in Remark A.12 of the appendix.

2.2. Proposition. The Fibered Conjecture 1.7 is true for any of the functors $\mathscr{S}_{*}\left(\right.$ ) of 1.1-1.3 on the total space of the fibration $p: E \rightarrow X$ if it is true for $\mathscr{F}_{*}($ ) on each of the following spaces: the space $X$; and any connected $C W$-complex $Z$ such that $\pi_{1} Z$ is isomorphic to $p_{\#}^{-1}(H)$ for some group $H \in \mathscr{G}(X)$.

2.2.1. Corollary. Let $X$ be a connected $C W$-complex, and let $C$ be any connected aspherical $C W$-complex with $\pi_{1} C=\pi_{1} X$. Then the Fibered Conjecture 
1.7 is true for the functor $\mathscr{S}_{*}()$ on the space $X$ if it is true for the functor $\mathscr{P}_{*}()$ on the space $C$.

Proof of Corollary 2.2.1. Let $\widehat{C}, \widehat{X}$ denote the universal covering spaces for $C, X$. Since $\pi_{1} C=\pi_{1} X$, the group $\pi_{1} C$ acts by deck transformations on both $\widehat{C}, \widehat{X}$. Let $\pi_{1} C \times(\widehat{C} \times \widehat{X}) \rightarrow \widehat{C} \times \widehat{X}$ denote the diagonal action, and let $p: E \rightarrow C$ denote the quotient of the standard projection $\widehat{C} \times \widehat{X} \rightarrow \widehat{C}$ under the $\pi_{1} C$-actions. Note that $p: E \rightarrow C$ is a fiber bundle with simply connected fiber. The hypothesis of 2.2 is satisfied for any fiber bundle with simply connected fiber. So, if we assume that the Fibered Conjecture 1.7 holds for $\mathscr{S}_{*}()$ on $C$, then we conclude from 2.2 that it also holds true for $\mathscr{S}_{*}()$ on $E$. Finally we note that $E$ and $X$ are homotopy equivalent. So the truth of the Fibered Conjecture 1.7 for $\mathscr{S}_{*}(\mathrm{)}$ on one of the spaces $X, E$ implies the truth for the other space.

This completes the proof of Corollary 2.2.1.

The following two propositions are proven in $\S \S 3,4$ respectively.

2.3. Proposition. Let $X$ be a connected $C W$-complex, and let $M$ denote a simply connected symmetric Riemannian manifold with sectional curvature $K \leq$ 0 everywhere. Suppose that there is a properly discontinuous co-compact group action $\pi_{1} X \times M \rightarrow M$ by isometries of $M$. Then the Fibered Conjecture 1.7 is true for the functors $\mathscr{P}_{*}()$ and $\mathscr{P}_{*}^{\text {diff }}($ ) on the space $X$.

2.4. Proposition. Let $X$ be a connected $C W$-complex. Suppose that $\pi_{1} X$ is a virtually poly-Z group. Then the Fibered Conjecture 1.7 is true for the functors $\mathscr{P}_{*}\left(\right.$ ) and $\mathscr{P}_{*}^{\text {diff }}()$ on the space $X$.

Proof of Theorem 2.1. We break the proof into the following three steps. (Compare [13] and [14] for related reductions.) Let $G$ denote the virtually connected Lie group which contains a co-compact discrete subgroup $\Gamma$ isomorphic to $\pi_{1} X$. For any Lie group $L$ we denote by $L_{e}$ the identity component of $L$.

Step 1. In this step we show that we may, without loss of generality, assume that $G$ has no nontrivial compact connected normal subgroups. Let $C$ denote the maximal connected compact normal subgroup of $G$. Let $q: G \rightarrow G^{\prime}$ denote the quotient map onto $G^{\prime}=G / C$, and set $\Gamma^{\prime}=q(\Gamma), \Gamma_{C}=\Gamma \cap C$. Note that $\Gamma^{\prime}$ is a co-compact discrete subgroup of $G^{\prime}$ and that $G^{\prime}$ is a virtually connected Lie group having no nontrivial compact connected normal subgroups. Thus, to complete Step 1 it will suffice to show that if the Fibered Conjecture 1.7 is true for the aspherical space $K\left(\Gamma^{\prime}, 1\right)$ then it is also true for $X$. To see this we choose a fiber bundle sequence $K\left(\Gamma_{C}, 1\right) \rightarrow K(\Gamma, 1) \rightarrow K\left(\Gamma^{\prime}, 1\right)$ of aspherical cell complexes. (This is possible since there is the short exact sequence of groups $1 \rightarrow \Gamma_{C} \rightarrow \Gamma \rightarrow \Gamma^{\prime} \rightarrow 1$; cf., Remark A5.1 in the appendix.) If the Fibered Conjecture were true for $K\left(\Gamma^{\prime}, 1\right)$, we could apply Proposition 2.2 (note that the hypotheses of 2.2 are satisfied because $\Gamma_{C}$ is finite) and Corollary 2.2.1 to this sequence to conclude that the Fibered Conjecture 1.7 is true for the space $X$.

Step 2. In this step we verify Theorem 2.1 for the special case that $G_{e}$ is a semisimple Lie group. By Step 1 we may assume that $G$ contains no nontrivial compact connected normal subgroups. Let $Z$ denote the subgroup 
of all elements of $G$ which commute with every element in $G_{e}$. Let $q: G \rightarrow$ $G / Z=G^{\prime}$ denote the quotient map, and set $\Gamma_{\mathrm{Z}}=\Gamma \cap Z, \Gamma^{\prime}=q(\Gamma)$. Since $G$ contains no nontrivial compact connected normal subgroups, we may apply [42, Corollary 5.17] in verifying that the following properties are true.

2.5. (a) There is a short exact sequence of groups $1 \rightarrow \Gamma_{Z} \rightarrow \Gamma \rightarrow \Gamma^{\prime} \rightarrow 1$.

(b) $\Gamma_{Z}$ is a finite extension of a finitely generated abelian group.

(c) $\Gamma^{\prime}$ is a co-compact discrete subgroup of $G^{\prime}$.

(d) $G^{\prime}$ is a virtually connected linear Lie group such that $G_{e}^{\prime}$ is semisimple.

Consider the fiber bundle sequence $K\left(\Gamma_{\mathrm{Z}}, 1\right) \rightarrow K(\Gamma, 1) \rightarrow K\left(\Gamma^{\prime}, 1\right)$ of aspherical cell complexes. By applying Propositions 2.2 and 2.4 to this sequence, in conjunction with $2.5(\mathrm{a})$, (b), we conclude that the Fibered Conjecture 1.7 is true for $K(\Gamma, 1)$ provided it is true for $K\left(\Gamma^{\prime}, 1\right)$. Thus, by Corollary 2.2.1, the Fibered Conjecture would also be true for $X$ if it were true for $K\left(\Gamma^{\prime}, 1\right)$. On the other hand, it follows from $2.5(\mathrm{c})$, (d) that $\Gamma^{\prime}$ acts properly discontinuously and co-compactly by isometries on a simply connected Riemannian symmetric space having nonpositive sectional curvature (i.e., on $G^{\prime} / C$, where $C$ is a maximal compact subgroup of $G^{\prime}$ ). So by Proposition 2.3 the Fibered Conjecture is true for $K\left(\Gamma^{\prime}, 1\right)$.

Step 3. In this step we complete the proof of Theorem 2.1. By Step 1 we may assume that $G$ contains no nontrivial compact connected normal subgroups. Let the short exact sequence of Lie groups $1 \rightarrow R \rightarrow G \stackrel{L}{\rightarrow} S \rightarrow 1$ denote the Levi decomposition of $G$; that is, $R$ is the maximal (closed) connected normal solvable subgroup of $G$ and $S=G / R$ is a virtually connected Lie group with $S_{e}$ semisimple (cf. [42]). Set $\Gamma_{R}=\Gamma \cap R$ and $\Gamma_{S}=L(\Gamma)$. Since $G$ contains no connected compact normal subgroups, it follows that we may apply $[42,8.28]$ to conclude that $\Gamma_{R}, \Gamma_{S}$ are co-compact discrete subgroups of $R, S$. We sum up these properties as follows.

2.6. (a) $\Gamma_{R}$ is a virtually poly- $Z$ group. (Here we use Mostow's result that any lattice in a connected solvable Lie group must be a virtually poly- $Z$ group (cf. [42, Chapter 3]).)

(b) $\Gamma_{S}$ is a co-compact discrete subgroup of a virtually connected Lie group $S$ with $S_{e}$ semisimple.

(c) There is a short exact sequence of groups $1 \rightarrow \Gamma_{R} \rightarrow \Gamma \rightarrow \Gamma_{S} \rightarrow 1$.

Now, by applying Step 2, in conjunction with $2.6(\mathrm{~b})$, it follows that the Fibered Conjecture 1.7 is true for the aspherical space $K\left(\Gamma_{S}, 1\right)$. Next consider the fiber bundle sequence $K\left(\Gamma_{R}, 1\right) \rightarrow K(\Gamma, 1) \rightarrow K\left(\Gamma_{S}, 1\right)$ of aspherical cell complexes. Since the Fibered Conjecture is true for $K\left(\Gamma_{S}, 1\right)$, we may apply Propositions 2.2 and 2.4 and Corollary 2.2.1 to this sequence, in conjunction with 2.6(a), (c), to conclude that the Fibered Conjecture is true for the space $X$.

\section{Proof of Proposition 2.3}

Our hypothesis in this section is that of Proposition 2.3, that there exists a properly discontinuous and co-compact group action $\pi_{1} X \times M \rightarrow M$ by 
isometries of $M$, where $M$ is a simply connected Riemannian symmetric space having all sectional curvatures $K \leq 0$. A straightforward application of Proposition 2.2 allows us to make the additional assumption that this action is faithful. In this section we shall use the shorter notation $\rho: \mathscr{E}(\xi) \rightarrow \mathscr{B}(\xi)$ for the map $\rho(\xi): \mathscr{E}(\xi) \rightarrow \mathscr{B}(\xi)$. We begin by giving an explicit geometric construction for $\rho: \mathscr{E}(\xi) \rightarrow \mathscr{B}(\xi)$. We use the special notation $\bar{\rho}: \overline{\mathscr{E}}(\xi) \rightarrow \overline{\mathscr{B}}(\xi)$ for this explicit representative of the fibered homotopy type $\rho: \mathscr{E}(\xi) \rightarrow \mathscr{B}(\xi)$.

3.1. The simplicially stratified fibration $\bar{\rho}: \overline{\mathscr{E}}(\xi) \rightarrow \overline{\mathscr{B}}(\xi)$. Let $S M$ and $R P M$ denote the unit sphere bundle and the real projective bundle for $M$, and let $\mathscr{F}$ denote the one-dimensional smooth foliation of $R P M$ whose leaves are covered by the orbits of the geodesic flow $g^{t}: S M \rightarrow S M, t \in R$, under the twofold covering projection $S M \rightarrow R P M$. Let $p: R P M \rightarrow M$ denote the standard projection, and let $q: M \rightarrow M / \pi_{1} X$ denote the quotient map. We denote by $E \subset R P M$ the union of all the leaves $L \in \mathscr{F}$ such that $q \circ p(L)$ is a compact subset of $M / \pi_{1} X$. We have proven in $[24,2.4,2.15,2.16,4.0]$ that $E$ has a countable number of path components $E_{1}, E_{2}, E_{3}, \ldots$, each of which is a smooth submanifold of $R P M$ having no manifold boundary, and each of which is a closed subset of $R P M$. (Actually this is proven in [24] under the hypothesis that $\pi_{1} X$ is a torsionfree group. If $\pi_{1} X$ is not torsionfree then there is, by a well-known result due to Selberg, a torsionfree normal subgroup $\Gamma \subset \pi_{1} X$ of finite index, and $M / \Gamma$ is compact since $M / \pi_{1} X$ is compact. In this way the statement about $E$ follows from [24].) For future reference we let $F, F_{i}$ denote the restriction of $\mathscr{F}$ to $E, E_{i}$. Choose an integer $a>\operatorname{dim} M$, and let $\pi: M \times \mathbf{R}^{a} \rightarrow M$ denote the standard projection. Choose smooth embeddings $g_{i}: E_{i} \rightarrow M \times \mathbf{R}^{a}, i=1,2,3, \ldots$, with closed images, which satisfy the following properties.

3.1.1. (a) The union $\bigcup_{i} g_{i}: E \rightarrow M \times \mathbf{R}^{a}$ is a one-to-one function.

(b) $\pi \circ g_{i}=p \mid E_{i}$.

(c) Note that $\pi_{1} X: M \rightarrow M$ induces an action $\pi_{1} X \times R P M \rightarrow R P M$ which permutes the leaves of $F$ and thus restricts to an action $\pi_{1} X \times E \rightarrow E$. We require that $\pi_{1} X$ acts through a finite quotient group $G$ by linear isometries on $\mathbf{R}^{a}$; let $\pi_{1} X \times\left(M \times \mathbf{R}^{a}\right) \rightarrow M \times \mathbf{R}^{a}$ denote the diagonal action. We also require that the union $\bigcup_{i} g_{i}: E \rightarrow M \times \mathbf{R}^{a}$ be a $\pi_{1} X$-equivariant map.

(d) Let $S_{1}, S_{2}, S_{3}, \ldots$ denote the equivalence classes of the path components of $E$, where $E_{i}$ is equivalent to $E_{j}$ if and only if there is $\alpha \in \pi_{1} X$ such that $E_{i}=\alpha E_{j}$. Then for each $i=1,2,3, \ldots$ and for each $E_{j} \in S_{i}$ we must have that Image $\left(g_{j}\right) \subset M \times\left(B_{2 i}^{a}-B_{2 l-1}^{a}\right)$, where $B_{r}^{a}$ denotes the ball of radius $r$ centered at the origin in $\mathbf{R}^{a}$.

3.1.1.1. Remark. To get the embeddings $g_{i}: E_{i} \rightarrow M \times \mathbf{R}^{a}$ of 3.1 .1 and the action $G \times \mathbf{R}^{a} \rightarrow \mathbf{R}^{a}$ of 3.1.1(c) we proceed as follows. By Selberg [45] we may choose a torsionfree normal subgroup $\Gamma \subset \pi_{1} X$ of finite index such that the restricted action $\Gamma \times M \rightarrow M$ is by the deck transformations of a covering space $M \rightarrow M / \Gamma$. Let $N$ denote the compact locally symmetric space $M / \Gamma$ and $G=\pi_{1} X / \Gamma$. Note that there is an action $G \times N \rightarrow N$ by isometries of $N$. 
which induces an action $G \times R P N \rightarrow R P N$. The Mostow-Palais embedding theorem [37, 41] states that for a sufficiently large integer $a$ there is an action $G \times \mathbf{R}^{a} \rightarrow \mathbf{R}^{a}$ by linear isometries and a smooth $G$-equivariant embedding $h: R P N \rightarrow B_{1}^{a} \subset \mathbf{R}^{a}$. Now the embeddings $g_{i}: E_{i} \rightarrow M \times \mathbf{R}^{a}$ may be defined to be the Cartesian product of the composite maps $E_{i} \subset R P M \stackrel{\text { proj }}{\longrightarrow} M$ with the composite maps $E_{i} \subset R P M \stackrel{\text { proj }}{\longrightarrow} R P N \stackrel{h}{\rightarrow} \mathbf{R}^{a}$. The embeddings thus constructed satisfy $3.1 .1(\mathrm{a})-(\mathrm{c})$ but not $3.1 .1(\mathrm{~d})$. In order to satisfy $3.1 .1(\mathrm{~d})$ we replace the action $G \times \mathbf{R}^{a} \rightarrow \mathbf{R}^{a}$ by the diagonal action $G \times \mathbf{R}^{a} \times \mathbf{R} \rightarrow \mathbf{R}^{a} \times \mathbf{R}$ (where $G$ acts trivially on the factor $\mathbf{R})$ and replace the embeddings $g_{i}: E_{i} \rightarrow M \times \mathbf{R}^{a}$ by the composite maps $E_{i} \stackrel{g_{i}}{\longrightarrow} M \times \mathbf{R}^{a}=M \times \mathbf{R}^{a} \times 0 \subset M \times \mathbf{R}^{a} \times \mathbf{R} \stackrel{T_{i}}{\rightarrow} M \times \mathbf{R}^{a} \times \mathbf{R}$, where the $T_{i}$ denote suitable translations in the $\mathbf{R}$-factor.

Since we will use the embeddings $g_{i}: E_{i} \rightarrow M \times \mathbf{R}^{a}$ a lot, we will often denote them by $E_{i} \subset M \times \mathbf{R}^{a}$. Let $u: M \times \mathbf{R}^{a} \rightarrow M^{\prime}$ denote the quotient map obtained by collapsing to a point each leaf of $F$. Note that $\pi_{1} X \times\left(M \times \mathbf{R}^{a}\right) \rightarrow M \times \mathbf{R}^{a}$ induces an action $\pi_{1} X \times M^{\prime} \rightarrow M^{\prime}$.

Let $\xi$ denote a fibration $Y \rightarrow X$ over $X$, and let $\hat{\xi}=\hat{Y} \rightarrow \widehat{X}$ denote the pullback of $\xi$ along the universal covering projection $\hat{X} \rightarrow X$. Note that there is a free group action $\pi_{1} X \times \widehat{Y} \rightarrow \widehat{Y}$, and let $\pi_{1} X \times\left(M^{\prime} \times \widehat{Y}\right) \rightarrow M^{\prime} \times \widehat{Y}$ denote the diagonal action. We define the map $\bar{\rho}: \overline{\mathscr{E}}(\xi) \rightarrow \overline{\mathscr{B}}(\xi)$ to be the quotient of the composite map $\left(M \times \mathbf{R}^{a}\right) \times \widehat{Y} \stackrel{u \times \text { id }}{\longrightarrow} M^{\prime} \times \widehat{Y} \stackrel{\text { proj }}{\longrightarrow} M^{\prime}$ under the $\pi_{1} X$-actions. We define a map $\bar{f}: \overline{\mathscr{E}}(\xi) \rightarrow Y$ to be the quotient of the standard projection map $\left(M \times \mathbf{R}^{a}\right) \times \widehat{Y} \rightarrow \widehat{Y}$ under the relevant $\pi_{1} X$-actions.

3.1.2. Lemma. $M^{\prime}$ equipped with the above action $\pi_{1} X \times M^{\prime} \rightarrow M^{\prime}$ is a universal $\left(\pi_{1} X, \mathscr{G}(X)\right)$-space. Thus $\bar{\rho}: \overline{\mathscr{E}}(\xi) \rightarrow \overline{\mathscr{B}}(\xi)$ is a simplicially stratified fibration which represents the fibered homotopy type of $\rho: \mathscr{E}(\xi) \rightarrow \mathscr{B}(\xi)$. Moreover, $\bar{f}: \overline{\mathscr{E}}(\xi) \rightarrow Y$ is a homotopy equivalence.

Proof of Lemma 3.1.2. For each $E_{i}$ let $\Gamma_{i} \subset \pi_{1} X$ denote the subgroup which leaves $E_{i} \subset R P M$ invariant under the action $\pi_{1} X \times R P M \rightarrow R P M$. We have proven the following properties in $[24,2.7 .1,2.7 .2,2.12,2.13]$ under the assumption that $\pi_{1} X$ is torsionfree.

3.1.3. (a) There is a convex totally geodesic submanifold $D_{i} \subset M$ and an isometry $D_{i} \times \mathbf{R}=E_{i}$, where $D_{i} \times \mathbf{R}$ is equipped with the product metric.

(b) The foliation of $E_{i}$ by $F_{i}$ is the same under this isometry as the foliation of $E_{i}=D_{i} \times \mathbf{R}$ by the lines $\left\{d \times \mathbf{R}: d \in D_{i}\right\}$.

(c) The action $\Gamma_{i}: E_{i} \rightarrow E_{i}$ preserves the product structure $E_{i}=D_{i} \times \mathbf{R}$. For each $\alpha \in \Gamma_{i}$ let $\alpha_{1}: D_{i} \rightarrow D_{i}$ and $\alpha_{2}: \mathbf{R} \rightarrow \mathbf{R}$ be the isometries such that $\alpha=\alpha_{1} \times \alpha_{2}$, and set $\Gamma_{i, 1}=\left\{\alpha_{1}: \alpha \in \Gamma_{i}\right\}$. Then the action $\Gamma_{i, 1} \times D_{i} \rightarrow D_{i}$ is properly discontinuous.

(d) Each quotient $E_{i} / \Gamma_{i}$ is a compact manifold without boundary.

(e) For each $i$ there is an element $g_{i} \in \Gamma_{i}$ of infinite order and a positive integer $n_{i}$ such that $\left(g_{i}^{n_{i}}\right)_{1}=\mathrm{id} \mid D_{1}$ and $N\left(\left\langle g_{i}^{n_{i}}\right\rangle, \pi_{1} X\right)=\Gamma_{i}$, where $\langle g\rangle$ is the cyclic subgroup of a group $G$ generated by any group element $g \in G$ and 
$N\left(G_{1}, G_{2}\right)$ denotes the normalizer in the group $G_{2}$ for the subgroup $G_{1} \subset G_{2}$. Conversely, for each $g \in \pi_{1} X$ of infinite order there is a unique integer $i$ such that for some $p \in D_{i}$ we have that $g\left(p \times D_{i}\right)=p \times D_{i}$; for such an integer $i$ we also have that $g\left(E_{i}\right)=E_{i},\left(g^{n}\right)_{1}=\mathrm{id} \mid D_{i}$ for some positive integer $n$, and $N\left(\left\langle g^{n}\right\rangle, \pi_{1} X\right)=\Gamma_{i}$.

If $\pi_{1} X$ is not torsionfree, Selberg [45] provides us with a normal subgroup $\Gamma \subset \pi_{1} X$ which is torsionfree and has finite index in $\pi_{1} X$. Note that $\Gamma \times M \rightarrow$ $M$ is a properly discontinuous co-compact action by isometries of the simply connected symmetric space $M$. So by [24] we have that $3.1 .3(\mathrm{a})-(\mathrm{e})$ are true when $\pi_{1} X$ is replaced by $\Gamma$. Now, since $\pi_{1} X$ is a finite extension of $\Gamma$ and the action $\pi_{1} X \times M \rightarrow M$ is also by isometries of the simply connected symmetric space $M$, it can easily be argued that 3.1 .3 is also satisfied by the larger group $\pi_{1} X$, provided $3.1 .3(\mathrm{~d})$ is replaced by the following slightly weaker condition.

3.1.3. ( $\left.\mathrm{d}^{\prime}\right)$ Each $\Gamma_{i}$ contains a torsionfree normal subgroup $\pi_{i}$ with finite index. Furthermore, each quotient $E_{i} / \pi_{i}$ is a compact manifold without boundary.

Denoting $\pi_{1} X$ by $\Gamma$ and $\mathscr{G}(X)$ by $\mathscr{C}$, we now complete the proof of the first part of Lemma 3.1.2 (which claims that $\Gamma \times M^{\prime} \rightarrow M^{\prime}$ is a universal $(\Gamma, \mathscr{C})$-space) by verifying the following properties.

3.1.4. (a) $M^{\prime}$ can be equipped with a triangulation $K$ such that $\Gamma \times M^{\prime} \rightarrow M^{\prime}$ is a simplicial action. Moreover, if for $g \in \Gamma$ and $\Delta \in K$ we have that $g(\Delta)=\Delta$, then $g \mid \Delta=$ inclusion.

(b) For any $p \in M^{\prime}$ we have that $\Gamma^{p} \in \mathscr{C}$, where $\Gamma^{p}$ denotes the isotropy subgroup for the action $\Gamma \times M^{\prime} \rightarrow M^{\prime}$ at $p$.

(c) For each subgroup $H \in \mathscr{C}$ we have that $M^{\prime H}$ is a nonempty contractible subcomplex of $K$, where $M^{\prime H}$ denotes the fixed point set for the action $H \times$ $M^{\prime} \rightarrow M^{\prime}$.

To verify $3.1 .4(\mathrm{a})$ note that it follows from 3.1 .1 and 3.1 .3 that $M^{\prime} / \Gamma$ is a smoothly stratified space and can thus be triangulated. Now define $K$ to be a lifting to $M^{\prime}$ of a triangulation for $M^{\prime} / \Gamma$.

To verify $3.1 .4(\mathrm{~b})$ we first note that if $p \in M^{\prime}-u(E)$ then $\Gamma^{p}$ is a finite group, where $u: M \times \mathbf{R}^{a} \rightarrow M^{\prime}$ is the quotient map. On the other hand, if $p \in u\left(E_{i}\right)$, then $\Gamma^{p}=\{g \in \Gamma: g(q \times \mathbf{R})=q \times \mathbf{R}\}$, where $u^{-1}(p)=q \times \mathbf{R}$ under the isometry $E_{i}=D_{i} \times \mathbf{R}$ of $3.1 .3(\mathrm{a})$. Thus $\Gamma^{b}$ acts properly discontinuously and co-compactly on $q \times \mathbf{R}$ by isometries; consequently, $\Gamma^{p} \in \mathscr{C}$.

Finally we verify $3.1 .4(\mathrm{c})$. First consider the case where $H \in \mathscr{C}$ contains an element $g \in H$ of infinite order. Let $E_{i}$ be the path component of $E$ associated to $g$ by the second part of $3.1 .3(\mathrm{e})$. Then by 3.1.3(c), (e) and Cartan's fixed point theorem, it follows that the finite group action $H_{1} \times D_{i} \rightarrow D_{i}$ has a nonempty convex totally geodesic submanifold $C_{i} \subset D_{i}$ as fixed point set, where $H_{1}=\left\{\alpha_{1}: \alpha \in H\right\}$. Since $M^{\prime H}$ may be identified with $C_{i}$, it follows that $M^{\prime H}$ is nonempty and contractible. Now we consider the case when $H \in \mathscr{C}$ is a finite group. It follows from Cartan's fixed point theorem that the fixed point set for the finite group action $H \times\left(M \times \mathbf{R}^{a}\right) \rightarrow M \times \mathbf{R}^{a}$ is a nonempty convex totally geodesic submanifold $C \subset M \times \mathbf{R}^{a}$. Moreover, it follows from 
3.1.3 and from Cartan's fixed point theorem that the fixed point set for the finite group action $H \times E \rightarrow E$ is equal to $\bigcup_{i} C_{i} \times S_{i}$, where $C_{i}$ is a convex totally geodesic submanifold of $D_{i}$ and $S_{i}$ is equal to the empty subset of $\mathbf{R}$, all of $\mathbf{R}$, or a point in $\mathbf{R}$ (depending on the value of $i$ ). It follows that $M^{\prime H}$ is contractible.

Since $\Gamma \times M^{\prime} \rightarrow M^{\prime}$ is a universal $(\Gamma, \mathscr{C})$-space, it follows that the quotient of the standard projection $M^{\prime} \times \widehat{Y} \rightarrow M^{\prime}$ under the relevant $\Gamma$-actions-denoted by $\tilde{\rho}: \widetilde{\mathscr{E}}(\xi) \rightarrow \widetilde{B}(\xi)$-is a simplicially stratified fibration which represents the fibered homotopy type of $\rho: \mathscr{E}(\xi) \rightarrow \mathscr{B}(\xi)$. On the other hand, $\bar{\rho}$ is equal to the composite of maps

$$
\overline{\mathscr{E}}(\xi) \stackrel{h}{\rightarrow} \widetilde{\mathscr{E}}(\xi) \stackrel{\tilde{\rho}}{\rightarrow} \widetilde{\mathscr{B}}(\xi),
$$

where $h$ is the quotient of $u \times i d:\left(M \times \mathbf{R}^{a}\right) \times \widehat{Y} \rightarrow M^{\prime} \times \widehat{Y}$ under the relevant $\Gamma$-actions. Note that $h$ is a simplicially stratified fibration having points and lines as fibers. So $\bar{\rho}=\tilde{\rho} \circ h$ is fibered homotopy equivalent to $\tilde{\rho}$ and thus has the fiber homotopy type $\rho(\xi)$.

Finally, recall that $\bar{f}: \overline{\mathscr{E}}(\xi) \rightarrow Y$ is the quotient of the standard projection $\left(M \times \mathbf{R}^{a}\right) \times \widehat{Y} \rightarrow \widehat{Y}$ under the relevant $\Gamma$-actions. Hence, it is a fiber bundle with contractible fiber $M \times \mathbf{R}^{a}$. Consequently, $f$ is a homotopy equivalence.

This completes the proof of Lemma 3.1.2.

Completion of proof of Proposition 2.3. Let $\mathscr{S}_{*}(\mathrm{l})$ denote either of the functors $\mathscr{P}_{*}()$ or $\mathscr{P}_{*}^{\text {diff }}()$. Note, by Lemma 3.1 .2 , that it will suffice to prove that the assembly map $\bar{A}_{*}: \mathbb{H}_{*}\left(\overline{\mathscr{B}}(\xi), \mathscr{S}_{*}(\bar{\rho})\right) \rightarrow \mathscr{S}_{*}(\overline{\mathscr{E}}(\xi))$ for the simplicially stratified fibration $\bar{\rho}: \overline{\mathscr{E}}(\xi) \rightarrow \overline{\mathscr{B}}(\xi)$ is an equivalence of $\Omega$-spectra. We will now state the two lemmas that constitute a proof of this fact.

3.2. Lemma. The assembly map

$$
\mathbb{H}_{*}\left(\overline{\mathscr{B}}(\xi), \mathscr{S}_{*}(\bar{\rho})\right) \stackrel{\bar{A}_{*}}{\rightarrow} \mathscr{S}_{*}(\overline{\mathscr{E}}(\xi))
$$

is surjective on the homotopy group level.

3.3. Lemma. The assembly map

$$
\mathbb{H}_{*}\left(\overline{\mathscr{B}}(\xi), \mathscr{S}_{*}(\bar{\rho})\right) \stackrel{\bar{A}_{*}}{\rightarrow} \mathscr{S}_{*}(\overline{\mathscr{E}}(\xi))
$$

is injective on the homotopy group level.

Before proceeding with the proofs for 3.2 and 3.3 it will be useful to verify the following claim.

3.4. Claim. There is no loss of generality in assuming that $\widehat{Y}$ is a differentiable manifold and that the action $\pi_{1} X: \widehat{Y} \rightarrow \widehat{Y}$ is by diffeomorphisms (cf. 3.1 for $\widehat{Y}$ ).

Towards verifying 3.4 we choose a collection of smooth manifolds $\left\{T_{i}: i \in\right.$ I\} which satisfy the following properties. 
3.5. (a) Each $T_{i}$ is a compact smooth manifold.

(b) $I$ is a directed set. If $i, j \in I$ satisfy $i<j$ then $T_{i}$ is a smooth submanifold of $T_{j}$.

(c) Let $T_{I}$ denote the direct limit space limit $_{i \rightarrow \infty} T_{i}$. Then there is a homotopy equivalence $h: T_{I} \rightarrow Y$.

For each $i \in I$ let $\widehat{T}_{i} \rightarrow T_{i}$ denote the pullback of the covering space projection $\widehat{Y} \rightarrow Y$ along the map $T_{i} \subset T_{I} \stackrel{h}{\rightarrow} Y$. Note that $\pi_{1} X$ acts freely and properly discontinuously on $\widehat{T}_{i}$ with $T_{i}=\widehat{T}_{i} / \pi_{1} X$. Let $\pi_{1} X: M^{\prime} \rightarrow M^{\prime}$ be as in 3.1, and for each $i \in I$ let $\pi_{1} X: M^{\prime} \times \widehat{T}_{i} \rightarrow M^{\prime} \times \widehat{T}_{i}$ denote the diagonal action. Let $\bar{\rho}^{i}: \overline{\mathscr{E}}^{i}(\xi) \rightarrow \overline{\mathscr{B}}^{i}(\xi)$ denote the quotient of the composite map $\left(M \times \mathbf{R}^{a}\right) \times \widehat{T}_{i} \stackrel{u \times \text { id }}{\rightarrow} M^{\prime} \times \widehat{T}_{i} \stackrel{\text { proj }}{\rightarrow} M^{\prime}$ under the $\pi_{1} X$-actions (cf. 3.1 for $\left.u: M \times \mathbf{R}^{a} \rightarrow M^{\prime}\right)$.

It can be argued from 3.5 that the assembly map $\bar{A}_{*}$ of $3.2,3.3$ is equal to the direct limit over $i \in I$ of the assembly maps $\bar{A}_{*}^{i}: \mathbb{H}_{*}\left(\overline{\mathscr{B}}^{i}(\xi), \mathscr{S}_{*}\left(\bar{\rho}^{i}\right)\right) \rightarrow$ $\mathscr{S}_{*}\left(\overline{\mathscr{E}}^{i}(\ddot{\zeta})\right)$ for the stratified fibrations $\bar{\rho}^{i}: \overline{\mathscr{E}}^{i}(\xi) \rightarrow \overline{\mathscr{B}}^{i}(\xi)$.

This completes the verification of 3.4 .

Proof of Lemma 3.2. In order to conserve on notation we will prove Lemma 3.2 for the functor $\mathscr{P}_{*}()$. The same proof works for the functor $\mathscr{P}_{*}^{\text {diff }}()$. We divide the proof into the following three steps. In these steps we abbreviate the notation $\bar{\rho}: \overline{\mathscr{E}}(\xi) \rightarrow \overline{\mathscr{B}}(\xi)$ to $\bar{\rho}: \overline{\mathscr{E}} \rightarrow \overline{\mathscr{B}}$, and set $\Gamma=\pi_{1} X$.

Step 1. In this step we give a geometric formulation of the assembly map in 3.2, 3.3.

From 3.4 it follows that $\overline{\mathscr{E}}$ is a smooth manifold. Thus for any $j \geq 0$ the space $\mathscr{P}_{j}(\overline{\mathscr{E}})$ may be taken to be the space of stable pseudoisotopies of $\overline{\mathscr{E}} \times \mathbf{R}^{j}$ which have compact support in the $\overline{\mathscr{E}}$-factor and which are bounded in the $\mathbf{R}^{j}$-factor; that is, we do not need to refer to the semisimplicial construction for $\mathscr{P}_{j}(\overline{\mathscr{E}})$. For any $\varepsilon>0$ and any stable pseudoisotopy $h: \overline{\mathscr{E}} \times I^{n} \times \mathbf{R}^{j} \times$ $[0,1] \rightarrow \overline{\mathscr{E}} \times I^{n} \times \mathbf{R}^{j} \times[0,1]$ in $\mathscr{P}_{j}(\overline{\mathscr{E}})$ we say that $h$ is $\varepsilon$-controlled over $\overline{\mathscr{B}}$ if for any point $p \in \overline{\mathscr{E}} \times I^{n} \times \mathbf{R}^{j}$ the composite path $[0,1]=p \times[0,1] \stackrel{h}{\rightarrow}$ $\overline{\mathscr{E}} \times I^{n} \times \mathbf{R}^{j} \times[0,1] \stackrel{\text { proj }}{\longrightarrow} \overline{\mathscr{B}}$ has diameter less than or equal to $\varepsilon$ with respect to some fixed metric on $\overline{\mathscr{B}}$. We let $\mathscr{P}_{j}(\overline{\mathscr{E}} ; \bar{\rho})$ denote the space of all continuous maps $f:[0, \infty) \rightarrow \mathscr{P}_{j}(\overline{\mathscr{E}})$ such that $f(t)$ is $(1+t)^{-1}$-controlled over $\overline{\mathscr{B}}$ for all $t \in[0, \infty)$ and such that there is a compact subset $C \subset \overline{\mathscr{E}}$ and a positive number $\alpha>0$ (both of which depend on $f$ ) such that, for each $t \in[0, \infty$ ), both the support of $f(t)$ lies over $C$ and $f(t)$ is $\alpha$-controlled over $\mathbf{R}^{j}$. If $j<0$ then set $\mathscr{P}_{j}(\overline{\mathscr{E}} ; \bar{\rho})=\Omega^{-j} \mathscr{P}_{0}(\overline{\mathscr{E}} ; \bar{\rho})$. Quinn [48] has shown that the collection $\mathscr{P}_{*}(\overline{\mathscr{E}} ; \bar{\rho})=\left\{\mathscr{P}_{j}(\overline{\mathscr{E}} ; \bar{\rho}): j \in Z\right\}$ is an $\Omega$-spectrum and that the following property holds.

3.6. The assembly map $\bar{A}_{*}: \mathbb{H}_{*}\left(\overline{\mathscr{B}}, \mathscr{P}_{*}(\bar{\rho})\right) \rightarrow \mathscr{P}_{*}(\overline{\mathscr{E}})$ is weakly equivalent to the map $i_{*}: \mathscr{P}_{*}(\overline{\mathscr{E}} ; \bar{\rho}) \rightarrow \mathscr{P}_{*}(\overline{\mathscr{E}})$ defined by $i_{j}(f)=f(0)$ for $j \geq 0$. 
Step 2. In this step we review some results from [24] about lifting paths from a manifold into its sphere bundle.

Let $M$ denote a simply connected Riemannian symmetric space with sectional curvature $K \leq 0$ everywhere. Set $N=M \times \mathbf{R}$ equipped with the product Riemannian structure. Each vector $v \in T(N)$ can be written as $v=v_{1} \times v_{2}$ where $v_{1}$ is tangent to $M$ and $v_{2}$ is tangent to $\mathbf{R}$. Let $S N$ denote the unit sphere bundle, and let $S^{+} N$ denote the subbundle of all $v \in S N$ such that $v_{2}=a u$ with $a \geq 0$ where $u: N \rightarrow S N$ is the unit tangent vector field pointing in the direction of increasing $\mathbf{R}$. We have verified the following in [24, §3].

3.7. Given any numbers $\alpha>0, \varepsilon>0$, there are numbers $s>0$ and $\delta>$ 0 . For each path $p:[0,1] \rightarrow M \times[-\delta, \delta]$, which has diameter less than or equal to $\alpha$ in $N$, and for any vector $v \in S^{+} N_{p(0)}$ there is an associated path $p_{v}:[0,1] \rightarrow S^{+} N$ which satisfies the following properties.

(a) $p_{v}$ is a lifting of $p$; that is, the composite map $[0,1] \stackrel{p_{v}}{\rightarrow} S^{+} N \stackrel{\text { proj }}{\rightarrow} N$ is equal to $p$.

(b) Let $p_{v}, p_{w}:[0,1] \rightarrow S^{+} N$ denote two liftings of $p:[0,1] \rightarrow N$. Then $p_{v}(t)=p_{w}(t)$ holds for some $t \in[0,1]$ if and only if $v=w$.

(c) The function which sends $(p, v)$ to $p_{v}$ is continuous in $p$ and $v$.

(d) Let $g^{t}: S^{+} N \rightarrow S^{+} N, t \in \mathbf{R}$, denote the geodesic flow, and let $\mathscr{G}^{+}$ denote the foliation of $S^{+} N$ by the orbits of $g^{t}$. Then any composite path $[0,1] \stackrel{p_{v}}{\rightarrow} S^{+} N \stackrel{g^{s}}{\rightarrow} S^{+} N$ is $(2 \alpha, \varepsilon)$-controlled over $\left(S^{+} N, \mathscr{G}^{+}\right)$; that is, there is a path $q:[0,1] \rightarrow S^{+} N$, which is contained in a segment of a leaf of $\mathscr{G}^{+}$ which has length less than or equal $2 \alpha$, and $d\left(q(t), g^{s} \circ p_{v}(t)\right) \leq \varepsilon$ holds for all $t \in[0,1]$. Here $d($,$) denotes the metric canonically induced on S^{+} N$ by the Riemannian structure on $N$ (cf. [18; part I, 3.4.1]).

(e) Let $r: N \rightarrow N$ denote any isometry of $N$ satisfying $r=\bar{r} \times$ id, where $\bar{r}$ is an isometry of $M$, and let $d r: S^{+} N \rightarrow S^{+} N$ denote the differential of $r$. Then we have that $d r \circ p_{v}=(r \circ p)_{d r(v)}$.

We have indicated the construction of $p_{v}$ in Figure 3.7.1 (p. 272) for the case when $v$ does not lie near $\partial\left(S^{+} N\right)$. If $v$ lies in or near $\partial\left(S^{+} N\right)$ then this construction must be tapered as indicated in [24, §3]. In Figure 3.7.1, y lies a distance $s$ from $p(0)$ along the geodesic $g: \mathbf{R} \rightarrow N$ satisfying $g^{\prime}(0)=v$. If $h: R \rightarrow N$ is the unit speed geodesic going through $p(t)$ at time 0 and going through $y$ at some later time, then $p_{v}(t)=h^{\prime}(0)$ defines the lifting $p_{v}(t)$.

Step 3. In this step we complete the verification of 3.2 by showing that for any map $f: S^{k} \rightarrow \mathscr{P}_{j}(\overline{\mathscr{E}})$-where $k, j$ are positive integers and $S^{k}$ is the $k$ dimensional sphere-there is a homotopy $f_{t}: S^{k} \rightarrow \mathscr{P}_{j}(\overline{\mathscr{E}}), t \in[0,1]$, and there is a map $\bar{f}: S^{k} \rightarrow \mathscr{P}_{j}(\overline{\mathscr{E}} ; \bar{\rho})$, such that $f_{1}=i_{j} \circ \bar{f}$ where $i_{j}$ comes from 3.6.

For each point $x \in S^{k}$ we have a pseudoisotopy $f(x): \overline{\mathscr{E}} \times I^{n} \times \mathbf{R}^{j} \times[0,1] \rightarrow$ $\overline{\mathscr{E}} \times I^{n} \times \mathbf{R}^{j} \times[0,1]$. Since there is the covering space projection $M \times \mathbf{R}^{a} \times$ 

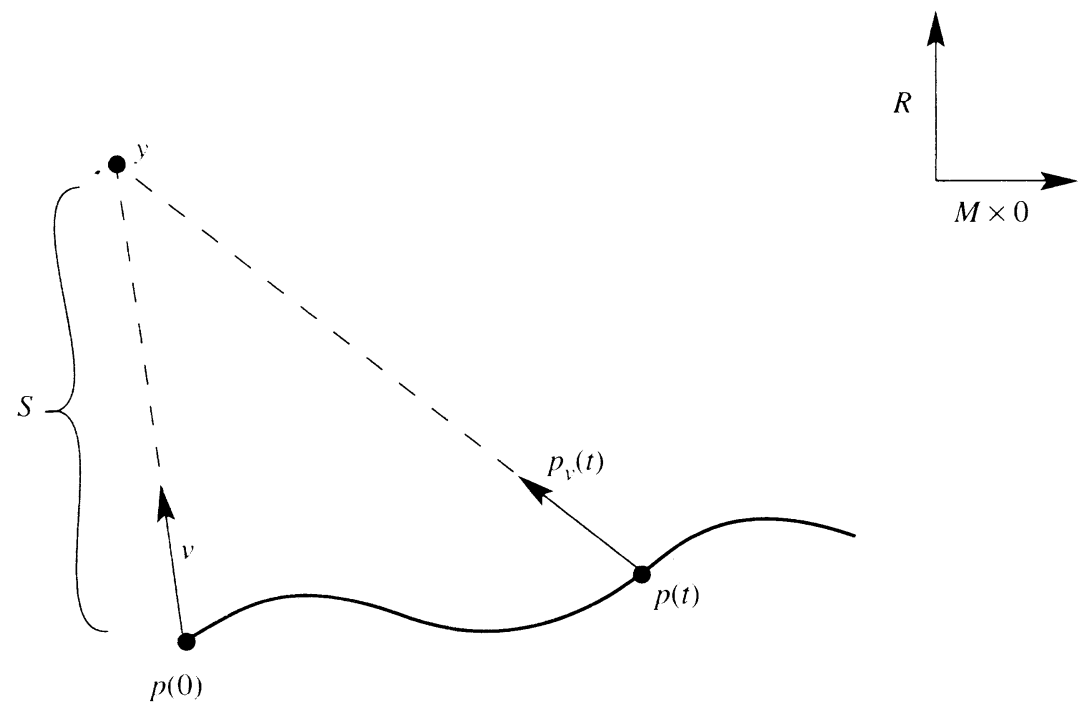

Figure 3.7.1

$\widehat{Y} \rightarrow \overline{\mathscr{E}}=\left(M \times \mathbf{R}^{a} \times \widehat{Y}\right) / \Gamma$, we may lift $f(x)$ uniquely to a pseudoisotopy $\hat{f}(x):\left(M \times \mathbf{R}^{a} \times \widehat{Y}\right) \times I^{n} \times \mathbf{R}^{j} \times[0,1] \rightarrow\left(M \times \mathbf{R}^{a} \times \widehat{Y}\right) \times I^{n} \times \mathbf{R}^{j} \times[0,1]$. Under the inclusion map $M=M \times 0 \subset M \times \mathbf{R}=N$ this becomes a stable pseudoisotopy $\hat{f}(x):\left(N \times \mathbf{R}^{a} \times \widehat{Y}\right) \times I^{n} \times \mathbf{R}^{j} \times[0,1] \rightarrow\left(N \times \mathbf{R}^{a} \times \widehat{Y}\right) \times I^{n} \times \mathbf{R}^{j} \times[0,1]$ and then a stable pseudoisotopy $\widehat{F}(x):\left(S^{+} N \times \mathbf{R}^{a} \times \widehat{Y}\right) \times I^{n} \times \mathbf{R}^{j} \times[0,1] \rightarrow$ $\left(S^{+} N \times \mathbf{R}^{a} \times Y\right) \times I^{n} \times \mathbf{R}^{j} \times[0,1]$ by the formulae of 3.8 .

3.8. For any point $(y, z) \in\left(N, \mathbf{R}^{a} \times \widehat{Y} \times I^{n} \times \mathbf{R}^{j}\right)$ denote the composite path $[0,1]=(y, z) \times[0,1] \stackrel{\hat{f}(x)}{\rightarrow} N \times \mathbf{R}^{a} \times \hat{Y} \times I^{n} \times \mathbf{R}^{j} \times[0,1]$ by $p \times q$, where $p$ is a path in $N$ and $q$ is a path in $\mathbf{R}^{a} \times \widehat{Y} \times I^{n} \times \mathbf{R}^{j} \times[0,1]$. Then for any $v \in S^{+} N_{y}$ define $[0,1]=(v, z) \times[0,1] \stackrel{\widehat{F}(x)}{\rightarrow}\left(S^{+} N \times \mathbf{R}^{a} \times \widehat{Y}\right) \times I^{n} \times \mathbf{R}^{j} \times[0,1]$ to be equal to $p_{v} \times q$, where $p_{v}$ comes from 3.7 .

Note that it follows from $3.7(\mathrm{a})-$ (c) and 3.8 that each $\widehat{F}(x)$ is a well-defined stable pseudoisotopy which is continuous in $x$. Define a one-parameter family $\widehat{F}_{t}(x), t \in[0, s]$, of pseudoisotopies by $\widehat{F}_{t}(x)=\left(g^{t} \times \mathrm{id}\right) \circ \widehat{F}(x) \circ\left(g^{t} \times \mathrm{id}\right)^{-1}$, where id: $\mathbf{R}^{a} \times \widehat{Y} \times I^{n} \times \mathbf{R}^{j} \times[0,1] \rightarrow \mathbf{R}^{a} \times \widehat{Y} \times I^{n} \times \mathbf{R}^{j} \times[0,1]$ is the identity map and $s>0$ comes from 3.7(d). Note that the following holds because of $3.7(\mathrm{~d})$.

3.9. For each $x \in S^{k}$ the pseudoisotopy $\widehat{F}_{s}(x)$ is $(2 \alpha, \varepsilon)$-controlled over $\left(S^{+} N\right.$, $\left.\mathscr{G}^{+}\right)$; that is, for each point $y \in S^{+} N \times \mathbf{R}^{a} \times \widehat{Y} \times I^{n} \times \mathbf{R}^{j}$ the composite path $[0,1]=y \times[0,1] \stackrel{\widehat{F}_{s}(x)}{\rightarrow} S^{+} N \times \mathbf{R}^{a} \times \widehat{Y} \times I^{n} \times \mathbf{R}^{j} \times[0,1] \stackrel{\text { proj }}{\longrightarrow} S^{+} N$ is $(2 \alpha, \varepsilon)-$ controlled over $\left(S^{+} N, \mathscr{G}^{+}\right)$.

We note that all the preceding constructions are invariant under the relevant actions by $\Gamma$ (cf. 3.7(e)) and note that the geodesic flow $g^{t}$ commutes with any 
map $d r: S^{+} N \rightarrow S^{+} N$ from $3.7(\mathrm{e})$. Thus the quotient of each $\widehat{F}_{s}(x)$ under the relevant $\Gamma$-actions is a well-defined pseudoisotopy $F_{s}(x): \tau \times I^{n} \times \mathbf{R}^{j} \times[0,1] \rightarrow$ $\tau \times I^{n} \times \mathbf{R}^{j} \times[0,1]$, where $\tau=\left(S^{+} N \times \mathbf{R}^{a} \times \widehat{Y}\right) / \Gamma$. The standard projection $S^{+} N \times \mathbf{R}^{a} \times \widehat{Y} \rightarrow S^{+} N$ induces a simplicially stratified fibration $\rho: \tau \rightarrow S^{+} N / \Gamma$. Note that $\mathscr{G}^{+} / \Gamma$ is a stratified foliation for $S^{+} N / \Gamma$ in the sense of $[15, \S 1]$. (This follows from the fact that $\Gamma: S^{+} N \rightarrow S^{+} N$ is a properly discontinuous action which permutes the leaves of $\mathscr{G}^{+}$and preserves the direction of the geodesic flow.) We may rephrase 3.9 as follows.

3.10. For each $x \in S^{k}$ the pseudoisotopy $F_{S}(x)$ is $(2 \alpha, \varepsilon)$-controlled over $\left(S^{+} N / \Gamma, \mathscr{G}^{+} / \Gamma\right)$.

Note that 3.10 allows us to apply the fibered-stratified-foliated control theorem $[15,1.11]$ to each $F_{s}(x)$ to get a one-parameter family $F_{t}(x), t \in[s, s+1]$, of stable pseudoisotopies on $\tau$ which satisfy the following properties.

3.11. There is a number $\beta$ which depends only on $\operatorname{dim} N$ and a number $\varepsilon^{\prime}>0$ which depends on $\varepsilon$. Let $C$ denote the subset of $S^{+} N / \Gamma$, which is the union of all leaves of $\mathscr{G}^{+} / \Gamma$ which have length less than or equal to $\beta \alpha$ (with respect to some stratified Riemannian metric on $S^{+} N / \Gamma$; $\mathrm{cf}$. [15, §1]); and, for any $t>0$, let $C_{t}$ denote that part of $C$ which lies over the subset $(M \times[-t, t]) / \Gamma \subset N / \Gamma$; and let $C_{t}^{\varepsilon^{\prime}}$ denote all points in $S^{+} N / \Gamma$ which are a distance less than $\varepsilon^{\prime}$ from $C_{t}$.

(a) For each $x \in S^{k}$ the pseudoisotopy $F_{s+1}(x)$ is $\varepsilon^{\prime}$-controlled over $\left(S^{+} N / \Gamma\right.$ $\left.-C_{\beta S}^{\varepsilon^{\prime}}\right)$ and is $\left(\beta \alpha, \varepsilon^{\prime}\right)$-controlled over $\left(S^{+} N / \Gamma, \mathscr{G}^{+} / \Gamma\right)$.

(b) $\operatorname{limit}_{\varepsilon \rightarrow 0} \varepsilon^{\prime}=0$.

We are essentially done with the proof of Lemma 3.2, we only have to make some identifications to see this. First recall (cf. 3.7(e)) that each $\widehat{F}_{t}(x), t \in$ $[0, s]$, is left invariant under the action of $\Gamma$ on the domain of $\widehat{F}_{t}(x)$; so the quotient of the $\hat{F}_{t}(x), t \in[0, s]$, by the relevant $\Gamma$-action yields the oneparameter family $F_{t}(x), t \in[0, s]$, of stable pseudoisotopies on $\tau$. Note that $\tau$ is a bundle over $\overline{\mathscr{E}}$ having for fiber the space $I^{b} \times \mathbf{R}$ for some integer $b>0$ (to see this note that $S^{+} N$ is a bundle over $M$ having $I^{b} \times \mathbf{R}$ for fiber). Thus we may identify $\mathscr{P}_{*}(\overline{\mathscr{E}})=\mathscr{P}_{*}(\tau)$ via the bundle projection $\tau \rightarrow \overline{\mathscr{E}}$ and identify $F_{t}: S^{k} \rightarrow \mathscr{P}_{*}(\tau), t \in[0, s+1]$, with a homotopy $f_{t}: S^{k} \rightarrow \mathscr{P}_{*}(\overline{\mathscr{E}})$, $t \in[0,1]$, of our original map $f: S^{k} \rightarrow \mathscr{P}_{*}(\overline{\mathscr{E}})$. It remains only to get the map $\bar{f}: S^{k} \rightarrow \mathscr{P}_{*}(\overline{\mathscr{E}} ; \bar{\rho})$ such that $i_{*} \circ \bar{f}=f_{1}$.

To get $\bar{f}^{*}$ it will suffice to identify $\mathscr{P}_{*}(\tau)$ with $\mathscr{P}_{*}(\overline{\mathscr{E}})$ in such a way that 3.11 would translate under this identification into the condition that each $f_{1}(x)$ is $2 \varepsilon^{\prime}$-controlled over $\overline{\mathscr{B}}$ (for then we could apply Quinn's theorem $[48,5.6]$ to $f_{1}$ for sufficiently small $\varepsilon^{\prime}$ to get $\left.\bar{f}\right)$. The desired identification of $\mathscr{P}_{*}(\tau)$ with $\mathscr{P}_{*}(\overline{\mathscr{E}})$ is induced by an embedding $e: \tau \rightarrow \gamma$ (where $\gamma$ is an $m$-disc bundle over $\overline{\mathscr{E}}$ ) constructed as follows. First we use the argument of Remark 
3.1.1.1 to choose an equivariant embedding $e^{\prime}: S^{+} N \times \mathbf{R}^{a} \rightarrow M \times \mathbf{R}^{a} \times I^{m}$, where $I^{m}$ is identified with the unit ball centered at the origin of $\mathbf{R}^{m}$ and where $\Gamma$ acts through a finite quotient group by linear isometries on $I^{m}$ and acts diagonally on $M \times \mathbf{R}^{a} \times I^{m}$. Define $e: \tau \rightarrow \gamma$ to be the quotient under the relevant $\Gamma$-actions of the Cartesian product of the map $e^{\prime}$ with the identity map $\widehat{Y} \rightarrow \widehat{Y}$. We may arrange (cf. Remark 3.1.1.1) that the maps $e, e^{\prime}$ just constructed satisfy the following two properties. (Note that these properties are helpful in establishing the claims at the outset of this paragraph.)

3.12. (a) The composite of $e$ with the bundle projection $\gamma \rightarrow \overline{\mathscr{E}}$ is homotopic to the bundle projection $\tau \rightarrow \overline{\mathscr{E}}$. (See the preceding two paragraphs.)

(b) Let $\pi: S M \rightarrow R P M$ denote the twofold covering projection, and set $E_{i}^{\prime}=\pi^{-1}\left(E_{i}\right)$ (cf. 3.1 for $\left.E_{i}\right)$. For each positive integer $\omega$, set

$$
E^{\omega}=\bigcup_{1 \leq i \leq \omega} E_{i} \text { and } E^{\prime \omega}=\bigcup_{1 \leq i \leq \omega} E_{i}^{\prime} .
$$

Note that $E_{i}^{\prime} \times[-\beta s, \beta s] \subset S(M \times 0) \times[-\beta s, \beta s] \subset S^{+} N$ and that there is a finite integer $\omega$ such that the image of $E^{\prime \omega} \times[-\beta s, \beta s]$ under the covering projection $S^{+} N \stackrel{\text { proj }}{\rightarrow} S^{+} N / \Gamma$ contains the subset $C_{\beta s}$ defined in 3.11 (cf. [24]). Finally recall that we have established embeddings $E_{i} \subset M \times \mathbf{R}^{a}$ in 3.1 .1 . We require that the restricted map $e^{\prime} \mid E^{\prime \omega} \times[-\beta s, \beta s] \times \mathbf{R}^{a}$ be pointwise very close to the composite map

$$
E^{\prime \omega} \times[-\beta s, \beta s] \times \mathbf{R}^{a} \stackrel{\text { proj }}{\rightarrow} E^{\prime \omega} \stackrel{\pi}{\rightarrow} E^{\omega} \subset M \times \mathbf{R}^{a}=M \times \mathbf{R}^{a} \times 0 .
$$

We also require that $e^{\prime}$ be pointwise very close to the composite map

$$
S^{+} N \times \mathbf{R}^{a} \stackrel{\text { proj }}{\rightarrow} S^{+} N \times 0 \stackrel{e^{\prime}}{\rightarrow} M \times \mathbf{R}^{a} \times I^{m} .
$$

This completes the proof of Lemma 3.2.

Proof of Lemma 3.3. In order to conserve on notation we prove Lemma 3.3 for the functor $\mathscr{P}_{*}()$. The same proof works for the functor $\mathscr{P}_{*}^{\text {diff }}()$. We also continue to abbreviate the notation $\bar{\rho}: \overline{\mathscr{E}}(\xi) \rightarrow \overline{\mathscr{B}}(\xi)$ by $\bar{\rho}: \overline{\mathscr{E}} \rightarrow \overline{\mathscr{B}}$, and set $\Gamma=\pi_{1} X$.

The strategy of this proof is to define a map $r_{*}: \mathscr{P}_{*}(\overline{\mathscr{E}}) \rightarrow \mathscr{S}_{*}$ between $\Omega$ spectra (where $\mathscr{S}_{*}$ will be introduced later) and then to show that the composite map $r_{*} \circ \bar{A}_{*}: \mathbb{H}_{*}\left(\overline{\mathscr{B}}, \mathscr{P}_{*}(\bar{\rho})\right) \rightarrow \mathscr{S}_{*}$ is a weak equivalence between $\Omega$-spectra. This will be carried out in the following four steps.

Step 1. In this step we define the map $r_{*}$.

We will use the shorter notation $\hat{\mathscr{E}} \rightarrow \overline{\mathscr{E}}$ to denote the covering projection $M \times \mathbf{R}^{a} \times \widehat{Y} \rightarrow M \times \mathbf{R}^{a} \times \widehat{Y} / \Gamma=\overline{\mathscr{E}}$. Let $\Gamma \times M^{\prime} \rightarrow M^{\prime}$ be as in 3.1 , and let $\Gamma \times M^{\prime} \times \hat{\mathscr{E}} \rightarrow M^{\prime} \times \hat{\mathscr{E}}$ denote the diagonal action. We define the maps

$$
\gamma: \mathscr{D} \rightarrow \mathscr{D}^{\prime}, \quad \tau: \mathscr{D}^{\prime} \rightarrow \overline{\mathscr{B}}
$$

as follows. Let $\mathscr{D}$ denote the quotient of $M^{\prime} \times \widehat{\mathscr{E}}$ under the diagonal action of $\Gamma$. For each $y \in M^{\prime}$, let $\Gamma^{y}$ denote the isotropy group at the point $y$ for 
the action $\Gamma \times M^{\prime} \rightarrow M^{\prime}$. When $\Gamma^{y}$ is an infinite group, let $E_{i_{y}}$ denote the path component of $E$ such that some element of infinite order in $\Gamma^{y}$ leaves invariant each leaf of the foliation $F_{i_{y}}$ (cf. 3.1.3). Let $q: M^{\prime} \times\left(M \times \mathbf{R}^{a}\right) \rightarrow Q$ denote the quotient map obtained by collapsing each leaf of $y \times F_{i_{y}}, y \in M^{\prime}$, with $\Gamma^{y}$ infinite, to a point; note that the action of $\Gamma$ on $M^{\prime} \times\left(M \times \mathbf{R}^{a}\right)$ induces an action of $\Gamma$ on $Q$. Define $\gamma: \mathscr{D} \rightarrow \mathscr{D}^{\prime}$ to be the quotient of the composite map $M^{\prime} \times \widehat{\mathscr{E}}=M^{\prime} \times\left(M \times \mathbf{R}^{a} \times \widehat{Y}\right) \stackrel{\text { proj }}{\rightarrow} M^{\prime} \times\left(M \times \mathbf{R}^{a}\right) \stackrel{q}{\rightarrow} Q$ under the relevant $\Gamma$-actions. Note that the standard projection $M^{\prime} \times\left(M \times \mathbf{R}^{a}\right) \rightarrow M^{\prime}$ induces a $\operatorname{map} \tau: \mathscr{D}^{\prime} \rightarrow \overline{\mathscr{B}}$.

For each $b \in \overline{\mathscr{B}}$ we denote the fiber $\tau^{-1}(b)$ by $\mathscr{D}_{b}^{\prime}$. We equip each $\mathscr{D}_{b}^{\prime}$ with a metric

$$
d_{b}(,)
$$

described as follows. Choose $y \in M^{\prime}$, which is mapped to $b$ by the projection $M^{\prime} \rightarrow \overline{\mathscr{B}}(\xi)=M^{\prime} / \Gamma$, and let $\Gamma^{y}$ be the isotropy group at the point $y \in M^{\prime}$ for the action $\Gamma \times M^{\prime} \rightarrow M^{\prime}$. By the definition of $\gamma: \mathscr{D} \rightarrow \mathscr{D}^{\prime}$ there is a canonical quotient map $q_{y}: M \times \mathbf{R}^{a} \rightarrow \mathscr{D}_{b}^{\prime}$ which factors through $M \times \mathbf{R}^{a} / \Gamma^{y}$. Let $d_{b}($,$) denote the metric on \mathscr{D}_{b}^{\prime}$ induced from the given Riemannian metric on $M \times \mathbf{R}^{a}$. (Recall that $M \times \mathbf{R}^{a}$ is equipped with the product metric of the Riemannian metric on $M$ and the Euclidean metric on $\mathbf{R}^{a}$. Let $d($, denote the induced metric on $M \times \mathbf{R}^{a}$.) That is, for $u, v \in \mathscr{D}_{b}^{\prime}$, set

$$
d_{b}(u, v)=\text { infinum }\left\{\Sigma_{i} d\left(x_{i}, z_{i+1}\right)\right\},
$$

where $x_{0}, x_{1}, \ldots, x_{n-1}$ and $z_{1}, z_{2}, \ldots, z_{n}$ are any finite sequences of points in $M \times \mathbf{R}^{a}$ such that $q_{y}\left(x_{0}\right)=u, q_{y}\left(z_{n}\right)=v$, and $q_{y}\left(x_{i}\right)=q_{y}\left(z_{i}\right)$ for $i=$ $1,2, \ldots, n-1$.

For each $b \in \overline{\mathscr{B}}$ we define a point

$$
S_{b} \in \mathscr{D}_{b}^{\prime}
$$

as follows. Define $\eta: M \times \mathbf{R}^{a} / \Gamma \rightarrow \mathscr{D}^{\prime}$ to be the quotient under the relevant $\Gamma$-actions of the composite of $q: M^{\prime} \times\left(M \times \mathbf{R}^{a}\right) \rightarrow Q$ with the product map $M \times \mathbf{R}^{a} \stackrel{u \times \text { id }}{\rightarrow} M^{\prime} \times\left(M \times \mathbf{R}^{a}\right)$, where $u: M \times \mathbf{R}^{a} \rightarrow M^{\prime}$ comes from 3.1 and id : $M \times R^{a} \rightarrow M \times \mathbf{R}^{a}$ is the identity map. Set $S_{b}=\operatorname{Image}(\eta) \cap \mathscr{D}_{b}^{\prime}$.

Even though $\mathscr{D}$ is not a manifold (except when $M$ is a point) we may define spaces of stable "pseudoisotopies" of $\mathscr{D}, \mathscr{P}_{j}(\mathscr{D})$, by appealing to the constructions of the first paragraph of 1.1. Thus if $j \geq 0$ then a point in $\mathscr{P}_{j}(\mathscr{D})$ is represented by a homeomorphism $h: \mathscr{D} \times I^{n} \times \mathbf{R}^{j} \times[0,1] \rightarrow \mathscr{D} \times I^{n} \times \mathbf{R}^{j} \times$ $[0,1]$, which is bounded in the $\mathbf{R}^{j}$-factor and such that the restricted map $h \mid\left(\mathscr{D} \times I^{n} \times \mathbf{R}^{j} \times 0\right)$ is the inclusion. (Note that we do not require $h$ to have compact support in the factor $\mathscr{D}$.) For $j<0$ we set $\mathscr{P}_{j}(\mathscr{D})=\Omega^{-j} \mathscr{P}_{0}(\mathscr{D})$.

For each integer $j \geq 0$, we define

$$
\mathscr{P}_{j}(\mathscr{D} ; \gamma, \tau)
$$


to be the space of all mappings $g:[0, \infty) \rightarrow \mathscr{P}_{j}(\mathscr{D})$ which satisfy the following properties, where $\bar{\gamma}: \mathscr{D} \rightarrow \overline{\mathscr{B}}$ is the composite $\tau \circ \gamma$. (Note that $\bar{\gamma}$ is the quotient of the projection $M^{\prime} \times \widehat{\mathscr{E}} \rightarrow M^{\prime}$ under the relevant $\Gamma$-actions.)

3.17. (a) The bounds for each $g(t)$ in the $\mathbf{R}^{j}$-factor is independent of $t$.

(b) For each $b \in \overline{\mathscr{B}}$, we let $\mathscr{D}_{b}$ denote the subspace $\bar{\gamma}^{-1}(b) \subset \mathscr{D}$, and we require that $h\left(\mathscr{D}_{b} \times I^{n} \times \mathbf{R}^{j} \times[0,1]\right) \subset \mathscr{D}_{b} \times I^{n} \times \mathbf{R}^{j} \times[0,1]$ holds for all $b$.

(c) There is a number $\alpha \geq 1$ which depends on $g$. For all $t \in[0, \infty)$ and for all $b \in \overline{\mathscr{B}}$ the stable pseudoisotopy $g(t): \mathscr{D}_{b} \times I^{n} \times \mathbf{R}^{j} \times[0,1] \rightarrow$ $\mathscr{D}_{b} \times I^{n} \times \mathbf{R}^{j} \times[0,1]$ is $\alpha(1+t)^{-1}$-controlled over $\mathscr{D}_{b}^{\prime}$ (with respect to the projection $\gamma: \mathscr{D}_{b} \rightarrow \mathscr{D}_{b}^{\prime}$ and the metric $\left.d_{b}(),\right)$.

(d) Given any compact subset $C \subset \mathscr{D}^{\prime}$, there exists a compact subset $B \subset \mathscr{D}$ such that

$$
\left(\gamma^{-1}(C) \times I^{n} \times \mathbf{R}^{j} \times[0,1]\right) \cap \operatorname{support}(g(t)) \subset B \times I^{n} \times \mathbf{R}^{j} \times[0,1]
$$

holds for all $t \in[0, \infty)$.

(e) Mioreover, there is a compact subset $A \subset \overline{\mathscr{B}}$ such that

$$
\operatorname{support}(g(t)) \subset \bar{\gamma}^{-1}(A) \times I^{n} \times \mathbf{R}^{j} \times[0,1]
$$

holds for all $t \in[0, \infty)$.

Quinn's argument [48] can be adapted to prove that there is an equivalence of $\Omega$-spectra $\mathscr{P}_{i}(\mathscr{D} ; \gamma, \tau) \cong \Omega \mathscr{P}_{i+1}(\mathscr{D} ; \gamma, \tau)$ for all integers $i \geq 0$ (cf. [24, $\S 5])$. Thus the collection $\mathscr{S}_{*}=\left\{\mathscr{S}_{i}: i \in \mathbf{Z}\right\}$, defined by $\mathscr{S}_{i}=\mathscr{P}_{i}(\mathscr{D} ; \gamma, \tau)$ if $i \geq 0$ and by $\mathscr{S}_{i}=\Omega^{-i} \mathscr{P}_{0}(\mathscr{D} ; \gamma, \tau)$ if $i<0$, is an $\Omega$-spectrum.

The map of $\Omega$-spectra

$$
r_{*}: \mathscr{P}_{*}(\overline{\mathscr{E}}) \rightarrow \mathscr{S}_{*}
$$

is defined as follows. For integer $j \geq 0$ let $h: \overline{\mathscr{E}} \times I^{n} \times \mathbf{R}^{j} \times[0,1] \rightarrow \overline{\mathscr{E}} \times I^{n} \times \mathbf{R}^{j} \times$ $[0,1]$ represent a point in $\mathscr{P}_{j}(\overline{\mathscr{E}})$. Pull $h$ back along the covering projection $\widehat{\mathscr{E}} \rightarrow \overline{\mathscr{E}}$ to get a pseudoisotopy $\hat{h}: \hat{\mathscr{E}} \times I^{n} \times \mathbf{R}^{j} \times[0,1] \rightarrow \widehat{\mathscr{E}} \times I^{n} \times \mathbf{R}^{j} \times[0,1]$. Define $h^{\prime}: \mathscr{D} \times I^{n} \times \mathbf{R}^{j} \times[0,1] \rightarrow \mathscr{D} \times I^{n} \times \mathbf{R}^{j} \times[0,1]$ to be the quotient of the pseudoisotopy id $\times \hat{h}: M^{\prime} \times\left(\hat{\mathscr{E}} \times I^{n} \times \mathbf{R}^{j} \times[0,1]\right) \rightarrow M^{\prime} \times\left(\hat{\mathscr{E}} \times I^{n} \times \mathbf{R}^{j} \times[0,1]\right)$ under the relevant $\Gamma$-actions, where id: $M^{\prime} \rightarrow M^{\prime}$ denotes the identity map. Now we define $r_{j}(h)$ to be the point of $\mathscr{P}_{j}(\mathscr{D} ; \gamma, \tau)$ represented by the map $g^{\prime}:[0, \infty) \rightarrow \mathscr{P}_{j}(\mathscr{D})$, where $g^{\prime}(t)=g(t+1)$ and $g$ is given in the following claim.

3.19. Claim. There is a mapping $g:[0, \infty) \rightarrow \mathscr{P}_{j}(\mathscr{D})$, which satisfies the following properties.

(a) $g(0)=h^{\prime}$.

(b) $g^{\prime}:[0, \infty) \rightarrow \mathscr{P}_{j}(\mathscr{D})$ represents a point of $\mathscr{P}_{j}(\mathscr{D} ; \gamma, \tau)$, where $g^{\prime}(t)=$ $g(t+1)$; i.e., $g^{\prime}$ satisfies $3.17(\mathrm{a})-(\mathrm{e})$.

(c) There is a compact subset $C \subset \overline{\mathscr{B}}$ and a number $\varepsilon>0$ such that for each $b \in(\overline{\mathscr{B}}-C)$ and for each $t \in[0, \infty)$ we have that the restricted 
map $g(t) \mid\left(\gamma^{-1}\left(S_{b}^{\varepsilon}\right) \times I^{n} \times \mathbf{R}^{j} \times[0,1]\right)$ is the inclusion, where $S_{b}^{\varepsilon}=\{x \in$ $\left.\mathscr{D}_{b}^{\prime}: d_{b}\left(x, S_{b}\right) \leq \varepsilon\right\}$. (Compare with $3.17(\mathrm{e})$.)

(d) $g$ satisfies $3.17(\mathrm{a})-(\mathrm{d})$.

Before proving 3.19 it is necessary to introduce three new maps, namely, $\gamma^{\prime}: \mathscr{D} \rightarrow \mathscr{D}^{\prime \prime}, \tau^{\prime}: \mathscr{D}^{\prime \prime} \rightarrow \overline{\mathscr{B}}, \kappa: \mathscr{D} \rightarrow \mathscr{D}^{\prime \prime}$, and a metric $d_{b}^{\prime}($,$) on each$ fiber $\mathscr{D}_{b}^{\prime \prime}=\tau^{\prime-1}(b), b \in \overline{\mathscr{B}}$, of the simplicially stratified fibration $\tau^{\prime}$. For each $y \in M^{\prime}$ such that $\Gamma^{y}$ is infinite we let $E_{i_{y}} \subset M \times \mathbf{R}^{a}, F_{i_{y}}$ be as described just after 3.13 above, and we let $E_{i_{y}}^{\prime} \subset M, F_{i_{y}}^{\prime}$ denote the images of $E_{i_{y}}, F_{i_{y}}$ under the standard projection $M \times \mathbf{R}^{a} \rightarrow M$. Now by using $E_{i_{y}}^{\prime} \times 0 \subset M \times \mathbf{R}^{a}$ and $F_{i_{y}}^{\prime} \times 0$ in place of $E_{i_{y}} \subset M \times \mathbf{R}^{a}$ and $F_{i_{y}}$ in the construction proceeding 3.13 we get the maps $\gamma^{\prime}, \tau^{\prime}$ (in place of the maps $\gamma, \tau$ ). The metrics $\left\{d_{b}^{\prime}():, b \in\right.$ $\overline{\mathscr{B}}\}$ are constructed as in 3.14 (using $\gamma^{\prime}, \tau^{\prime}$ in place of $\gamma, \tau$ ). Topologically there is no difference between the pairs of maps $(\gamma, \tau)$ and $\left(\gamma^{\prime}, \tau^{\prime}\right)$; however, the metrics $\left\{d_{b}^{\prime}():, b \in \overline{\mathscr{B}}\right\}$ are much better behaved than the metrics $\left\{d_{b}():, b \in \overline{\mathscr{B}}\right\}$. For example, on each stratum $X_{i, b}$ of each fiber $\mathscr{D}_{b}^{\prime \prime}$, $b \in \overline{\mathscr{B}}$, the metric $d_{b}^{\prime}($,$) agrees locally with a Riemannian metric \langle,\rangle_{i, b}$ which has nonpositive sectional curvature values everywhere (and this is not the case for $\left.d_{b}(),\right)$. We shall say that $\left(\mathscr{D}_{b}^{\prime \prime}, d_{b}^{\prime}(),\right)$ has radius of injectivity greater than $\alpha>0$ if in each stratum $X_{i, b}$ any two $\langle,\rangle_{i, b}$-geodesics of length less than or equal to $\alpha$, which start and end at the same two points of $X_{i, b}$, must be equal up to reparametrization. Although the metrics $d_{b}(),, d_{b}^{\prime}($, have different local properties, globally they are quite similar. In fact, there is a number $\lambda>1$ and a homeomorphism $\kappa: \mathscr{D}^{\prime} \rightarrow \mathscr{D}^{\prime \prime}$ which satisfy the following properties: $\kappa\left(\mathscr{D}_{b}^{\prime}\right)=\mathscr{D}_{b}^{\prime \prime}$ for all $b \in \overline{\mathscr{B}}$; and $\lambda^{-1} d_{b}^{\prime}(\kappa(x), \kappa(y)) \leq$ $d_{b}(x, y) \leq \lambda d_{b}^{\prime}(\kappa(x), \kappa(y))$ for all $b \in \overline{\mathscr{B}}$ and for all $x, y \in \mathscr{D}_{b}^{\prime}$.

We can now complete the proof of Claim 3.19. If $C$ is chosen large enough in $3.19(\mathrm{c})$, then for every $b \in(\overline{\mathscr{B}}-C)$ we will have that $h^{\prime} \mid\left(\gamma^{-1}\left(S_{b}^{\beta}\right) \times I^{n} \times\right.$ $\left.\mathbf{R}^{j} \times[0,1]\right)=$ inclusion, $\mathscr{D}_{b}^{\prime \prime}$ has radius of injectivity greater than $\beta$, and $h^{\prime} \mid\left(\mathscr{D}_{b} \times I^{n} \times R^{j} \times[0,1]\right)$ is $\sigma$-controlled over $\left(\mathscr{D}_{b}^{\prime \prime}, d_{b}^{\prime}(),\right)$ (with respect to the map $\mathscr{D}_{b} \stackrel{\gamma}{\rightarrow} \mathscr{D}_{b}^{\prime} \stackrel{\kappa}{\rightarrow} \mathscr{D}_{b}^{\prime \prime}$ ), where $\sigma, \beta>0$ are numbers such that $\lambda^{-1} \sigma^{-1} \beta>>1$. Thus we may apply a stratified fibered version of [24, 3.17] to get $g(t) \mid\left(\mathscr{D}_{b} \times\right.$ $\left.I^{n} \times \mathbf{R}^{j} \times[0,1]\right), t \in[0, \infty)$, which satisfies $3.19(\mathrm{a}),(\mathrm{c}),(\mathrm{d})$. Now, after pushing the support of $g(t) \mid\left(\mathscr{D}_{b} \times I^{n} \times \mathbf{R}^{j} \times[0,1]\right), t \in\left[\frac{1}{2}, \infty\right)$, to infinity, we may reparameterize $g()$ over $\mathscr{D}_{b}^{\prime}$ so that $3.19(\mathrm{~b})$ is also satisfied. To get $g(t) \mid\left(\mathscr{D}_{b} \times I^{n} \times \mathbf{R}^{j} \times[0,1]\right), t \in[0, \infty), b \in C$, we first use fiberedequivariant versions of the geometric constructions of $[24,4.11 .1]$ to obtain $g(t) \mid\left(\mathscr{D}_{b} \times I^{n} \times \mathbf{R}^{j} \times[0,1]\right), t \in[0,1]$, such that $g(1) \mid\left(\mathscr{D}_{b} \times I^{n} \times \mathbf{R}^{j} \times[0,1]\right)$ is $\varepsilon$-controlled over $\left(\mathscr{D}_{b}^{\prime \prime}, d_{b}^{\prime}(),\right)$, where $\varepsilon$ is much smaller than the radius of injectivity for $\left(\mathscr{D}_{b}^{\prime \prime}, d_{b}^{\prime}(),\right)$. Next we apply a stratified fibered version of $[24,3.17]$ to get $g(t) \mid\left(\mathscr{D}_{b} \times I^{n} \times \mathbf{R}^{j} \times[0,1]\right), t \in[1, \infty)$. For more details the reader is referred to [24]. 
Step 2. In this step we define two homology functors $\mathbb{H}_{*}^{1}\left(\right.$, ) and $\mathbb{H}_{*}^{2}($, $)$ whose domains may be any "admissible pair" in $\overline{\mathscr{B}}$.

Let $K$ denote the triangulation of $\overline{\mathscr{B}}$ given by Lemma 3.1.2. (That is, $\bar{\rho}$ is a simplicially stratified fibration with respect to $K$, and $K$ is a triangulation of the smoothly stratified space $\left.\overline{\mathscr{B}}=M^{\prime} / \Gamma\right)$. By a codimension $k$ submanifold pair $(T, \partial T)$ of $\overline{\mathscr{B}}$ (where $k \geq 0$ is an integer) we mean a pair of subsets $T, \partial T$ of $K$, satisfying: each of $T$ and $\partial T$ is a closed PL subset of $\overline{\mathscr{B}}$; for each simplex $\Delta \in K$ the pair $(T, \partial T) \cap \Delta$ is a codimension $k$ PL submanifold pair of $\Delta$ which intersects each face of $\Delta$ transversely. A pair of subsets $(A, B)$ of $\overline{\mathscr{B}}$ is called an admissible pair if the following properties hold: $A$ and $B$ are compact subsets of $\overline{\mathscr{B}} ; B \subset A ;(A, \partial A),(B, \partial B)$, and $(Z=$ closure $(A-B), \partial Z)$ are all codimension zero submanifold pairs of $\overline{\mathscr{B}}$, where the manifold boundaries coincide with the topological boundaries in $\overline{\mathscr{B}}$; each of $\left(\partial_{1} Z, \Lambda Z\right),\left(\partial_{2} Z, \Lambda Z\right)$ is a codimension one submanifold pair of $\overline{\mathscr{B}}$, where $\partial_{1} Z=B \cap Z, \partial_{2} Z=\operatorname{closure}\left(\partial Z-\partial_{1} Z\right)$, and $\Lambda Z=\partial_{1} Z \cap \partial_{2} Z$.

For any admissible pair $(A, B)$ we set

$$
\mathbb{H}_{*}^{1}(A, B)=\mathscr{P}_{*}\left(\bar{\rho}_{A-B}\right),
$$

where $\bar{\rho}_{A-B}=\bar{\rho} \mid \bar{\rho}^{-1}(A-B)$; where $\mathscr{P}_{i}\left(\bar{\rho}_{A-B}\right)$, for each integer $i \geq 0$, denotes the space of all maps $f:[0, \infty) \rightarrow \mathscr{P}_{i}\left(\bar{\rho}^{-1}(A-B)\right)$ such that for all $t \in[0, \infty)$ the stable pseudoisotopy $f(t)$ is $(1+t)^{-1}$-controlled over the subset $A-B$ of $\overline{\mathscr{B}}$. Here $\mathscr{P}_{i}\left(\bar{\rho}^{-1}(A-B)\right)$ denotes the space of all stable pseudoisotopies of $\bar{\rho}^{-1}(A-B) \times \mathbf{R}^{i}$ which are bounded in the $\mathbf{R}^{i}$-factor, and whose support (denoted by $S$ ) satisfies the following: for each compact $C \subset A-B$ there is a compact $D \subset \bar{\rho}^{-1}(A-B)$ with $S \cap \bar{\rho}^{-1}(C) \times \mathbf{R}^{i} \subset D \times \mathbf{R}^{i}$. (Note we do not require $S \subset D \times \mathbf{R}^{i}$ for some compact $D \subset \bar{\rho}^{-1}(A-B)$ unless $A-B$ is compact.)

We also set

$$
\mathbb{H}_{*}^{2}(A, B)=\mathscr{P}_{*}\left(\mathscr{D}_{A-B}, \mathscr{D}_{\partial_{1} Z} ; \gamma, \tau\right)
$$

where $\mathscr{D}_{S}=\bar{\gamma}^{-1}(S)$ for any subset $S \subset \overline{\mathscr{B}}$, and where $\mathscr{P}_{j}\left(\mathscr{D}_{A-B}, \mathscr{D}_{\partial_{1} Z} ; \gamma, \tau\right)$, for each integer $j \geq 0$, denotes the set of all maps $g:[0, \infty) \rightarrow \mathscr{P}_{j}\left(\mathscr{D}_{A-B}\right)$ which satisfy the following properties: $g(t) \mid\left(\mathscr{D}_{\partial_{2} Z-\Lambda Z} \times I^{n} \times \mathbf{R}^{j} \times[0,1]\right)$ is the inclusion map for all $t \in[0, \infty)$; and $g$ satisfies $3.17(\mathrm{a})-(\mathrm{d})$, where in $3.17(\mathrm{~d})$ we require that $C \subset \tau^{-1}(A-B)$, and in $3.17(\mathrm{~b})$, (c) we require that $b \in A-B$.

If $B$ is the empty set then we set $\mathbb{H}_{*}^{i}(A)=\mathbb{H}_{*}^{i}(A, B)$ for $i=1,2$.

We note that the functors $\mathbb{H}_{*}^{1}($,$) and \mathbb{H}_{*}^{2}($,$) are \Omega$-spectra valued (cf. $[24, \S 5 ; 48]$ ), and that they satisfy the usual axioms of homology theory. (This last fact is a deep result proven by Quinn in [48] for $\mathbb{H}_{*}^{1}($,$) and is an easy$ exercise for $\mathbb{H}_{*}^{2}($, , ).

Step 3. We claim that there is a natural (up to homotopy) map $r(,)_{*}$ : $\mathbb{H}_{*}^{1}(,) \rightarrow \mathbb{H}_{*}^{2}(, \quad)$ between homology functors, such that if $A_{1} \subset A_{2} \subset \cdots$ 
is an increasing sequence of compact codimension zero "submanifolds" of $\overline{\mathscr{B}}$ with $\bigcup_{i} A_{i}=\overline{\mathscr{B}}$, then $r_{*} \circ \bar{A}_{*}$ is homotopic to $\operatorname{limit}_{i \rightarrow \infty} r\left(A_{i}, \phi\right)$, where $r_{*}$ comes from 3.18. (Here we identify the assembly map $\bar{A}_{*}$ with the map $i_{*}$ of 3.6.) The proof of this consists of a fibered and equivariant version of arguments given in [24, 6.8]. We refer the reader to [24] for more details.

If $B=\phi$ then we set $r(A)_{*}=r(A, B)_{*}$.

Step 4. In this step we complete the proof of Lemma 3.3.

Let $K$ denote the triangulation for $\mathscr{\mathscr { B }}$ used in Step 2 above. Let $K^{*}$ denote the "dual cell structure" for $K$; that is, for each simplex $\Delta \in K$ the "dual cell" $\Delta^{*} \in K^{*}$ is the union of all simplices $e \in K^{(1)}$ in the first barycentric subdivision of $K$ such that $e \cap \Delta=b(\Delta)$, where $b(\Delta)$ denotes the barycenter of $\Delta$. The "dual cells" $\Delta^{*} \in K^{*}$ are always cone spaces, but they are not always PL cells because $\overline{\mathscr{B}}$ is not in general a PL manifold. Let $K_{1}^{*} \subset K_{2}^{*} \subset K_{3}^{*} \subset \cdots$ denote a finite increasing sequence of subcomplexes of $K^{*}$ such that for all $1 \leq i<\infty$ we have that $K_{i+1}^{*}=K_{i}^{*} \cup \Delta_{i}^{*}$ for some $\Delta_{i}^{*} \in K^{*}$ with $\operatorname{dim} \Delta_{i}^{*} \geq \operatorname{dim} K_{i}^{*}$, with $\partial \Delta_{i}^{*} \subset K_{i}^{*}$, and such that $\bigcup_{i} K_{i}^{*}=A$, where $A$ is an arbitrarily large compact codimension zero "submanifold" of $\overline{\mathscr{B}}$. Define an increasing sequence of subsets $S_{1} \subset S_{2} \subset S_{3} \subset \cdots$ of $\overline{\mathscr{B}}$ by induction as follows: $S_{i+1}$ is the union of $S_{i}$ with all simplices $e \in K^{(i+2)}$ in the $(i+2)$-fold barycentric subdivision of $K$ which intersect with $K_{i+1}^{*}$. Note that each $S_{i}$ is a "regular neighborhood" for $K_{i}^{*}$ in $\overline{\mathscr{B}}$ and that each pair $\left(S_{i+1}, S_{i}\right)$ is an admissible pair.

To complete the proof of 3.3 we proceed by induction over the sequences $S_{1} \subset S_{2} \subset S_{3} \cdots$. Our ( $t$ th step) induction hypothesis is that the map $r\left(S_{t}\right)_{*}$ : $\mathbb{H}_{*}^{1}\left(S_{t}\right) \rightarrow \mathbb{H}_{*}^{2}\left(S_{t}\right)$ is a weak equivalence of $\Omega$-spectra. To carry out the induction step we consider the homotopy commutative diagram

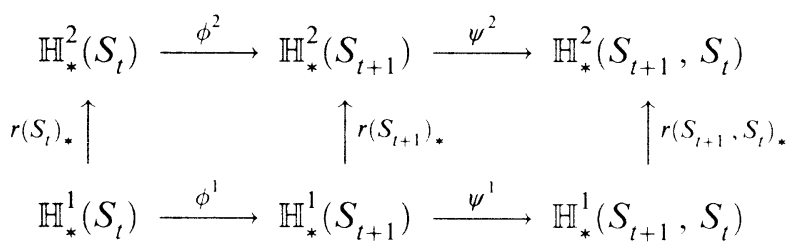

The horizontal maps in this diagram, which are induced by the inclusion maps $S_{t} \subset S_{t+1}$ and $\left(S_{t+1}, \phi\right) \subset\left(S_{t+1}, S_{t}\right)$, are fibrations in the category of $\Omega$-spectra (this is one of the homology axioms for $\mathbb{H}_{*}^{1}($,$) and \mathbb{H}_{*}^{2}($,$) ). Thus to complete$ the induction step it will suffice to show that $r\left(S_{t+1}, S_{t}\right)_{*}$ is a weak equivalence of $\Omega$-spectra. Roughly speaking the map $r\left(S_{t+1}, S_{t}\right)_{*}$ can be shown to be the $u$ fold loop of the map $r\left(\overline{\mathscr{B}}, B_{t}\right)_{*}$, where $A_{t}$ is a regular neighborhood in $\overline{\mathscr{B}}$ for the barycenter of $\Delta_{t}, B_{t}=\operatorname{closure}\left(\overline{\mathscr{B}}-A_{t}\right)$, and $u=\operatorname{dim} \Delta_{t}$, and $r\left(\overline{\mathscr{B}}, B_{t}\right)_{*}$ can easily be seen to be an equivalence. The details of this induction argument consist of fibered and equivariant versions of the arguments given in [24, proof of 6.8.3]. For more details the reader is referred to [24].

This completes the proof of Lemma 3.3. 


\section{Proof of Proposition 2.4}

We will first prove 2.4 in the special case that $\pi_{1} X$ is a finitely generated virtually nilpotent group. (Note that every finitely generated nilpotent group is a virtually poly- $Z$ group.) The proof depends on the following lemma, which is proven at the end of this section. For any virtually poly- $Z$ group $\Gamma$ we denote by $\operatorname{vcd}(\Gamma)$ the virtual cohomological dimension of $\Gamma$.

4.1. Lemma. Suppose that $\Gamma$ is a finitely generated virtually nilpotent group with $\operatorname{vcd}(\Gamma)=k<\infty$. Then there is a short exact sequence of groups

$$
1 \rightarrow \Gamma^{\prime} \rightarrow \Gamma \rightarrow A \rightarrow 1,
$$

where $\Gamma^{\prime}$, A satisfy the following properties:

(a) $\Gamma^{\prime}$ is either finite or is a finitely generated virtually nilpotent group with $\operatorname{vcd}\left(\Gamma^{\prime}\right) \leq k-2$.

(b) There is a short exact sequence of groups

$$
1 \rightarrow \mathbf{Z}^{n} \rightarrow A \rightarrow F \rightarrow 1
$$

where $F$ is finite and the representation $F \rightarrow \operatorname{Aut}\left(\mathbf{Z}^{n}\right)$ given by conjugation is faithful.

Proof of Proposition 2.4 (for finitely generated virtually nilpotent groups). The proof proceeds by induction over the value of $\operatorname{vcd}\left(\pi_{1}(X)\right)$. Suppose that we have already shown that for any space $Y$, such that $\pi_{1} Y$ is virtually nilpotent with $\operatorname{vcd}\left(\pi_{1} Y\right) \leq r$, the Fibered Conjecture 1.7 is true for the functors $\mathscr{P}_{*}()$ and $\mathscr{P}_{*}^{\text {diff }}()$. Now let $X$ denote a space such that $\pi_{1} X$ is virtually nilpotent with $\operatorname{vcd}\left(\pi_{1} X\right)=r+1$. Applying 4.1 to $\pi_{1} X$ we get a short exact sequence.

$$
1 \rightarrow \Gamma^{\prime} \rightarrow \pi_{1} X \rightarrow A \rightarrow 1
$$

with $\Gamma^{\prime}, A$ satisfying $4.1(\mathrm{a})$, (b). The fundamental results of Bieberbach (cf. [56]) and Zassenhaus [59] show that condition 4.1(b) characterizes crystallographic groups; i.e., those groups $A$ such that there exists a faithful properly discontinuous action $A \times \mathbf{R}^{n} \rightarrow \mathbf{R}^{n}$ by isometries of Euclidean space $\mathbf{R}^{n}$, where $n$ comes from 4.1(b). So by Proposition 2.3 any space having $A$ for a fundamental group satisfies the Fibered Conjecture 1.7 for the functors $\mathscr{P}_{*}()$ and $\mathscr{P}^{\text {diff }}()$. Let $\tau: \Gamma \rightarrow A$ denote the epimorphism of $(4.2)$ with kernel $\Gamma^{\prime}$. Note also that it follows from 4.1(a), and from the induction hypothesis for our present proof, that any space $Z$ with $\pi_{1} Z$ isomorphic to $\tau^{-1}(H)$ for some $H \in \mathscr{G}(K(A, 1))$ also satisfies the Fibered Conjecture 1.7 for the functors $\mathscr{P}_{*}()$ and $\mathscr{P}_{*}^{\text {diff }}()$. Now replacing (4.2) by a fibration of Eilenberg-McLane spaces

$$
K\left(\Gamma^{\prime}, 1\right) \rightarrow K\left(\pi_{1} X, 1\right) \rightarrow K(A, 1),
$$

and applying Proposition 2.2 to (4.3), we conclude that $K\left(\pi_{1} X, 1\right)$ also satisfies the Fibered Conjecture 1.7 for the functors $\mathscr{P}_{*}()$ and $\mathscr{P}_{*}^{\text {diff }}()$. Now the induction step in our present argument is completed by applying Corollary 2.2.1.

This completes the proof of Proposition 2.4 for spaces which have finitely generated virtually nilpotent fundamental groups. 
The proof of Proposition 2.4 for general virtually poly- $Z$ groups depends on the following lemma, which will be proven at the end of this section. (See [17, Lemma 2.1] for a result related to this lemma.)

4.4. Lemma. Suppose that $\Gamma$ is a finitely generated virtually poly- $\mathbf{Z}$ group with $\operatorname{vcd}(\Gamma)=k<\infty$. Then there is a short exact sequence of groups

$$
1 \rightarrow \Gamma^{\prime} \rightarrow \Gamma \rightarrow A \rightarrow 1,
$$

where $\Gamma^{\prime}$, A satisfy the following properties.

(a) $\Gamma^{\prime}$ is either a finite group or is a virtually poly-Z group with $\operatorname{vcd}\left(\Gamma^{\prime}\right) \leq$ $k-2$.

(b) Either $A$ is as in 4.1(b), or $A$ satisfies (c) and (d).

(c) There is a short exact sequence

$$
1 \rightarrow A^{\prime} \rightarrow A \stackrel{\alpha}{\rightarrow} B \rightarrow 1 .
$$

where $A^{\prime}$ is a crystallographic group (see 4.1(b)) and $B$ is either the infinite cyclic group $\mathbf{Z}$ or the infinite dihedral group $D_{\infty}$.

(d) If $B=D_{\infty}$ in (c), then for any elements $a_{1} \in A^{\prime}, a_{2} \in A$, such that both $a_{1}$ and $\alpha\left(a_{1}\right)$ have infinite order, we must have that $a_{2} a_{1} a_{2}^{-1} \neq a_{1}$ and $a_{2} a_{1} a_{2}^{-1} \neq a_{1}^{-1}$. Moreover, there is no proper subgroup $G \subset A^{\prime}$ such that $G$ is both a normal subgroup of $A$ and $\operatorname{vcd}(G)<\operatorname{vcd}\left(A^{\prime}\right)$.

Proof of Proposition 2.4. The proof proceeds by induction over the value $\operatorname{vcd}\left(\pi_{1} X\right)$ as before. We suppose that the Fibered Conjecture 1.7 is true for any space $Y$ such that $\pi_{1} Y$ is virtually poly-Z and $\operatorname{vcd}\left(\pi_{1} Y\right) \leq r$ and for the functors $\mathscr{P}_{*}(), \mathscr{P}_{*}^{\text {diff }}()$. Let $X$ denote a space for which $\pi_{1} X$ is a virtually poly-Z group with $\operatorname{vcd}\left(\pi_{1} X\right)=r+1$. If $\pi_{1} X$ satisfies $4.1(a)$, (b) then the induction step is carried through as for the virtually nilpotent case.

If $\pi_{1} X$ satisfies $4.2(\mathrm{a}),(\mathrm{c})$, (d) then there is considerably more work to do. We note that by $4.2(\mathrm{a}),(\mathrm{c}),(\mathrm{d})$ there are short exact sequences

$$
\begin{aligned}
& \text { (a) } 1 \rightarrow \Gamma^{\prime} \rightarrow \pi_{1} X \rightarrow A \rightarrow 1, \\
& \text { (b) } 1 \rightarrow A^{\prime} \rightarrow A \stackrel{\alpha}{\rightarrow} B \rightarrow 1,
\end{aligned}
$$

where $A^{\prime}, A, B, \Gamma^{\prime}$ are as in $4.4(\mathrm{a}),(\mathrm{c}),(\mathrm{d})$.

4.6. Claim. There is a group action $A \times \mathbf{R}^{n+1} \rightarrow \mathbf{R}^{n+1}$, which satisfies the following properties.

(a) $A \times \mathbf{R}^{n+1} \rightarrow \mathbf{R}^{n+1}$ is a properly discontinuous co-compact action by affine motions of Euclidean space which preserve the product structure $\mathbf{R}^{n+1}=\mathbf{R}^{n} \times$ $\mathbf{R}$. Thus $A \times \mathbf{R}^{n+1} \rightarrow \mathbf{R}^{n+1}$ is the diagonal action for two separate actions $\psi_{1}: A \times \mathbf{R}^{n} \rightarrow \mathbf{R}^{n}$ and $\psi_{2}: A \times \mathbf{R} \rightarrow \mathbf{R}$.

(b) The restricted action $\psi_{1}: A^{\prime} \times \mathbf{R}^{n} \rightarrow \mathbf{R}^{n}$ is a properly discontinuous cocompact action by isometries of Euclidean space.

(c) $\psi_{2}$ acts through the quotient group $B$ of $A$, and $\psi_{2}: B \times \mathbf{R} \rightarrow \mathbf{R}$ is a properly discontinuous co-compact action by isometries of the real line.

To verify Claim 4.6 we note that there are only the following two cases to consider. 
Case 1. In this case we suppose that $B=\mathbf{Z}$. Choose $g \in A$ so that $\alpha(g)$ generates $B$. Use 4.4(c) and Zassenhaus's existence theorem [59] to get the action $\psi_{1}: A^{\prime} \times \mathbf{R}^{n} \rightarrow \mathbf{R}^{n}$. Since crystallographic actions of $A^{\prime}$ are unique up to affine equivalence by Bieberbach's isomorphism theorem (cf. [56]), choose an affine isomorphism $h: \mathbf{R}^{n} \rightarrow \mathbf{R}^{n}$ such that

$$
\psi_{1}\left(\operatorname{gag}^{-1}, h(x)\right)=h\left(\psi_{1}(a, x)\right)
$$

for all $a \in A^{\prime}$ and $x \in \mathbf{R}^{n}$. By setting

$$
\psi_{1}(g, x)=h(x), \quad \text { for all } x \in \mathbf{R}^{n}
$$

we extend the definition of $\psi_{1}$ to $\psi_{1}: A \times \mathbf{R}^{n} \rightarrow \mathbf{R}^{n}$. Define $\psi_{2}: B \times \mathbf{R} \rightarrow \mathbf{R}$ by

$$
\psi_{2}\left(\alpha\left(g^{k}\right), x\right)=x+k \text { for all } x \in \mathbf{R} \text { and } k \in \mathbf{Z} \text {. }
$$

Case 2. In this case we suppose that $B=D_{\infty}$. Recall that $\mathbf{Z}^{n}$ is a characteristic subgroup of the crystollographic group $A^{\prime}$ with finite quotient. Hence $Z^{n}$ is a normal subgroup of $A$. Consider the short exact sequence of groups

$$
1 \rightarrow \mathbf{Z}^{n} \rightarrow A \rightarrow \mathscr{G} \rightarrow 1,
$$

where $\mathscr{G}=A / Z^{n}$. Note $\alpha: A \rightarrow D_{\infty}$ factors through and epimorphism $\bar{\alpha}: \mathscr{G} \rightarrow D_{\infty}$, which has the finite group $A^{\prime} / \mathbf{Z}^{n}$ for kernel. Since $D_{\infty}$ is the free product of $\mathbf{Z}_{2}$-the cyclic group of order 2 -with itself, we obtain an amalgamated free product structure

$$
\mathscr{G}=\mathscr{A}_{0} * \mathscr{B} \mathscr{A}_{1}
$$

where $\mathscr{A}_{0}, \mathscr{A}_{1}$, and $\mathscr{B}$ are finite subgroups of $\mathscr{G}$. The extension 4.6 .1 determines a class $\Theta \in H^{2}\left(\mathscr{G} ; \mathbf{Z}^{n}\right)$, where $H^{2}\left(\mathscr{G} ; \mathbf{Z}^{n}\right)$ is a finite group because of the Mayer-Vietoris exact sequence

$$
H^{2}\left(\mathscr{A}_{0} ; \mathbf{Z}^{n}\right) \oplus H^{2}\left(\mathscr{A}_{1} ; \mathbf{Z}^{n}\right) \leftarrow H^{2}\left(\mathscr{G} ; \mathbf{Z}^{n}\right) \leftarrow H^{1}\left(\mathscr{B} ; \mathbf{Z}^{n}\right) .
$$

Consequently, $\Theta$ has finite order. The action of $\mathscr{G}$ on $\mathbf{Z}^{n}$ is a faithful representation into $\mathrm{GL}_{n}(\mathbf{Z})$ because of $4.4(\mathrm{~d})$. Hence the induced representation into $\mathrm{GL}_{n}(\mathbf{R})$ is also faithful. (We may assume that $\mathscr{G} \rightarrow \mathrm{GL}_{n}(\mathbf{R})$ sends the finite group $A^{\prime} / \mathbf{Z}^{n}$ into the orthogonal group $O_{n}(\mathbf{R})$ since the maximal compact subgroups of $\mathrm{GL}_{n}(R)$ are all conjugate.) This map of $\mathscr{G}$-modules $\mathbf{Z}^{n} \rightarrow \mathbf{R}^{n}$ induces a map in cohomology $H^{2}\left(\mathscr{G} ; \mathbf{Z}^{n}\right) \rightarrow H^{2}\left(\mathscr{G} ; \mathbf{R}^{n}\right)$, which sends $\Theta$ to a class $\Theta^{\prime} \in H^{2}\left(\mathscr{G} ; \mathbf{R}^{n}\right)$. Let

$$
1 \rightarrow \mathbf{R}^{n} \rightarrow \mathbf{A} \rightarrow \mathscr{G} \rightarrow 1
$$

be the group extension that $\Theta^{\prime}$ determines. There is also a group of homomorphism $f: A \rightarrow \mathbf{A}$ such that the following diagram commutes:

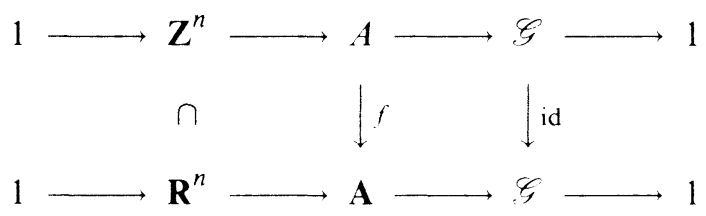


Here $\subset$ denotes the standard inclusion but perhaps composed with an element of $\mathrm{GL}_{n}(\mathbf{R})$. Consequently, $f$ is a monomorphism.

But $\Theta^{\prime}=0$ since it has finite order in the $\mathbf{R}$-module $H^{2}\left(\mathscr{G} ; \mathbf{R}^{n}\right)$. Therefore 4.6.2 splits; i.e., $\mathbf{A}$ is a semidirect product $\mathbf{R}^{n} \rtimes \mathscr{G}$ and consequently a subgroup of $\mathbf{R}^{n} \rtimes \mathrm{GL}_{n}(\mathbf{R})$-the group of all affine motions of $\mathbf{R}^{n}$. The composite of $f$ with this inclusion defines the action $\psi_{1}$. The action $\psi_{2}$ is defined by realizing $B=D_{\infty}$ as the crystallographic group generated by the two reflections of $\mathbf{R}$ in 0 and 1 respectively.

This completes the proof of Claim 4.6.

4.7. Definition. A group $A$ is called a special affine group if there is a group action $A \times \mathbf{R}^{n+1} \rightarrow \mathbf{R}^{n+1}$ which satisfies $4.6(\mathrm{a})-(\mathrm{c})$ (where $A, A^{\prime}, B$ of 4.6 are as in $4.4(\mathrm{c}))$.

By using 4.4(a), 4.6(a)-(c), Proposition 2.2, Corollary 2.2.1, and Theorem 4.8, we can fashion an induction argument, similar to the proof of Proposition 2.4 given above for the case of finitely generated virtually nilpotent groups, which will complete the proof Proposition 2.4. (Note that Theorem 4.8 now plays the same role that Proposition 2.3 did in the proof for finitely generated virtually nilpotent groups.)

This completes the proof of Proposition 2.4.

The proof of the following theorem will be given in the separate paper [23].

4.8. Theorem. Let $X$ denote a connected $C W$-complex such that $\pi_{1} X$ is a special affine group. Then $X$ satisfies the Fibered Conjecture 1.7 for the functors $\mathscr{P}_{*}\left(\right.$ ) and $\mathscr{P}_{*}^{\text {diff }}($ ) .

In the remainder of this section we give the proofs for Lemmas 4.1 and 4.4.

Proof of Lemma 4.1. We will use the following two well-known facts about the class $\mathscr{C}$ of finitely generated torsion-free nilpotent groups.

Fact 1. The class $\mathscr{C}$ is closed with respect to subgroups.

Fact 2. If $G \in \mathscr{C}$ and $G$ is not abelian, then $\operatorname{vcd}(G /[G, G])>1$.

(Note that both these facts are false for the larger class $\mathscr{C}^{\prime}$ of all finitely generated torsion-free solvable groups.) Fact 1 can be deduced from statements $2.18,3.7$ and 4.2 in [42]. Fact 2 follows from 2.18 of [42] together with the corresponding fact for simply connected nilpotent Lie groups. (Hint: All 2dimensional connected nilpotent Lie groups are abelian.)

Let $G \subset \Gamma$ denote a normal torsion-free subgroup of finite index which is finitely generated and nilpotent. Note that it follows from Fact 1 that any subgroup of $G$ is a torsion-free finitely generated nilpotent group. We complete the proof of 4.1 in the following two steps.

Step 1. In this step we suppose that $G$ is an abelian group, that is, $\Gamma$ is finitely generated and virtually abelian. Any such group contains a unique maximal normal finite subgroup $\Gamma^{\prime}$, and $\Gamma / \Gamma^{\prime}$ is crystallographic (i.e., satisfies $4.1(\mathrm{~b})$ ). Hence the short exact sequence

$$
1 \rightarrow \Gamma^{\prime} \rightarrow \Gamma \rightarrow \Gamma / \Gamma^{\prime} \rightarrow 1
$$

is seen to satisfy $4.1(a),(b)$. 
Step 2. We may assume in this case that $[G, G] \neq 1$. Since $[G, G]$ is a characteristic subgroup of $G$, it is normal in $\Gamma$. Using Fact 2 , we see that $\Gamma /[G, G]$ is finitely generated, virtually abelian with $\operatorname{vcd}(\Gamma /[G, G])>1$. By step $1, \Gamma /[G, G]$ has a crystallographic quotient $\Gamma / \Gamma^{\prime}$ with $\Gamma^{\prime} /[G, G]$ finite. Hence the short exact sequence

$$
1 \rightarrow \Gamma^{\prime} \rightarrow \Gamma \rightarrow \Gamma / \Gamma^{\prime} \rightarrow 1
$$

is again seen to satisfy $4.1(\mathrm{a}),(\mathrm{b})$.

This completes the proof of Lemma 4.1.

Proof of Lemma 4.4. Let $G \subset \Gamma$ denote a normal subgroup of finite index which is a poly- $Z$ group. It follows that any subgroup of $G$ is also a poly- $Z$ (cf. $[42, \S 4]$ ). We assume (by 4.1) that $[G, G] \neq 1$. Set $G^{\prime}=[G, G]$. Note that $1 \leq \operatorname{vcd}\left(G / G^{\prime}\right)<\infty$. Since $G^{\prime}$ is a characteristic subgroup of $G$, it is normal in $\Gamma$ and $\Gamma / G^{\prime}$ is a finitely generated and virtually abelian. Let $\pi / G^{\prime}$ denote the unique maximal finite normal subgroup in $\Gamma / G^{\prime}$. Then $\Gamma / \pi$ is a crystallographic group with $d=\operatorname{vcd}(\Gamma / \pi) \geq 1$. If $d \geq 2$, then we set $\Gamma^{\prime}=\pi$ and $A=\Gamma / \pi$ in 4.4 and we are done.

If $d=1$, then more work is required. In this case, note that $\Gamma / \pi$ is either $\mathbf{Z}$ or $D_{\infty}$. Let $G^{\prime \prime}=\left[G^{\prime}, G^{\prime}\right]$. Then $G^{\prime \prime}$ is normal in $\Gamma$ since it is characteristic in $G$. Note that $1 \leq \operatorname{vcd}\left(G^{\prime} / G^{\prime \prime}\right)<\infty$ since $G^{\prime}$ is a nontrivial poly-Z group. Hence $\pi / G^{\prime \prime}$ is both finitely generated and virtually abelian. Let $\pi_{1} / G^{\prime \prime}$ be the unique maximal finite normal subgroup in $\pi / G^{\prime \prime}$. Since $\pi_{1} / G^{\prime \prime}$ is characteristic in $\pi / G^{\prime \prime}$, it is normal in $\Gamma / G^{\prime \prime}$. Hence $\pi_{1}$ is normal in $\Gamma$. Therefore $\pi / \pi_{1}$ is a nontrivial crystallographic normal subgroup of $\Gamma / \pi_{1}$ whose quotient is the crystallographic group $\Gamma / \pi$. In the case that $\Gamma / \pi=\mathbf{Z}$, set $\Gamma^{\prime}=\pi_{1}, A=\Gamma / \pi_{1}$ and $A^{\prime}=\pi / \pi_{1}$ in 4.4 , and we are done.

We may therefore assume while completing the proof of 4.4 that $\Gamma / \pi=D_{\infty}$ and that $\Gamma$ has no virtually abelian quotient whose vcd $>1$. We proceed to construct a finite increasing sequence $\pi_{i}, i=1,2, \ldots, n$, of normal subgroups of $\Gamma$ starting with the already constructed $\pi_{1}$ and ending with the desired group $\Gamma^{\prime}$ which will be a proper subgroup of $\pi$, i.e.,

$$
\pi_{1} \subset \pi_{2} \subset \cdots \subset \pi_{n}=\Gamma^{\prime} \varsubsetneqq \pi .
$$

After this construction, we will complete the proof of 4.4 by setting $\Gamma^{\prime}=\pi_{n}$, $A=\Gamma / \pi_{n}$, and $A^{\prime}=\pi / \pi_{n}$. The construction of $\pi_{i+1}$ from $\pi_{i}$ is done in two steps. The first step constructs an intermediate

$$
\pi_{i} \subset \pi^{i} \subset \pi_{i+1}
$$

with $\pi^{i}$ also normal in $\Gamma$. Each quotient $\pi / \pi_{i}$ or $\pi / \pi^{i-1}$ will be crystallographic. We now do the inductive step by constructing $\pi^{i}$ and $\pi_{i+1}$.

If $\pi / \pi_{i}$ contains a nontrivial $\infty$-index subgroup $S_{i} / \pi_{i}$ which is normal in $\Gamma / \pi_{i}$, let $\pi^{i} / S_{i}$ be the unique maximal finite normal subgroup in $\pi / S_{i}$. If no such group $S_{i}$ exists, set $\pi^{i}=\pi_{i}$. 
To construct $\pi_{i+1}$, let $T_{i}$ denote the subgroup of pure translations in the crystallographic group $\pi / \pi^{i}$. Recall that $T_{i}$ is a finitely generated, free abelian, characteristic subgroup of finite index in $\pi / \pi^{i}$. Let $\mathscr{S}_{i}$ be the subset of $T_{i}$ consisting of all elements $a \in T_{i}$ such that there exists $b \in \Gamma / \pi^{i}$ with

(i) $b a b^{-1}=a$, and

(ii) the image of $b$ in $D_{\infty}=\Gamma / \pi$ has infinite order.

It can be shown that $\mathscr{S}_{i}$ is a normal subgroup of $\Gamma / \pi^{i}$. Hence $\mathscr{S}_{i}=S^{i} / \pi^{i}$, where $S^{i}$ is a normal subgroup of $\Gamma$ with $\pi^{i} \subset S^{i} \subset \pi$. Let $\pi_{i+1} / S^{i}$ be the unique maximal normal finite subgroup in $\pi / S^{i}$. It can also be shown that $\mathscr{S}_{i}$ has infinite index in $T^{i}$ since otherwise $\Gamma / \pi^{i}$ would be a virtually abelian quotient of $\Gamma$ with $\operatorname{vcd}\left(\Gamma / \pi^{i}\right)>1$. Therefore, $\pi_{i+1}$ is a proper subgroup of $\pi$ and we have completed the inductive step in the construction.

Note that if $\operatorname{vcd}\left(\pi_{i+1} / \pi_{i}\right)=0$, then $\pi_{i}=\pi_{i+1}$. The construction consequently must stop after a finite number of steps $n$. This completes the proof of Lemma 4.4 .

\section{APPENDIX}

In this appendix we give an axiomatic treatment for the concept of "universal $(\Gamma, \mathscr{C})$-space", where $\Gamma$ denotes a (discrete) group and $\mathscr{C}$ denotes a collection of subgroups of $\Gamma$. We also prove Proposition 2.2 (see Remark A.12) and verify several claims made in 1.6.1, 1.6.2, and 1.6.4 (see Remark A.11). In addition, we prove the following useful result (see A.8): If Conjecture 1.7 is true for the connected CW-complex $X$ then it is also true for any connected covering space of $X$. We begin with some notation and terminology.

A.1. Notation and terminology. Let $\Gamma$ be a (discrete) group, and let $X$ be a (left) $\Gamma$-space. If $S \subset \Gamma$ is a subgroup, then $X^{S}=\{x \in X: s x=x$ for all $s \in S\}$, i.e., $X^{S}$ is the set of fixed points of $S$. If $x \in X$, then $\Gamma^{x}$ is the isotropy subgroup fixing $x$, i.e., $\Gamma^{x}=\{g \in \Gamma: g x=x\}$. More generally, let $A \subset X$ be a subspace. Then $\Gamma^{A}$ is the isotropy subgroup (pointwise) fixing $A$, i.e., $\Gamma^{A}=\bigcap_{a \in A} \Gamma^{a}$. A sometimes larger subgroup is $\Gamma^{(A)}=\{g \in \Gamma: g A=A\}$, i.e., $\Gamma^{(A)}$ is the subgroup leaving $A$ invariant. Let $\mathscr{C}$ be a (nonempty) set of subgroups of $\Gamma$. Then $X$ is said to be $\mathscr{C}$-contractible if and only if $X^{S}$ is nonempty and contractible for all $S \in \mathscr{C}$. Furthermore, $X$ is $\mathscr{C}$-free if and only if $\Gamma^{x} \in \mathscr{C}$ for each $x \in X$. A cellular action of $\Gamma$ on $X$ is one such that $X$ is a regular cell complex and the following two properties hold. (See [10, p. 3] for the precise definition of a regular cell complex.) First, for each $g \in \Gamma$, the homeomorphism $x \rightarrow g x$ maps cells onto cells. Second, for each (closed) cell $\sigma$ of $X, \Gamma^{(\sigma)}=\Gamma^{\sigma}$. In the case of cellular actions, note that $\Gamma^{\sigma}=\Gamma^{x}$ for each $x \in \operatorname{Int} \sigma$. Consequently, $\left\{\Gamma^{\sigma}: \sigma\right.$ is a cell of $\left.X\right\}=\left\{\Gamma^{x}: x \in X\right\}$. A cellular $\Gamma$-complex which is both $\mathscr{C}$-free and $\mathscr{C}$-contractible is called a universal $(\Gamma, \mathscr{C})$-space or a $\mathscr{E}(\Gamma, \mathscr{C})$-space. A nonempty class of subgroups $\mathscr{C}$ of $\Gamma$ is full if and only if it is closed with respect to both subgroups and conjugates. 
The following two theorems will be proven in this appendix.

A.2. Theorem. Let $X$ and $Y$ be $\Gamma$-spaces such that $X$ is a cellular $\mathscr{C}$-free space and $Y$ is $\mathscr{C}$-contractible. Then there is a $\Gamma$-map from $X$ to $Y$. Furthermore any two $\Gamma$-maps are homotopic through $\Gamma$-maps.

A.2.1. Remark. Connolly and Koz'niewski earlier proved A.2 in [9] for the important class $\mathscr{C}=\mathscr{G}^{\prime}(B \Gamma)$ of all finite subgroups of $\Gamma$.

A.2.2. Remark. The maps and homotopies posited in A.2 can be constructed to be cellular when the action of $\Gamma$ on $Y$ is cellular.

A.3. Theorem. Let $\mathscr{C}$ be a full class of subgroups of $\Gamma$. Then there exists a $\mathscr{E}(\Gamma, \mathscr{C})$-space.

Proof of Theorem A.2. It suffices to prove the following relative extension result. Let $K$ be a $\Gamma$-invariant subcomplex of $X$ and $f: K \rightarrow Y$ be a $\Gamma$-map. Then there exists a $\Gamma$-map $\hat{f}: X \rightarrow Y$ whose restriction to $K$ is $f$.

Our proof of this relative statement is a standard obstruction theory argument. We proceed to (on $n$ ) construct inductively $\Gamma$-equivariant extensions $f_{n}: K \cup X_{n} \rightarrow Y$, where $X_{n}$ denotes the $n$-skeleton of $X$. The constructions are such that $f_{n+1}$ restricted to $K \cup X_{n}$ is $f_{n}$ (and $f_{-1}=f$ ). We start by constructing $f_{0}$. Pick a collection of vertices $S_{0}$ of $X_{0}-K$ such that each $\Gamma$ orbit of any vertex in $X_{0}-K$ is represented in $S_{0}$ exactly once. For each $v \in S_{0}$, pick a point $\hat{v} \in Y^{T}$, where $T=\Gamma^{v}$. Note that $Y^{T}$ is nonempty since $\Gamma^{v} \in \mathscr{C}$. Now define $f_{0}(g v)=g \hat{v}$, for each $v \in S_{0}$ and $g \in \Gamma$. This starts our inductive construction. Now we assume that $f_{n}$ has been constructed, and we proceed to construct $f_{n+1}$. Let $S_{n+1}$ be a set of $n+1$ cells $\sigma$ with $\operatorname{Int}(\sigma)$ in $X_{n+1}-K$ such that each $\Gamma$-orbit of an $n+1$ cell $\tau$ with $\operatorname{Int}(\tau)$ in $X_{n+1}-K$ is represented exactly once in $S_{n+1}$. For each $n+1$ cell $\sigma \in S_{n+1}$, let $\psi_{\sigma}: \partial \sigma \rightarrow Y$ denote the restriction of $f_{n}$ to $\partial \sigma$. Note that image $\left(\psi_{\sigma}\right) \subset Y^{T}$, where $T=\Gamma^{\sigma}$ since

$$
\Gamma^{f_{n}(x)} \supset \Gamma^{x} \supset \Gamma^{\sigma}, \quad \text { for all } x \in \partial \sigma
$$

and hence

$$
f_{n}(x) \in Y^{R} \subset Y^{T}, \quad \text { where } R=\Gamma^{f_{n}(x)} .
$$

Note that $Y^{T}$ is contractible since $\Gamma^{\sigma} \in \mathscr{C}$. Hence we can pick an extension $\hat{\psi}_{\sigma}: \sigma \rightarrow Y^{T}$ of $\psi_{\sigma}$. We now define $f_{n+1}$ by the following formulae:

$$
f_{n+1}(g x)=g \hat{\psi}_{\sigma}(x), \quad \text { if } x \in \sigma \text { where } \sigma \in S_{n+1}
$$

and

$$
f_{n+1}(x)=f_{n}(x), \quad \text { if } x \in K \cup X_{n} .
$$

This completes the proof of Theorem A.2.

Proof of Theorem A.3. We will construct a $\mathscr{E}(\Gamma, \mathscr{C})$-space, which we will denote by $X$. The space $X$ is the geometric realization of a semisimplicial complex also denoted by $X$. An $n$-simplex in $X$ is by definition an array

$$
\sigma=g_{0} S_{0}\left(g_{1} S_{1}, g_{2} S_{2}, \ldots, g_{n} S_{n}\right),
$$


where each $S_{i} \in \mathscr{C}$ and $g_{i} S_{i}$ is a left coset of $S_{i}$ in $\Gamma$. These cosets must satisfy the following conditions:

$$
S_{i-1} g_{i} \subset g_{i} S_{i}, \quad \text { for } i=1,2, \ldots, n .
$$

In particular, the vertices are arrays $g S()$, where $S \in \mathscr{C}$. The group $\Gamma$ acts on the set of $n$-simplices of $X$ by the formula

$$
g \sigma=g\left(g_{0} S_{0}\left(g_{1} S_{1}, \ldots, g_{n} S_{n}\right)\right)=\left(g g_{0}\right) S_{0}\left(g_{1} S_{1}, \ldots, g_{n} S_{n}\right) .
$$

The face operators $\partial_{i}$ are defined as

$$
\begin{aligned}
\partial_{0} \sigma & =g_{0} g_{1} S_{1}\left(g_{2} S_{2}, \ldots, g_{n} S_{n}\right), \\
\partial_{n} \sigma & =g_{0} S_{0}\left(g_{1} S_{1}, \ldots, g_{n-1} S_{n-1}\right), \\
\partial_{i} \sigma & =g_{0} S_{0}\left(g_{1} S_{1}, \ldots, g_{i-1} S_{i-1}, g_{i} g_{i+1} S_{i+1}, g_{i+2} S_{i+2}, \ldots, g_{n} S_{n}\right)
\end{aligned}
$$

when $1 \leq i<n$. Note that (2) implies that

$$
S_{i-1} g_{i} g_{i+1} \subset g_{i} g_{i+1} S_{i+1}, \quad \text { for } i=1,2, \ldots, n-1,
$$

which shows that (4) is well defined. Also note that each face operator is a $\Gamma$-equivariant map.

Note that $X$ is clearly a cellular $\Gamma$-space and that for each simplex $\sigma$ as in (1), we have

$$
\Gamma^{\sigma}=g_{0} S_{0} g_{0}^{-1}
$$

Since $\mathscr{C}$ is closed under conjugation, we see from (6) that $X$ is $\mathscr{C}$-free. Also note that, for each $S \in \mathscr{C}, X^{S}$ is the subcomplex of $X$ consisting of all simplices $\sigma$ such that $S \subset \Gamma^{\sigma}$. Combining this fact with (1) and (6), we see that $X^{S}$ consists of all simplices $\sigma$ (where $\sigma=g_{0} S_{0}\left(g_{1} S_{1}, \ldots, g_{n} S_{n}\right)$ ) such that

$$
S g_{0} \subset g_{0} S_{0}
$$

We simplicially define a contracting homotopy $s$ of $X^{S}$ to the vertex $S($ ) via the following formula in which $\sigma=g_{0} S_{0}\left(g_{1} S_{1}, \ldots, g_{n} S_{n}\right)$ is an arbitrary $n$-simplex in $X^{S}$ :

$$
s \sigma=S\left(g_{0} S_{0}, g_{1} S_{1}, \ldots, g_{n} S_{n}\right)
$$

Note that $\partial_{0} s=$ id and $\partial_{i+1} s=s \partial_{i}$ for $i>0$. This shows that $X^{S}$ is contractible and, hence, $X$ is $\mathscr{C}$-contractible.

This completes the proof of Theorem A.3.

A.4. Remark. The above construction of an $\mathscr{E}(\Gamma, \mathscr{E})$ is the bar resolution when $\mathscr{C}=\{1\}$.

The remainder of this appendix is devoted to verifying some of the claims made in 1.6.1, 1.6.2, and 1.6.4 above and to the proof of Proposition 2.2.

We begin with the following useful result, which is an immediate consequence of the definitions. 
A.5. Proposition. Let $\Gamma$ be a (discrete) group, and let $\mathscr{C}, \mathscr{C}^{\prime}$ be two full classes of subgroups of $\Gamma$ with $\mathscr{C}^{\prime} \subset \mathscr{C}$. Let $\pi \subset \Gamma$ be a subgroup, and set $\mathscr{C}_{\pi}=\{S \cap \pi: S \in \mathscr{C}\}$. Then $\mathscr{C}_{\pi}$ is a full class of subgroups of $\pi$ with $\mathscr{C}_{\pi} \subset \mathscr{C}$. Let $\mathscr{E}$ be a cellular $\Gamma$-space. Then by restriction it is also a cellular $\pi$-space. Furthermore, if $\mathscr{E}$ is $\mathscr{C}$-free, then it is also $\mathscr{C}_{\pi}$-free; and if $\mathscr{E}$ is $\mathscr{C}$-contractible, then it is also $\mathscr{C}_{\pi}$-contractible. Consequently, universal $(\Gamma, \mathscr{C})$-spaces are also universal $\left(\pi, \mathscr{C}_{\pi}\right)$-spaces. Let $\mathscr{E}$ and $\mathscr{E}^{\prime}$ be universal $(\Gamma, \mathscr{C})$ and $\left(\Gamma, \mathscr{C}^{\prime}\right)$ spaces respectively. Then $\mathscr{E}^{\prime} \times \mathscr{E}$, equipped with the diagonal $\Gamma$-action, is a universal $\left(\Gamma, \mathscr{C}^{\prime}\right)$-space.

A.5.1. Remark. The cartesian product of two regular cell complexes is always given the weak topology with respect to compact subsets. This makes it also a regular cell complex. There are two important cases when this topology agrees with the ordinary product topology. The first case is when at least one of the two factors is locally compact. The second is when both factors have only countably many cells. (This second case is pertinent to the construction of the fiber bundles in the proof of Theorem 2.1.) See [10, Chapter 4] for a detailed discussion.

Let $\mathscr{S}_{*}(\mathrm{)}$ be one of the $\Omega$-spectra-valued functors of 1.1-1.3. If $p: Y \rightarrow X$ is a simplicially stratified fibration, consider the commutative diagram

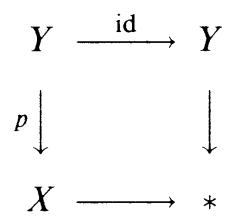

It induces a map of spectra

$$
A: \mathbb{H}_{*}\left(X, \mathscr{S}_{*}(p)\right) \rightarrow \mathscr{S}_{*}(Y)
$$

which we call its assembly map. The following fact is easily verified using that $\pi_{n}\left(X_{1} V X_{2}\right) \cong \pi_{n}\left(X_{1}\right) \oplus \pi_{n}\left(X_{2}\right)$ for spectra $X_{i}$.

A.6. Lemma. When $X$ is a discrete space, the assembly map $A: \mathbb{H}_{*}\left(X, \mathscr{S}_{*}(p)\right)$ $\rightarrow \mathscr{S}_{*}(Y)$ is an equivalence of $\Omega$-spectra.

Let $q_{E}: E \times D^{n} \rightarrow D^{n}$ denote projection onto the second factor (the $n$ dimensional ball). Then $\mathbb{H}_{*}\left(D^{n}, S^{n-1} ; \mathscr{S}_{*}\left(q_{E}\right)\right)$ is equivalent to the $\Omega$-spectrum $\mathscr{S}_{*+n}(E)$. Using this fact together with the axiomatic characterization of $\mathbb{H}_{*}\left(Y ; \mathscr{S}_{*}(p)\right)$ and Lemma A.6, the following extension of Lemma A.6 can be proven.

A.6.1. Addendum to A.6. Let $p: E \rightarrow X$ be a continuous map onto the discrete space $X$, and let $p \times$ id denote the induced map $E \times D^{n} \rightarrow X \times D^{n}$. Then

$$
\mathbb{H}_{*}\left(q_{X}\right): \mathbb{H}_{*}\left(X \times D^{n}, X \times S^{n-1} ; \mathscr{S}_{*}(p \times \mathrm{id})\right) \rightarrow \mathbb{H}_{*}\left(D^{n}, S^{n-1} ; \mathscr{S}_{*}\left(q_{E}\right)\right)
$$

is an equivalence of $\Omega$-spectra. 
Let $p: Y \rightarrow X$ and $p^{\prime}: Y^{\prime} \rightarrow X^{\prime}$ be simplicially stratified fibrations and $\varphi \in \operatorname{Mor}\left(p^{\prime}, p\right)$; i.e., $\varphi$ is a commutative diagram

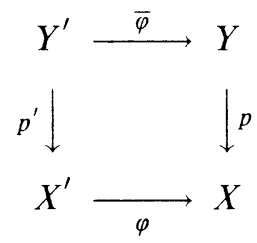

of continuous maps. In addition, assume that $\varphi: X^{\prime} \rightarrow X$ is a simplicial map. The following is also easily verified in whose statement $X_{n}, X_{n}^{\prime}$ denote the $n$-skeletons of $X, X^{\prime}$ respectively. (When $X$ is infinite dimensional, a limit argument is needed.)

A.7. Lemma. If for each integer $n$ the map

$$
\mathbb{H}_{*}(\varphi): \mathbb{H}_{*}\left(X_{n}^{\prime}, X_{n-1}^{\prime} ; \mathscr{S}_{*}\left(p^{\prime}\right)\right) \rightarrow \mathbb{H}_{*}\left(X_{n}, X_{n-1} ; \mathscr{S}_{*}(p)\right)
$$

is an equivalence of $\Omega$-spectra, then the map

$$
\mathbb{H}_{*}(\varphi): \mathbb{H}_{*}\left(X^{\prime} ; \mathscr{S}_{*}\left(p^{\prime}\right)\right) \rightarrow \mathbb{H}_{*}\left(X ; \mathscr{S}_{*}(p)\right)
$$

is also an equivalence of $\Omega$-spectra.

A.7.1. Remark. The conclusion of Lemma A.7 remains true when $X_{n}, X_{n}^{\prime}$ are any filtrations of $X, X^{\prime}$ by subcomplexes satisfying the following:

(i) $\varphi\left(X_{n}^{\prime}\right) \subset X_{n}$;

(ii) $\bigcup_{n} X_{n}=X$ and $\bigcup_{n} X_{n}^{\prime}=X^{\prime}$.

A.8. Theorem. Let $\Gamma$ be a (discrete) group and $\pi$ be a subgroup. If the Fibered Isomorphism Conjecture 1.7 holds for all Serre fibrations over $B \Gamma$, then it also holds for all Serre fibrations over $B \pi$.

Proof of Theorem A.8. Let $\mathscr{C}$ be the class $\mathscr{G}(B \Gamma)$. Then $\mathscr{C}_{\pi}$ is $\mathscr{G}(B \pi)$. Let $\mathscr{E}$ be a universal $(\Gamma, \mathscr{C})$-space, and let $E$ be a universal $\Gamma$-space (i.e., universal for the class $\{1\})$. Then, by Proposition A.5, $\mathscr{E}$ and $E$ are also universal $\left(\pi, \mathscr{C}_{\pi}\right)$ and universal $\pi$-spaces respectively. Let $\xi$ be a fibration $\psi: Y \rightarrow B \pi$ where we identify $B \pi$ and $B \Gamma$ to $E / \pi$ and $E / \Gamma$ respectively. Let $\widetilde{Y} \rightarrow Y$ be the pullback of the universal cover $E \rightarrow B \pi$ along $\psi$. Then the pullback of $E \rightarrow B \Gamma$ along the composite $Y \rightarrow B \pi \rightarrow B \Gamma$ is the associated bundle $\Gamma \times{ }_{\pi} \tilde{Y} \rightarrow Y$.

Consider the simplicially stratified fibration

$$
p: \mathscr{E} \times_{\Gamma}\left(\Gamma \times_{\pi} \tilde{Y}\right) \rightarrow \mathscr{E} / \Gamma
$$

Since Conjecture 1.7 is true for all Serre fibrations over $B \Gamma$, the assembly map $A$ for $p$ is an equivalence of $\Omega$-spectra. But $p$ is the composite of the simplicially stratified fibration

$$
p^{\prime}: \mathscr{E} \times_{\Gamma}\left(\Gamma \times_{\pi} \tilde{Y}\right)=\mathscr{E} \times_{\pi} \tilde{Y} \rightarrow \mathscr{E} / \pi
$$


with the canonical quotient map $q: \mathscr{E} / \pi \rightarrow \mathscr{E} / \Gamma$. Hence the assembly map $A^{\prime}$ for $p^{\prime}$ is the composite of $A$ and

$$
\mathbb{H}_{*}(q): \mathbb{H}_{*}\left(\mathscr{E} / \pi ; \mathscr{S}_{*}\left(p^{\prime}\right)\right) \rightarrow \mathbb{H}_{*}\left(\mathscr{E} / \Gamma ; \mathscr{S}_{*}(p)\right)
$$

Note that $q \in \operatorname{Mor}\left(p^{\prime}, p\right)$ is determined by the commutative diagram

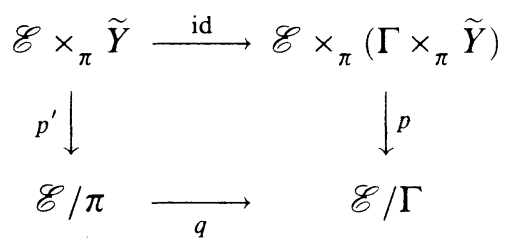

Hence it suffices to show that $\mathbb{H}_{*}(q)$ is an equivalence of $\Omega$-spectra. Let $\left\{X_{m}\right\}$ be a filtration of $\mathscr{E} / \Gamma$ by subcomplexes such that $X_{m}-X_{m-1}$ is a single open cell $\sigma_{m}$ and such that $\bigcup_{m} X_{m}=\mathscr{E} / \Gamma$. Such filtrations obviously exist when $\mathscr{E}$ is a countable complex. See Remark A.8.1 for the uncountable case. Let $X_{m}^{\prime}=q^{-1}\left(X_{m}\right)$. Then $X_{m}, X_{m}^{\prime}$ satisfy the conditions of Remark A.7.1 for $q: \mathscr{E} / \pi \rightarrow \mathscr{E} / \Gamma$. So we need only show that

$$
\mathbb{H}_{*}(q): \mathbb{H}_{*}\left(X_{m}^{\prime}, X_{m-1}^{\prime} ; \mathscr{S}_{*}\left(p^{\prime}\right)\right) \rightarrow \mathbb{H}_{*}\left(X_{m}, X_{m-1} ; \mathscr{S}_{*}(p)\right)
$$

is an equivalence of $\Omega$-spectra for each integer $m$. (Call this Condition (m).) First consider the cases where $\sigma_{m}$ is a 0 -cell. Using excision, we can identify $\mathbb{H}_{*}(q)$, in these cases, with the assembly map

$$
A: \mathbb{H}_{*}\left(q^{-1}\left(\sigma_{m}\right) ; \mathscr{S}_{*}\left(p^{\prime}\right)\right) \rightarrow \mathscr{S}_{*}\left(p^{-1}\left(\sigma_{m}\right)\right)=\mathbb{H}_{*}\left(\sigma_{m} ; \mathscr{S}_{*}(p)\right)
$$

Since $q^{-1}\left(\sigma_{m}\right)$ is discrete, Lemma A.6 shows that Condition $(\mathrm{m})$ holds when $\sigma_{m}$ is a 0 -cell. If $\sigma_{m}$ is an $m$-cell with $m>0$, then this argument can be modified by using Addendum A.6.1 in place of Lemma A.6 to verify Condition $(\mathrm{m})$. The details of this are left to the reader. This completes the proof of Theorem A.8.

A.8.1 Remark. When the filtration $\left\{X_{m}\right\}$ of $\mathscr{E} / \Gamma$ does not exist, a modified version of the above proof still works. The modification involves using the fact that $\mathbb{H}_{*}(\mathscr{E} / \Gamma ; \mathscr{S}(p))$ is the direct limit of $\mathbb{H}_{*}(K ; \mathscr{S}(p))$ as $K$ varies over the finite subcomplexes of $\mathscr{E} / \Gamma$.

Let $\mathscr{C}$ and $\mathscr{C}^{\prime}$ be two full classes of subgroups of a (discrete) group $\Gamma$ with $\mathscr{C}^{\prime} \subset \mathscr{C}$. We can define two absolute and one relative assembly maps $A_{f}$, $A_{\mathscr{C}}$, and $A_{\mathscr{C}^{\prime}, \mathscr{C}}$ as follows. Let $\mathscr{E}$ and $\mathscr{E}^{\prime}$ be universal $(\Gamma, \mathscr{C})$ and $\left(\Gamma, \mathscr{C}^{\prime}\right)$ spaces respectively, and let $E$ be a universal $\Gamma$-space. Consider the following three simplicially stratified fibrations:

$$
\begin{gathered}
p: \mathscr{E} \times_{\Gamma} E \rightarrow \mathscr{E} / \Gamma, \\
p^{\prime}: \mathscr{E}^{\prime} \times_{\Gamma} E \rightarrow \mathscr{E}^{\prime} / \Gamma, \\
*: E / \Gamma \quad \rightarrow * .
\end{gathered}
$$


We proceed to construct

$$
\begin{aligned}
A_{\mathscr{C}} & : \mathbb{H}_{*}\left(\mathscr{E} / \Gamma ; \mathscr{S}_{*}(p)\right) \rightarrow \mathscr{S}_{*}(B \Gamma), \\
A_{\mathscr{C}^{\prime}} & : \mathbb{H}_{*}\left(\mathscr{E}^{\prime} / \Gamma ; \mathscr{S}_{*}\left(p^{\prime}\right)\right) \rightarrow \mathscr{S}_{*}(B \Gamma), \text { and } \\
A_{\mathscr{C}^{\prime}, \mathscr{E}}: & : \mathbb{H}_{*}\left(\mathscr{E}^{\prime} / \Gamma ; \mathscr{S}_{*}\left(p^{\prime}\right)\right) \rightarrow \mathbb{H}_{*}\left(\mathscr{E} / \Gamma ; \mathscr{S}_{*}(p)\right) .
\end{aligned}
$$

(We identify $B \Gamma$ with $E / \Gamma$.) The projections onto the second factors of $\mathscr{E} \times E$ and $\mathscr{E}^{\prime} \times E$ determine maps $q: \mathscr{E} \times_{\Gamma} E \rightarrow E / \Gamma$ and $q^{\prime}: \mathscr{E}^{\prime} \times_{\Gamma} E \rightarrow E / \Gamma$ respectively. These in turn determine $\bar{q} \in \operatorname{Mor}(p, *)$ and $\bar{q}^{\prime} \in \operatorname{Mor}\left(p^{\prime}, *\right)$ via the following commutative diagrams

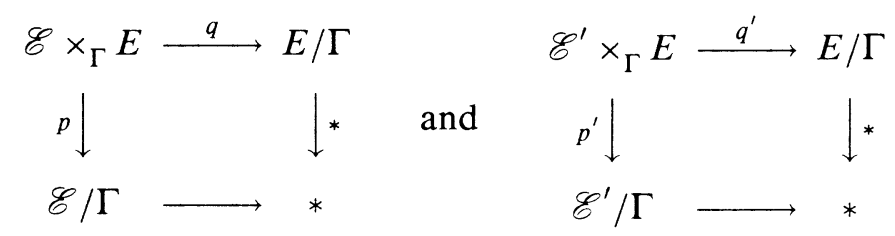

The assembly maps $A_{\mathscr{C}}$ and $A_{\mathscr{C}}$ are defined to be $\mathbb{H}_{*}(\bar{q})$ and $\mathbb{H}_{*}\left(\bar{q}^{\prime}\right)$ respectively. By Theorem A.2 there is a cellular $\Gamma$-map $\varphi: \mathscr{E}^{\prime} \rightarrow \mathscr{E}$ which is unique up to a cellular $\Gamma$-homotopy. It induces $\bar{\varphi} \in \operatorname{Mor}\left(p^{\prime}, p\right)$ by the commutative diagram

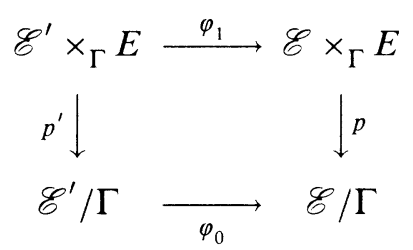

where $\varphi_{0}$ and $\varphi_{1}$ are induced by $\varphi$ and $\varphi \times$ id respectively. Then $A_{\mathscr{C}}, \mathscr{E}$ is defined to be $\mathbb{H}_{*}(\bar{\varphi})$. Since $\bar{q} \circ \bar{\varphi}=\bar{q}^{\prime}$, we have the following consequence.

A.9. Lemma. The assembly map $A_{\mathscr{C}^{\prime}}$ factors as the composite $A_{\mathscr{C}} \circ A_{\mathscr{C}}, \mathscr{C}$.

A.10. Theorem. The relative assembly map $A_{\mathscr{C}^{\prime}, \mathscr{C}}$ is an equivalence of $\Omega$ spectra, provided for each $S \in \mathscr{C}$ we have that $\dot{A}_{\mathscr{C}_{S}^{\prime}}$ is an equivalence of $\Omega$ spectra.

Proof of Theorem A.10. Recall that Proposition A.5 showed $\mathscr{E}^{\prime} \times \mathscr{E}$ equipped with the diagonal action of $\Gamma$ is a universal $\left(\Gamma, \mathscr{C}^{\prime}\right)$-space. Let $\hat{p}:\left(\mathscr{E}^{\prime} \times \mathscr{E}\right) \times_{\Gamma}$ $E \rightarrow \mathscr{E}^{\prime} \times_{\Gamma} \mathscr{E}$ denote the simplicially stratified fibration that it determines. The $\Gamma$-map $\varphi$ can be factored through $\mathscr{E}^{\prime} \times \mathscr{E}$ as the composite of $\Gamma$-maps id $\times \varphi: \mathscr{E}^{\prime} \rightarrow \mathscr{E}^{\prime} \times \mathscr{E}$ and $r: \mathscr{E}^{\prime} \times \mathscr{E} \rightarrow \mathscr{E}$, where $r$ denotes the projection onto the second factor. These in turn give a factorization of $\bar{\varphi}$ as $\bar{r} \circ \hat{\varphi}$, where $\hat{\varphi} \in \operatorname{Mor}\left(p^{\prime}, \hat{p}\right)$ and $\bar{r} \in \operatorname{Mor}(\hat{p}, p)$ are induced by $\mathrm{id} \times \varphi$ and by $r$ respectively. Hence $A_{\mathscr{C}^{\prime}, \mathscr{C}}=\mathbb{H}_{*}(\bar{\varphi})$ is the composite of $\mathbb{H}_{*}(\bar{r})$ with $\mathbb{H}_{*}(\hat{\varphi})$. But $\mathbb{H}_{*}(\hat{\varphi})$ is an equivalence of $\Omega$-spectra because of Theorem A.2 and the fact that both $\mathscr{E}^{\prime}$ and $\mathscr{E}^{\prime} \times \mathscr{E}$ are universal $\left(\Gamma, \mathscr{C}^{\prime}\right)$-spaces. This reduces the proof to showing that $\mathbb{H}_{*}(\bar{r})$ is an equivalence of $\Omega$-spectra. 
We now proceed as in the proof of Theorem A.8. Consider the commutative diagram where $r_{0}, r_{1}$ are the maps describing $\bar{r}$.

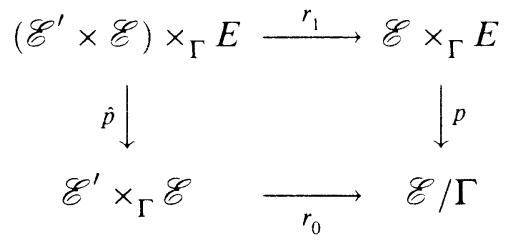

Filter $\mathscr{E} / \Gamma$ by subcomplexes $X_{m}$ such that $X_{m}-X_{m-1}$ is a single open cell $\sigma_{m}$ and $\bigcup_{m} X_{m}=\mathscr{E} / \Gamma$. (This is always possible when $\mathscr{E}$ is a countable complex. To handle the general situation, the following argument must be modified as indicated in Remark A.8.1.) Let $X_{m}^{\prime}=r_{0}^{-1}\left(X_{m}\right)$. These filtrations satisfy the condition of Remark A.7.1 for $r_{0}: \mathscr{E}^{\prime} \times_{\Gamma} \mathscr{E} \rightarrow \mathscr{E} / \Gamma$. To apply Remark A.7.1 it remains to verify Condition $(\mathrm{m})$ for each integer $m$; namely,

$$
\mathbb{H}_{*}(\bar{r}): \mathbb{H}_{*}\left(X_{m}^{\prime}, X_{m-1}^{\prime} ; \mathscr{S}_{*}(\hat{p})\right) \rightarrow \mathbb{H}_{*}\left(X_{m}, X_{m-1} ; \mathscr{S}_{*}(p)\right)
$$

is an equivalence of $\Omega$-spectra. When $\sigma_{m}$ is a 0 -cell $\mathbb{H}_{*}(\bar{r})$ can be identified with $A_{\mathscr{E}_{S}^{\prime}}$, where $S=\Gamma^{\sigma_{m}^{\prime}}$, the cell $\sigma_{m}^{\prime} \in \mathscr{E}$ is mapped to $\sigma_{m}$ under the quotient map $\mathscr{E} \rightarrow \mathscr{E} / \Gamma$, and $A_{\mathscr{C}_{S}^{\prime}}$ is an equivalence of $\Omega$-spectra by the hypothesis of Theorem A.10. More generally when $\sigma_{m}$ is an $n$-cell $\mathbb{H}_{*}(\bar{r})$ can still be identified with $A_{\mathscr{C}_{S}^{\prime}}$ (where $S=\Gamma^{\sigma_{m}^{\prime}}$ and $\sigma_{m}^{\prime}$ is a cell of $\mathscr{E}$ which is mapped to $\sigma_{m}$ under the quotient map $\left.\mathscr{E} \rightarrow \mathscr{E} / \Gamma\right)$ ) but with the spectra shifted by $n$. The hypothesis of Theorem A.10 again shows Condition $(\mathrm{m})$ is satisfied. Consequently Remark A.7.1 can be applied to complete the proof of Theorem A.10.

A.11. Remark. In this remark we use Theorem A.10 to complete the proof of the results discussed in 1.6.1, 1.6.2, and 1.6.4 above. First consider 1.6.1. Let $\mathscr{C}=\mathscr{G}(B \Gamma)$ and $\mathscr{C}^{\prime}=\{1\}$. Since $\Gamma$ is torsionfree, $\mathscr{C}$ consists of the trivial group together with every infinite cyclic subgroup of $\Gamma$. By results of Browder [5] and Quillen [46] it is known that $A_{\mathscr{C}_{S}^{\prime}}$ is an equivalence of $\Omega$-spectra for all $S \in \mathscr{C}$ provided either $\mathscr{S}_{*}()=\mathscr{L}_{*}()$ or $\mathscr{S}_{*}()=\mathscr{K}_{*}()$. Thus Theorem A. 10 together with Lemma A.9 show that conjecture (a) of 1.6 .1 is equivalent to Conjecture 1.6 for $\mathscr{S}_{*}()=\mathscr{K}_{*}(), \mathscr{L}_{*}^{-\infty}(), \mathscr{L}_{*}^{h}()$, or $\mathscr{L}_{*}^{s}()$.

Next consider 1.6.4. Let $\mathscr{C}=\mathscr{G}(B \Gamma)$ and $\mathscr{C}^{*}=\mathscr{G}^{\prime}(B \Gamma)$. The results of Cappell [7] and Yamasaki [57, 58] show that $A_{\mathscr{C}_{S}^{\prime}}$ is an equivalence of $\Omega$ spectra for all $S \in \mathscr{C}$ when $\mathscr{S}_{*}()=\mathscr{L}_{*}() \otimes \mathbf{Z}\left[\frac{1}{2}\right]$. We show in [23] the same result for $\mathscr{S}_{*}()=\mathscr{K}_{*}() \otimes \mathbf{Q}$. The argument in [23] uses a Frobenius induction technique (similar to that used in [12]) combined with [53] and [60]. Hence the implication mentioned in $\S 1.6 .4$ also follows from Theorem A.10 and Lemma A.9.

Finally we consider 1.6.2. Set $\mathscr{S}_{*}()=\mathscr{K}_{*}() \otimes \mathbf{Q}$ or $\mathscr{S}_{*}()=\mathscr{L}_{*}() \otimes$ Q. Let $\Gamma=\pi_{1} X, \mathscr{C}=\mathscr{G}(X), \mathscr{C}^{\prime}=\mathscr{G}^{\prime}(X)$, and $\mathscr{C}^{\prime \prime}=\{1\}$. We will now verify the assertion made in 1.6 .2 that the relative assembly map $A_{\mathscr{E}}^{\prime \prime}, \mathscr{E}$ 
induces a monomorphism on homotopy groups. Note that $A_{\mathscr{C}^{\prime \prime}, \mathscr{C}}$ factors as the composite $A_{\mathscr{C}^{\prime}, \mathscr{C}}{ }^{\circ} A_{\mathscr{C}}^{\prime \prime}, \mathscr{C}^{\prime}$. But it was shown in the last paragraph that $A_{\mathscr{E}}$, is an equivalence of $\Omega$-spectra. Consequently it suffices to show that $A_{\mathscr{C}^{\prime \prime}}, \mathscr{C}^{\prime}$ induces a monomorphism on the homotopy groups. Let $\mathscr{E}^{\prime}$ be a universal $\left(\Gamma, \mathscr{C}^{\prime}\right)$-space, and let $E^{\prime}$ be a universal $\Gamma$-space. Recall that $E=E^{\prime} \times \mathscr{E}^{\prime}$ equipped with the diagonal $\Gamma$-action is also a universal $\Gamma$-space by Proposition A.5. We may assume that $X=E / \Gamma$. Let $p: E \times_{\Gamma} E \rightarrow E / \Gamma, p^{\prime}: \mathscr{E}^{\prime} \times_{\Gamma} E \rightarrow$ $\mathscr{E}^{\prime} / \Gamma$, and $q: E / \Gamma \rightarrow \mathscr{E}^{\prime} / \Gamma$ denote the simplicially stratified fibrations induced by projection onto the two first factors and the second factor of $E \times E, \mathscr{E}^{\prime} \times E$, and $E^{\prime} \times \mathscr{E}^{\prime}$ respectively. Then $\alpha \in \operatorname{Mor}\left(p, p^{\prime}\right)$ and $\beta \in \operatorname{Mor}\left(p^{\prime}\right.$, id $)$ are described by the commutative squares in the following diagram:

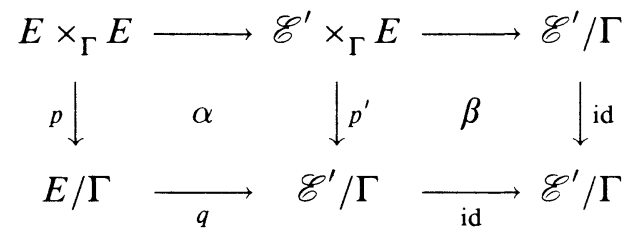

Since $A_{\mathscr{C}^{\prime \prime}, \mathscr{C}^{\prime}}=\mathbb{H}_{*}(\alpha)$, it will suffice by the functoriality of $\mathbb{H}_{*}($ ) to show that $\mathbb{H}_{*}(\beta \circ \alpha)$ is an equivalence of $\Omega$-spectra. But $\beta \circ \alpha=\delta \circ \gamma$, where $\gamma \in \operatorname{Mor}(p$, id) and $\delta \in \operatorname{Mor}(\mathrm{id}$, id) are given by the commutative squares in the following diagram:

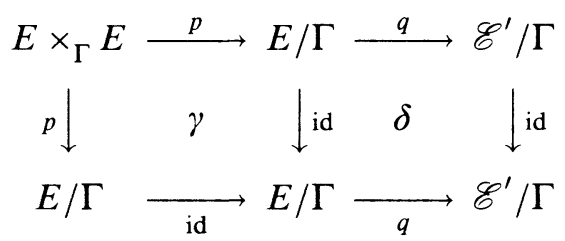

Hence $\mathbb{H}_{*}(\beta \circ \alpha)=\mathbb{H}_{*}(\delta) \circ \mathbb{H}_{*}(\gamma)$. Since $\mathbb{H}_{*}(\gamma)$ is an equivalence of $\Omega$-spectra by the homotopy axiom, it suffices to show that $\mathbb{H}_{*}(\delta)$ is also an equivalence of $\Omega$-spectra.

Note that $\mathbb{H}_{*}(\delta)$ is the map induced by $q$ in the homology theory for the spectrum $\mathscr{S}_{*}(\mathrm{pt})$. Since $\mathscr{S}_{*}(\mathrm{pt})$ is a product of Eilenberg-MacLane spectra, it will suffice to show that

$$
H_{*}(E / \Gamma, \mathbf{Q}) \rightarrow H_{*}\left(\mathscr{E}^{\prime} / \Gamma, \mathbf{Q}\right)
$$

is an isomorphism for ordinary homology theory with Q-coefficients. But this is a consequence of the Vietoris mapping theorem for simplicially stratified fibrations, since each fiber $q^{-1}(x), x \in \mathscr{E}^{\prime} / \Gamma$, is a $\mathbf{Q}$-homology point. To see this, note that $q^{-1}(x)=E^{\prime} / \Gamma^{y}$, where the point $y$ maps to $x$ under the quotient map $\mathscr{E}^{\prime} \rightarrow \mathscr{E}^{\prime} / \Gamma$. But $E^{\prime}$ is a universal $\Gamma^{y}$-space; i.e., $q^{-1}(x)=B \Gamma^{y}$.

This completes the verification of the claims made in 1.6.1, 1.6.2, and 1.6.4.

Remark A.12. In this remark we will prove Proposition 2.2. Let $\Gamma$ denote a (discrete group), and let $Y \rightarrow X$ denote a fibration over a connected CWcomplex $X$ having $\pi_{1} X=\Gamma$. Let $\widehat{Y} \rightarrow Y$ denote the pullback along $Y \rightarrow X$ of the universal covering projection $\widehat{X} \rightarrow X$; note that $\pi_{1} X$ acts on $\widehat{Y}$. We have the following generalization of Lemma A.9 and of Theorem A.10: 
A.12.1. If in the discussion proceeding the statements of Lemma A.9 and Theorem A.10 we replace the free action $\Gamma \times E \rightarrow E$ by the free action $\Gamma \times \widehat{Y} \rightarrow \widehat{Y}$, and we replace $B \Gamma$ by $Y$, then Lemma A.9 and Theorem A.10 remain true for the more general simplicially stratified fibrations

$$
\begin{aligned}
p: \mathscr{E} \times_{\Gamma} \widehat{Y} & \rightarrow \mathscr{E} / \Gamma, \\
p^{\prime}: \mathscr{E}^{\prime} \times_{\Gamma} \widehat{Y} & \rightarrow \mathscr{E}^{\prime} / \Gamma, \\
*: \widehat{Y} / \Gamma & \rightarrow *, \\
p_{S}^{\prime}: \mathscr{E}^{\prime} \times_{S} \widehat{Y} & \rightarrow \mathscr{E}^{\prime} / S, \quad S \in \mathscr{C},
\end{aligned}
$$

and associated assembly maps

$$
\begin{aligned}
A_{\mathscr{C}} & : \mathbb{H}_{*}\left(\mathscr{E} / \Gamma ; \mathscr{S}_{*}(p)\right) \rightarrow \mathscr{S}_{*}(Y), \\
A_{\mathscr{C}^{\prime}} & : \mathbb{H}_{*}\left(\mathscr{E}^{\prime} / \Gamma ; \mathscr{S}_{*}\left(p^{\prime}\right)\right) \rightarrow \mathscr{S}_{*}(Y), \\
A_{\mathscr{C}^{\prime}, \mathscr{C}}: & : \mathbb{H}_{*}\left(\mathscr{E}^{\prime} / \Gamma ; \mathscr{S}_{*}\left(p^{\prime}\right)\right) \rightarrow \mathbb{H}_{*}\left(\mathscr{E} / \Gamma ; \mathscr{S}_{*}(p)\right), \\
A_{\mathscr{C}_{S}^{\prime}} & : \mathbb{H}_{*}\left(\mathscr{E}^{\prime} / S ; \mathscr{S}_{*}\left(p_{S}^{\prime}\right)\right) \rightarrow \mathscr{S}_{*}(\widehat{Y} / S), \quad S \in \mathscr{C} .
\end{aligned}
$$

Moreover, given a universal $(\Gamma, \mathscr{C})$-space $\mathscr{E}$, the hypothesis of $\mathrm{A} .10$ can be weakened as follows: replace " $S \in \mathscr{C}$ " by " $S \in\left\{\Gamma^{x}: x \in \mathscr{E}\right\}$ ".

The proofs indicated for the original versions of Lemma A.9 and Theorem A.10 also work for these generalizations.

Now we can complete the proof of Proposition 2.2 as follows. Let $P: E \rightarrow X$ denote the fibration of 2.2 , and let $\xi=Y \rightarrow E$ denote a fibration over $E$ for which we wish to verify 1.7 ; note that the composite $Y \rightarrow E \rightarrow X$ is a fibration over $X$, which we denote simply by $Y \rightarrow X$. Set $\Gamma=\pi_{1} E$, and define two full classes of subgroups $\mathscr{C}^{\prime} \subset \mathscr{C}$ of $\Gamma$ by $\mathscr{C}^{\prime}=\mathscr{G}(E)$ and $\mathscr{C}=\left\{S: P_{*}(S) \in\right.$ $\mathscr{G}(X)\}$. Note that if $\pi_{1} X \times Z \rightarrow Z$ is a universal $\left(\pi_{1} X, \mathscr{G}(X)\right)$-space, then it is also a universal $(\Gamma, \mathscr{C})$-space, where $\Gamma$ acts on $Z$ through its quotient group $\pi_{1} X$. We can consequently identify the assembly map $A_{\mathscr{C}}$ (for the fibration $Y \rightarrow E$ ) with the assembly map $A_{\mathscr{G}(X)}$ (for the fibration $Y \rightarrow X$ ). In this way we see that $A_{\mathscr{C}}$ is an equivalence of $\Omega$-spectra since the first hypothesis of 2.2 yields that $A_{\mathscr{G}(X)}$ is an equivalence. But the second hypothesis of 2.2 implies that $A_{\mathscr{C}_{T}^{\prime}}$ is an equivalence of $\Omega$-spectra, where $T=P_{*}^{-1}(S)$ and $S \in$ $\mathscr{G}(X)$. Now it follows from A.12.1 applied to the fibration $\xi$, in which the universal $(\Gamma, \mathscr{C})$-space $\mathscr{E}$ is the above universal $\left(\pi_{1} X, \mathscr{G}(X)\right)$-space $Z$, that the assembly map $A_{\mathscr{C}^{\prime}}$ is also an equivalence. Since the assembly map for the simplicially stratified fibration $\rho(\xi): \mathscr{E}(\xi) \rightarrow \mathscr{B}(\xi)$ can be identified with $A_{\mathscr{E}}$, this completes the proof of Proposition 2.2.

\section{REFERENCES}

1. R. Anderson and W.-c. Hsiang, The functors $K_{-i}$ and pseudoisotopies of polyhedra, Ann. of Math. (2) 105 (1977), 201-223.

2. W. Ballmann, M. Gromov, and V. Schroeder, Manifolds of nonpositive curvature, Birkhäuser, Basel, 1985. 
3. H. Bass, Algebraic K-theory, W. A. Benjamin, New York, 1968.

3a. P. Baum and A. Connes, K-theory for discrete groups, Operator Algebras and Applications, Cambridge Univ. Press, London and New York, 1988, pp. 1-20.

3b. __ Chern character for discrete groups, A Fete of Topology, Academic Press, San Diego, CA, 1988, pp. 163-232.

4. M. Bokstedt, W.-c. Hsiang, and I. Madsen, Cyclotomic trace and the $K$-theoretic analogue of Novikov's conjecture, preprint.

5. W. Browder, Manifolds with $\pi_{1}=Z$, Bull Amer. Math. Soc. 72 (1966), 238-244.

6. D. Burghelea and R. Lashof, Stability of concordances and the suspension homomorphism, Ann. of Math. (2) 105 (1977), 449-472.

7. S. Cappell, Manifolds with fundamental group a generalized free product. I, Bull. Amer. Math. Soc. 80 (1974), 1193-1198.

8. D. Carter, Lower K-theory of finite groups, Comm. Algebra 8 (1980), 1927-1937.

9. F. Connolly and T. Koz' niewski, Finiteness properties of classifying spaces of proper $\Gamma$ actions, J. Pure Appl. Algebra, vol. 41, North-Holland, Amsterdam and New York, 1986, pp. 17-36.

10. G. F. Cooke and R. L. Finney, Homology of cell complexes, Math. Notes, vol. 4, Princeton Univ. Press, Princeton, NJ, 1967.

11. F. T. Farrell and W.-c. Hsiang, Manifolds with $\pi_{1}=G \times_{\alpha} T$, Amer. J. Math. 95 (1973), 813-848.

12. _ The Whitehead groups of poly-(finite by cyclic) groups, J. London Math. Soc. (2) 24 (1982), 308-324.

13. __ On Novikov's conjecture for cocompact discrete subgroups of a Lie group, Lecture Notes in Math., vol. 1051, Springer-Verlag, New York, 1984, pp. 38-48.

14. __ Addendum to: On Novikov's conjecture for cocompact subgroups of a Lie group, Topology Appl. 26 (1987), 93-95.

15. F. T. Farrell and L. E. Jones, Foliated control theory. I, K-theory 2 (1988), 357-399.

16. __ A topological analogue of Mostow's rigidity theorem, J. Amer. Math. Soc. 2 (1989), 257-370.

17. __ The surgery L-groups of poly-(finite or cyclic) groups, Invent. Math. 91 (1988), 559586.

18. K-theory and dynamics. I, II, Ann. of Math. (2) 124 (1986), 531-569; II, Ann. of Math. (2) 126 (1987), 451-493.

19. _ Algebraic $K$-theory of spaces stratified fibered over hyperbolic orbifolds, Proc. Nat. Acad. Sci. U.S.A. 83 (1986), 5364-5366.

20. Algebraic K-theory of discrete subgroups of Lie groups, Proc. Nat. Acad. Sci. U.S.A. 84 (1987), 3095-3096.

21. , Rigidity and other topological aspects of non-positively curved manifolds, Bull. Amer. Math. Soc. 22 (1990), 59-64.

22. Computations of stable pseudoisotopy spaces for aspherical manifolds, Algebraic topology, Poznan 1989, Lecture Notes in Math., vol. 1474, Springer, Berlin. Heidelberg, and New York, 1991, pp. 59-74.

23. __ Pseudoisotopy spaces of special affine manifolds, work in progress.

24. Stable pseudoisotopy spaces of non-positively curved manifolds, J. Differential Geom. 34 (1991), 769-834.

25. __ Topological rigidity for compact non-positively curved manifolds, Proc. Sympos. Pure Math., Amer. Math. Soc., Providence, RI (in press).

26. The lower algebraic $K$-theory of virtually $\infty$-cyclic groups, submitted for publication.

27. S. Gersten, On spectrum of algebraic K-theory. Bull. Amer. Math. Soc. 78 (1972), 216-219.

28. T. Goodwillie, unpublished. 
29. A. Hatcher, Concordance spaces, higher simple homotopy theory, and applications, Proc. Sympos. Pure Math., vol. 32, Amer. Math. Soc., Providence, RI, (1978) pp. 2-21.

30. W.-c. Hsiang, Geometric applications of algebraic $K$-theory, Proc. Internat. Congress Math. Warsaw, vol. 1 (1983), pp. 99-118.

31. B_ Borel's conjecture, Novikov's conjecture and the K-theoretic analogue, World Scientific book, Singapore.

32. B. Hu, Ph.D thesis, SUNY at Stony Brook, 1989.

33. __ A P _ geometric study of algebraic K-theory, Trans. Amer. Math. Soc. (to appear).

33a. __, Whitehead groups of finite polyhedra with non-positive curvature, J. Differential Geom. (to appear).

33b. _ unpublished.

34. I. M. James, Ex-Homotopy theory, Illinois J. Math. 15 (1971), 324-337.

35. L. E. Jones, The non-simply connected characteristic variety theorem, Proc. Sympos. Pure Math., vol. 32, Amer. Math. Soc., Providence, RI, 1978, pp. 131-140.

36. R. C. Kirby and L. C. Seibenmann, Foundational essays on topological manifolds, smoothings, and triangulations, Ann. of Math. Stud., vol. 88, Princeton Univ. Press, Princeton, NJ, 1977.

37. G. D. Mostow, Equivariant embeddings in Euclidean space, Ann. of Math. (2) 40 (1957), 432-446.

38. A. Nicas, On higher Whitehead groups of a Bieberbach group, Trans. Amer. Math. Soc. 287 (1985), 853-859.

39. __ Induction theorems for groups of homotopy manifold structures, Mem. Amer. Math. Soc. 267 (1982).

40. E. Pedersen and C. Weibel, A non-connective delooping of algebraic K-theory, Topology, Lecture Notes in Math., vol. 1126, Springer-Verlag, Berlin-Heidelberg-New York, 1985, pp. 166-181.

41. R. S. Palais, Imbedding of compact differentiable transformations groups in orthogonal representations, J. Math. and Mech. 6 (1957), 673-678.

42. M. S. Raghunathan, Discrete subgroups of Lie groups, Springer-Verlag, Berlin-HeidelbergNew York, 1972.

43. A. Ranicki, Algebraic L-theory II: Laurent extensions, Proc. London Math. Soc. 27 (1973), 126-158.

44. D. Sullivan, Lecture notes on the characteristic variety theorem, preprint.

45. A. Selberg, On discontinuous groups in higher dimensional symmetric spaces, International Colloquium on Function Theory, Tata Inst. of Fund Research, Bombay, 1960, pp. 147-164.

46. D. Quillen, Higher algebraic K-theory. I, Lecture Notes in Math., vol. 341, Springer-Verlag, Berlin-Heidelberg-New York, 1973, pp. 85-165.

47. F. Quinn, A geometric formulation of surgery, Topology of Manifolds, Proc. 1969 Georgia Topology Conf., Markham Press, Chicago, IL, 1970, pp. 500-511.

48. __ Ends of maps. II, Invent. Math. 68 (1982), 353-424.

49. __ Assembly maps in bordism type theories, preprint.

50. __ Algebraic K-theory of poly-(finite or cyclic) groups, Bull. Amer. Math. Soc. 12 (1985), 221-226.

51. Applications of topology with control, Proc. Internat. Congress of Math. (Berkeley, CA, 1986), Amer. Math. Soc., Providence, RI, 1987, pp. 598-606.

52. J. Wagoner, Delooping classifying spaces in algebraic K-theory, Topology 11 (1972), 349370.

53. F. Waldhausen, Algebraic K-theory of generalized free products, Ann. of Math. (2) 108 (1978), 135-256. 
54. _ Algebraic $K$-theory of topological spaces. I, Proc. Sympos. Pure Math., vol. 32, Amer. Math. Soc., Providence, RI, 1978, pp. 35-60.

55. C. T. C. Wall, Surgery on compact manifolds, Academic Press, New York, 1971.

56. J. A. Wolf, Spaces of constant curvature, Publish or Perish, Berkeley, CA, 1977.

57. M. Yamasaki, L-groups of crystallographic groups, Invent. Math. 88 (1987), 571-602.

58. __ L-groups of certain virtually solvable groups, Topology Appl. 33 (1989).

59. H. Zassenhaus, Über einen Algorithmus Bestimmung der Raumgruppen, Comment. Math. Helv. 21 (1948), 117-141.

60. C. A. Weibel, Mayer-Vietoris sequences and module structures on $N K_{*}$, Algebraic $K$ theory, Evanston 1980, Lecture Notes in Math., vol. 854, 1981.

Department of Mathematical Sciences, State University of New York, Binghamton, NEW YORK 13902

Department of Mathematics, State University of New York, Stony Brook, New York 11794 